\title{
Co-developing Openness: Indigenous Knowledge and Data Governance and Open Science in Canada
}

by

Amanda Hunter

A thesis submitted to the Faculty of Graduate and Postdoctoral Affairs in partial fulfillment of the requirements for the degree of

Master of Arts

in

Communication

Carleton University

Ottawa, Ontario

(c) (1) (2) (2)

2021, Amanda Hunter 


\begin{abstract}
Open science (OS) is a movement towards making the scientific process, and its outputs, more, transparent, accessible, inclusive, and credible, and OS has become a Government of Canada policy including the Canadian Commission for UNESCO. But how does open science impact Indigenous knowledge and data governance? Indigenous knowledge (IK) and Indigenous data and information challenge typical data and information norms in how they are collected, used, disseminated, and governed. As the Government of Canada is working to advance reconciliation and renew the relationship with Indigenous Peoples, there is an increased focus on institutionally recognizing and promoting the Indigenous right to knowledge and data governance. Although open science will likely increase the accessibility of scientific outputs, including research and knowledge published according to open standards, it is reasonable to assume that some of this research will include local and traditional knowledge and/or Indigenous data and therefore an OS framework should be developed in a way that prioritizes Indigenous knowledge and data governance.

In that light, in this master's thesis I aim to answer the following research question: How do the ideals of data, information, and knowledge sovereignty and governance, compare with those of open science, and is an Indigenous open science possible? I do so by conducting a literature review, a semi-structured interview with a First Nation Elder, and a comparative content analysis, and by analyzing the goals, objectives, standards, processes, and knowledge governance practices of both open science and Indigenous knowledge praxis. Here I consider the field of western science to have been shaped by colonial forms of empirical knowledge production and I argue that there are different world views, including Indigenous ways of knowing, that ought to be considered in the development of an OS framework to ensure a truly inclusive and mutually beneficial OS movement in Canada.
\end{abstract}




\section{Acknowledgements}

I would like to dedicate this work to the Algonquin Anishinaabe people whose unceded traditional land on which I completed my research. I acknowledge that the central themes of my research - including local and traditional knowledge, Indigenous science, and knowledge and data sovereignty - have been fundamental to First Nations, Métis, and Inuit peoples since long before I began to study them. I would also like to dedicate this research to my ancestors, without whom I would not be here today. Chi Miigwetch.

Writing a master's thesis during a global pandemic is the biggest accomplishment of my life and one that I could not have done without my incredible support system.

First and foremost, I would like to extend a huge thank you to Dr. Tracey P. Lauriault for your mentorship and supervision over the last two years. Thank you for your endless support and guidance, for nudging me in the right direction, and for helping me articulate my research goals when I was unable to articulate them coherently.

Thank you also to my committee whose steadfast guidance and input helped me to refine and clarify my research. To Dr. Peter Pulsifer and Dr. Shawn Graham: this project would not be what it is without your insight and feedback, and I am incredibly grateful for your time and interest. Also, Chi Miigwetch to Elder Gwen Phillips who graciously shared her wisdom and history with me. I hope this research makes the impact we envisioned.

Finally, to my friends and family whose endless support reminded me that I could, in fact, finish this project: I love and appreciate you endlessly. Mom, Dad, Brandon, Zac, and Mack: thank you for constantly reminding me that I am capable and smart, if not a tad dramatic. A big thank you to my COMM-rades who checked in when we could not get together in person

and assured me that I was not alone in feeling hopelessly behind. Our work sessions and your constant encouragement truly carried me throughout this process, and I am tremendously grateful to know you all. 


\section{Table of Contents}

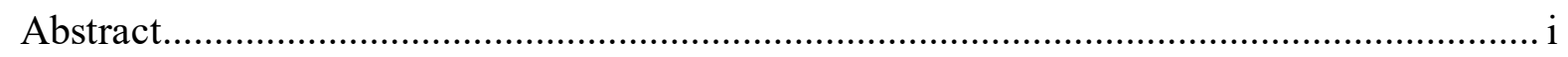

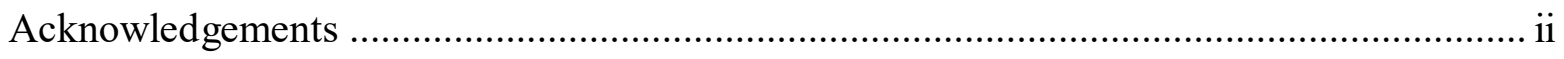

Table of Contents..................................................................................................... ii

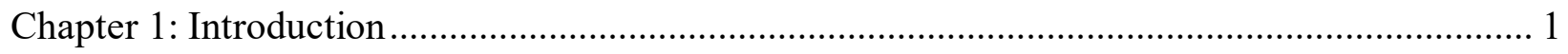

1.1. Inclusive Data Representations: Project Inspiration........................................... 1

1.2. Significance of the Research and the Research Question...................................... 3

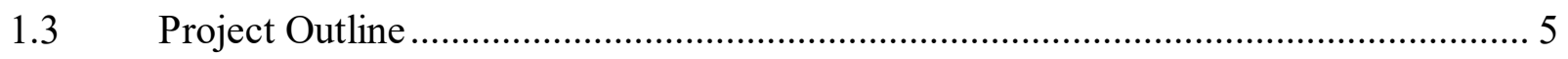

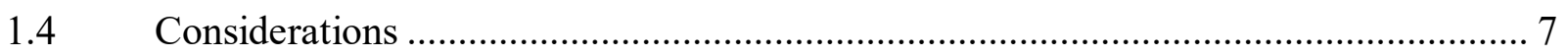

Chapter 2: Theoretical Framework \& Key Concepts ....................................................... 9

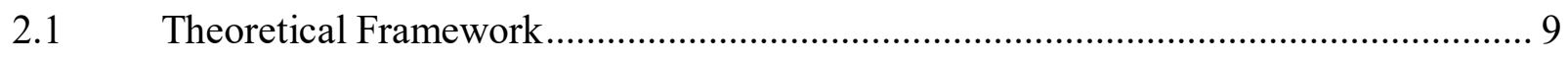

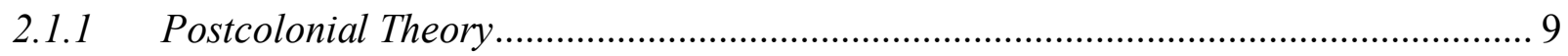

2.1.2 Social Construction of Scientific Knowledge ...................................................... 11

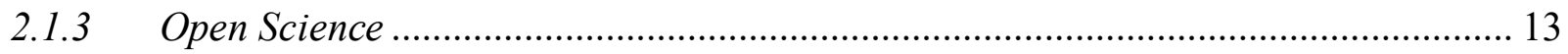

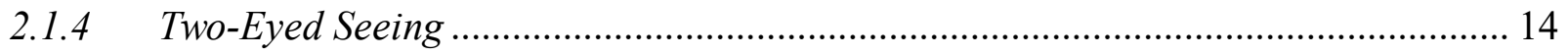

2.1.5 Critical Race Theory and Tribal Critical Race Theory ......................................... 15

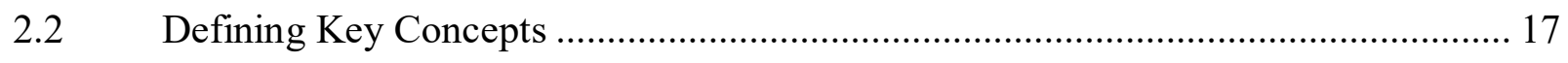

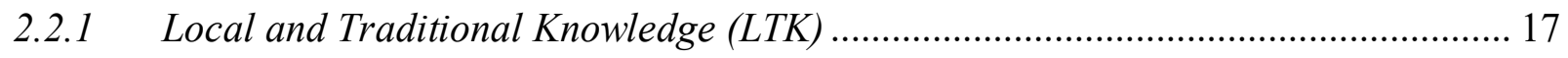

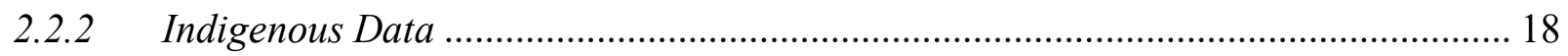

2.2.3 Indigenous Knowledge and Data Governance .................................................. 20

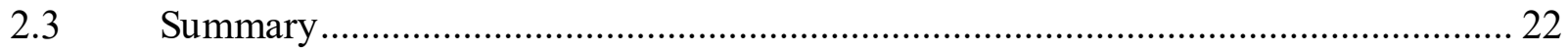

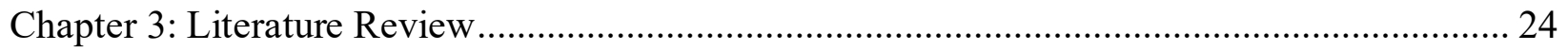


3.1 Decentralizing Western Knowledge, Decolonizing Science \& Indigenous Knowledge 25

3.2 Contextualizing and Considering Open Science ..................................................... 31

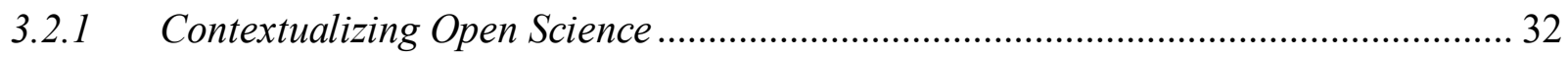

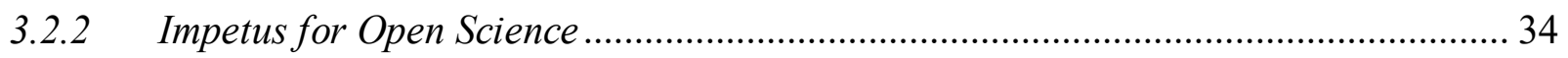

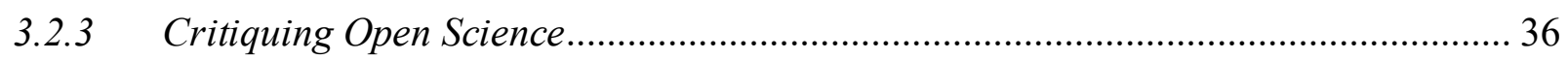

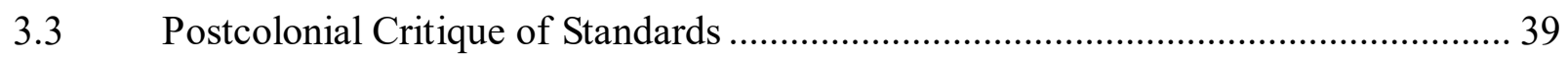

3.4 The State of Co-Development Research in Canada ............................................................. 44

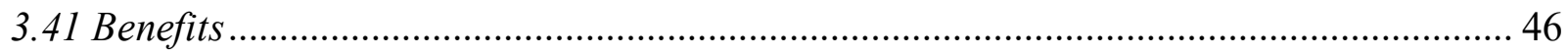

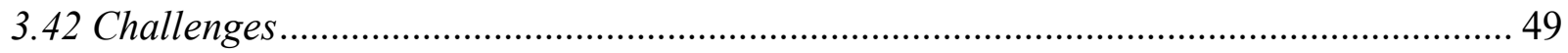

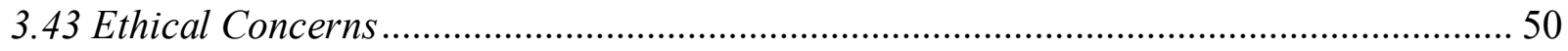

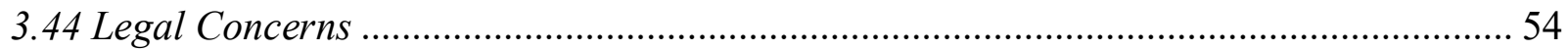

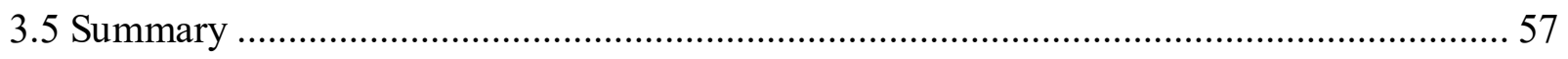

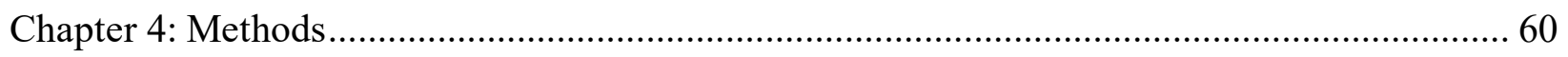

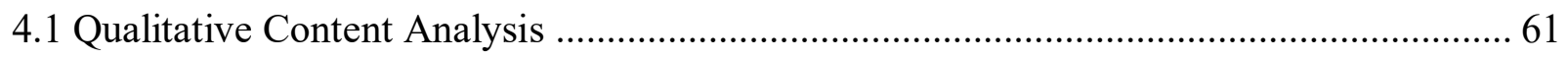

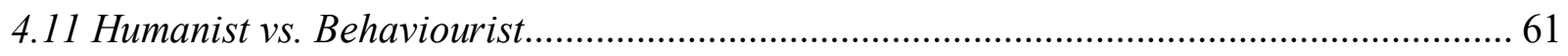

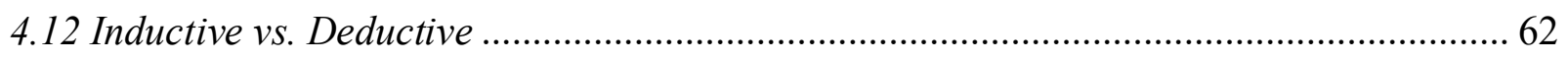

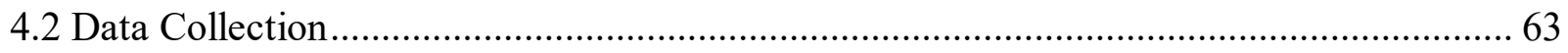

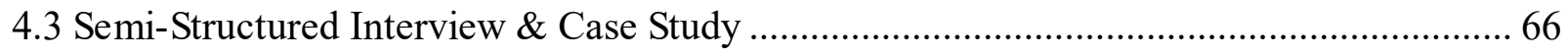

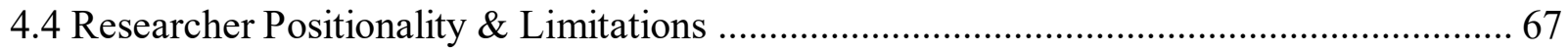

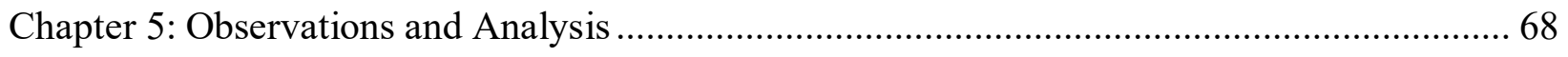

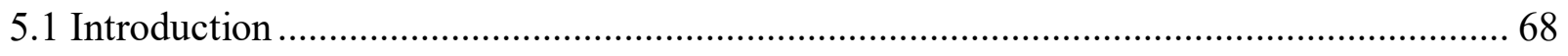

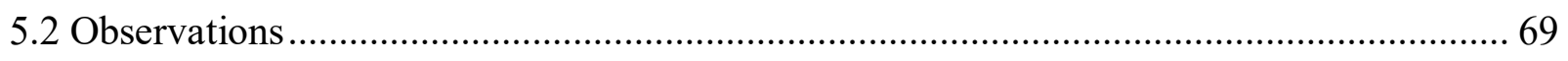

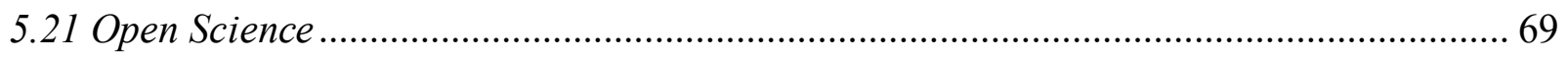

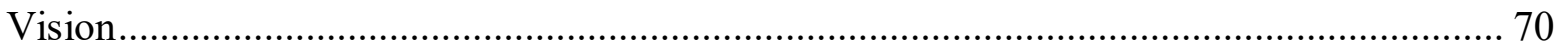




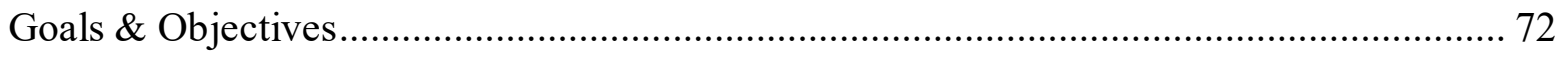

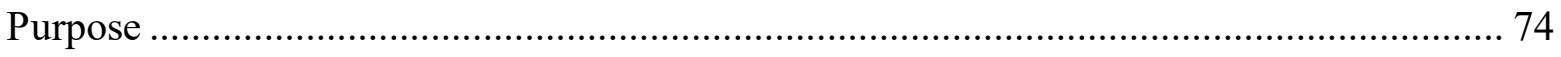

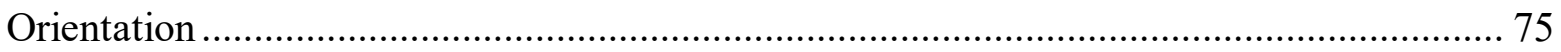

Philosophical Investigation................................................................................................ 77

5.22 Indigenous Knowledge and Data Governance................................................................ 77

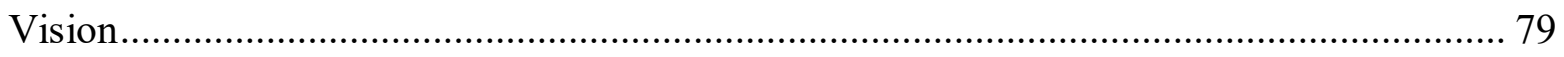

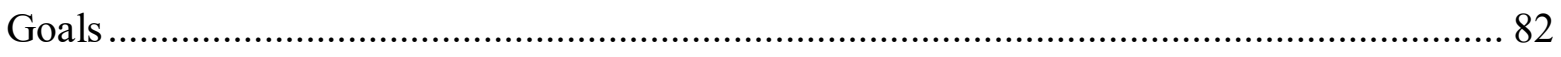

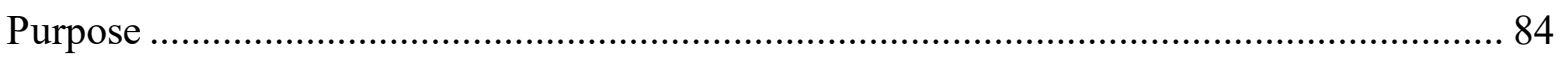

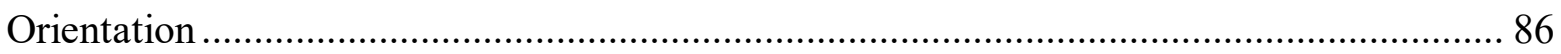

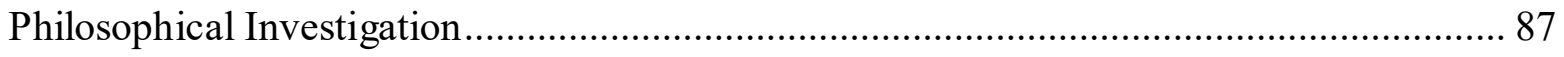

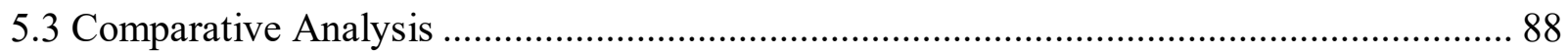

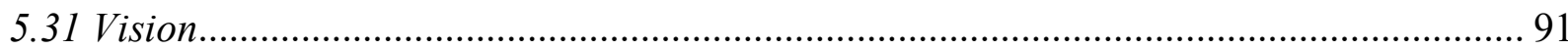

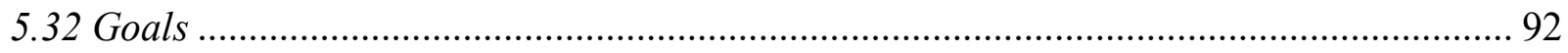

5.33 Purpose

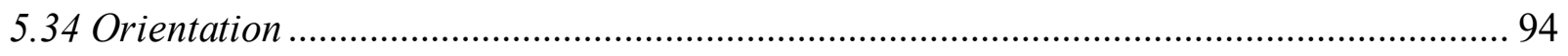

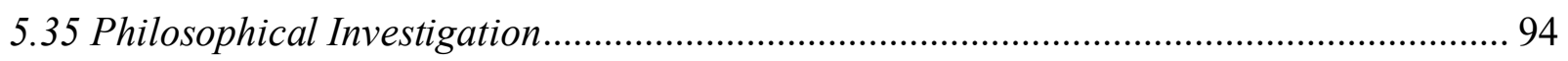

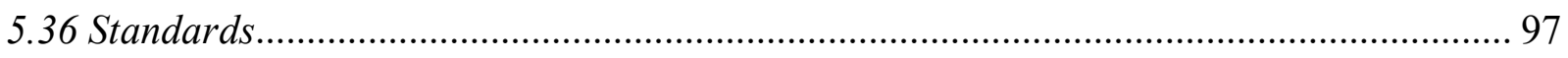

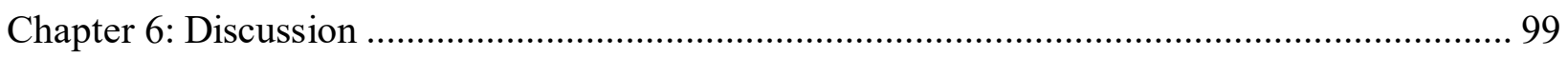

6.1 Back to the Research Questions …………………….................................................... 100

6.2 Semi-Structured Interview: The Impact of Data Governance......................................... 101

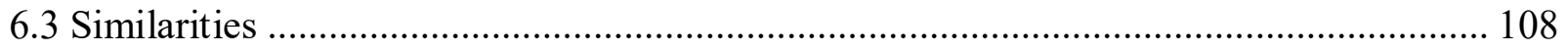

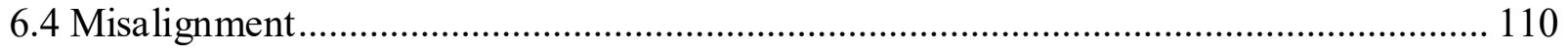

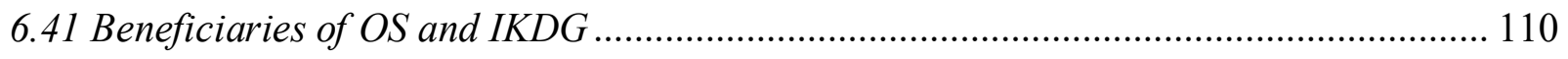

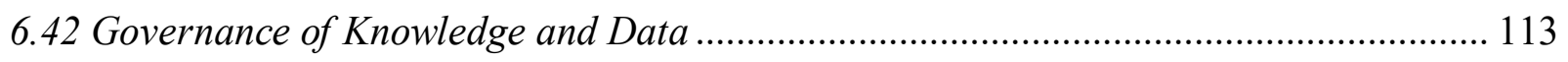




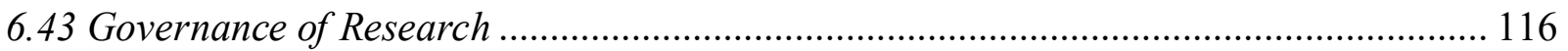

6.5 Broader Implications of Findings .............................................................................. 119

6.51 Techno-Solutionism and Techno-Utopianism ............................................................... 120

6.52 Producing and Reinforcing Western Knowledge ………............................................. 124

Chapter 7: Recommendations, Future Research, and Conclusion ............................................. 128

7.1 Research Summary .................................................................................................. 131

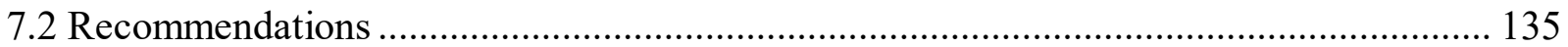

Recommendation 1 - Indigenous knowledge and data governance should be included, by

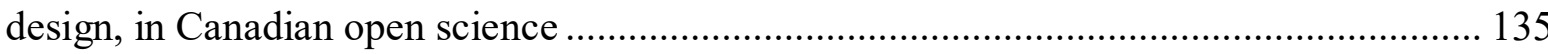

Recommendation 2 - Increased focus on infrastructural barriers to Indigenous knowledge

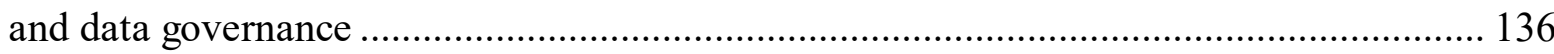

Recommendation 3 - Develop a strategy, database, or standard to support Indigenous knowledge and data governance in scientific research with metadata tags for Indigenous knowledge and data …….................................................................................................. 137

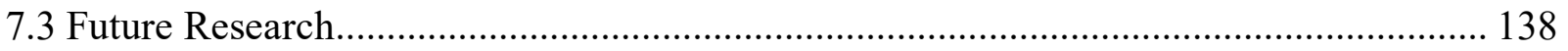

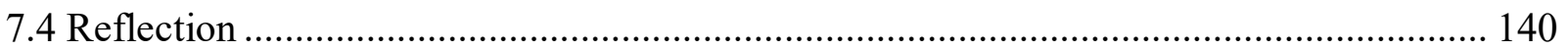

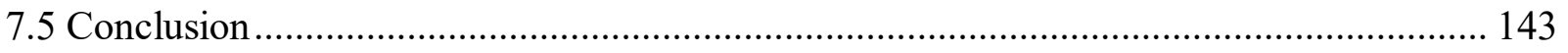

Appendix 1. Ethics Application Package................................................................................. 145

1A. Research Ethics Protocol Form ………………........................................................... 145

1B. TCPS2 Core Certification......................................................................................... 152

Appendix 2. Semi-Structured Interview Description and Materials ........................................... 153

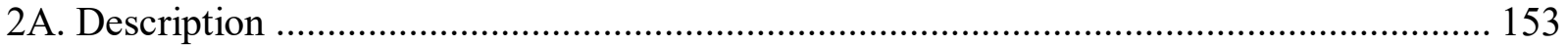

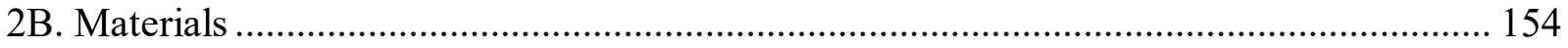

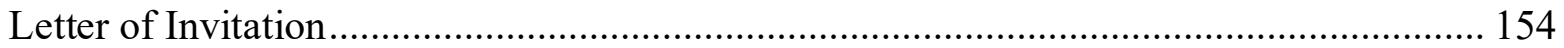

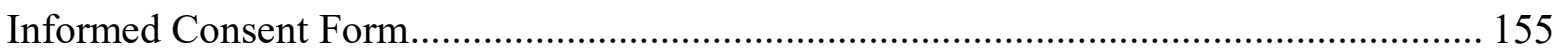


References.

\section{List of Tables}

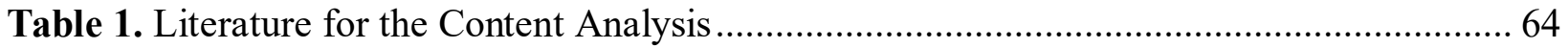

Table 2. Open Science vs. Indigenous Knowledge and Data Governance Summary ................... 88

Table 3. Open Science vs. Indigenous Knowledge and Data Governance: Commonalities \&

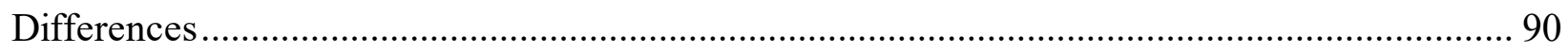

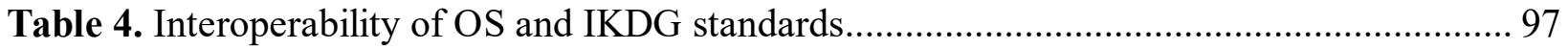




\section{Chapter 1: Introduction}

\subsection{Inclusive Data Representations: Project Inspiration}

The inspiration for this thesis results from my observations and experience of the evolution of data collection and management during the novel coronavirus (COVID-19) pandemic. During the constantly evolving global emergency, access to accurate qualitative and quantitative data has been key to enhancing the collective understanding of COVID-19 and its genetic structure, rate of infection, and symptom profile. This rapid sharing of data and information has been made possible largely due to open science (OS) policies which removed barriers to the free flow of research, allowing researchers across the globe to collaborate, access information more rapidly, and expand on existing bodies of work in genetics, epidemiology, and public health to name a few. In May 2020, the Organization for Economic Co-operation and Development (OECD) declared that open science is critical to combatting COVID-19, as OS policies and practices have accelerated the pace of research and the global dissemination of the outputs of science (OECD, 2020).

The pandemic revealed a conundrum regarding data collection and open data sharing. In the early months of the pandemic, it became evident that COVID-19 disproportionately affected 
sociodemographic groups of people differently, and that identity-based data for Indigenous, Black, and racialized minority individuals was noticeably lacking (Statistics Canada, 2020). The pandemic began to intensify pre-existing inequities, but without appropriate data to substantiate and rationalize their experiences, these communities faced more dangerous consequences including increased mortality rates and inadequate access to medical care globally. (Al Saba, 2020). As calls for the intensified collection of more granular and disaggregated data increased, health officials struggled to locate responsible practices of data sharing. While a lack of appropriate and relevant data can result in the invisibility of diverse circumstances, concerns remain over the potential for these data to perpetuate discrimination against racialized communities when shared publicly (Statistics Canada, 2020).

This example illustrates a potential issue that arises regarding open science and open data sharing in Canada. While data are critical to the collective understanding of a phenomenon, what remains unclear is appropriate degrees of data sharing in various contexts, with different forms of knowledge and data pertaining to diverse cultures. As an Ojibwe woman and critical data studies scholar, this puzzled me in a unique way. I am inspired by the capacity for data and knowledge sharing to empower Indigenous peoples, though I am mindful of contextual appropriateness, and the notion that not all data and knowledge should be treated the same. For instance, can open science standards function to protect the knowledge that is sacred to local and traditional cultures when it is applied in research? What about data produced by - and for Indigenous communities? How can the two knowledge systems be co-managed for mutual benefit? These questions inspired me to pursue this project. The objective of this Master thesis is to therefore interrogate the compatibility of open science practices with Indigenous data and knowledge governance and determine whether Indigenous open science is possible. In the 
context of my research, the term 'Indigenous' refers to the three distinct Aboriginal groups in Canada - First Nations, Métis, and Inuit.

\subsection{Significance of the Research and the Research Question}

This study is relevant and timely as Canada is at the intersection of two important movements. The first is reconciliation, which has led to an increased recognition of the Indigenous right to self-determination and self-governance, including recognizing traditional knowledge and ways of knowing becoming mainstream in research. The second movement is the Government of Canada's mandated adoption of open science (OS). Open science was formally adopted as policy by the Government of Canada, the Canadian Commission for UNESCO (a branch of the United Nations Educational, Scientific and Cultural Organization), and research institutions, and is increasingly being encouraged by granting councils such as the Natural Sciences and Engineering Research Council of Canada (NSERC), the Social Sciences and Humanities Research Council of Canada (SSHRC), and the Canadian Institute for Health Research (CIHR) (Government of Canada, 2016). This means that there is an increased focus on policies and mandates from the Government of Canada to respect Indigenous self-determination and sovereignty (including data sovereignty), and a movement towards making scientific processes, including data sharing, more open and accessible. Consequently, here I suggest, it is necessary to analyze the ways in which these two practices interact, align, and conflict, as they each have different epistemologies, standards, methods, and goals.

For example, the goals of open science include increased transparency, credibility, replicability, and accessibility of scientific research, as open science considers scientific knowledge to be a public good. The goals of Indigenous knowledge and data governance 
(IKDG), however, are to protect and preserve traditional knowledge, and empower Indigenous individuals to practice governance over their own data. This is especially important since Indigenous peoples have historically been research subjects instead of consenting participants (Drawson et al., 2017; Hyett et al., 2019; FNIGC, 2020) and their knowledge and data have been exploited, shared, and reused without adequate consent, and manipulated to uphold stereotypical and deficit conceptions of Indigenous peoples (Hyett et al., 2019; FNIGC, 2020). Traditional knowledge is also not afforded the same protections as Western knowledge in terms of intellectual property and copyright, so there is a well-documented history of exploitation of traditional knowledge (TK) which should be avoided in the future (Anderson, 2015; FNIGC, 2020). Conceptually, it might appear that the process of open science - making research and data open by default and accessible to as many people as possible - could work to further enable this exploitation. However, this does not necessarily mean that they are incongruous, as open sharing within communities could benefit Indigenous peoples, and traditional knowledge/science in the mainstream domain could enable the decolonization of Indigenous knowledge.

This example demonstrates the need to further explore the goals and processes of Indigenous knowledge and data governance, and the processes and standards of open science, to determine how they might align. Open science is still a relatively new concept, and the rules, standards, policies, and methods which shape the process have not yet normalized as a practice (Hampson et al., 2020). Also, the confluence of the newness of open science, and of First Nations, Métis, and Inuit knowledge governance in Canada, may be attributed to the sparse amount of literature that examines the intersection of these concepts. The literature that I encountered largely focuses on the implications of open science for the Global South in comparison to the Global North. This focus on North versus South has resulted in a knowledge 
gap, as the consideration of the 'Global North' as a singular experience does not address the specific perspectives of First Nations, Métis, and Inuit communities and researchers in the open science discussion. This thesis aims to provide insight about this gap by comparing the concepts of open science and Indigenous knowledge and data governance, considering their historical roots and contexts, primary goals, and methods of application and standardization.

My primary research question is: How do the ideals of data, information, and knowledge sovereignty and governance, compare with those of open science, and is an Indigenous open science possible?

\subsection{Project Outline}

I aim to answer this question by applying a three-step methodological approach including: a literature review, semi-structured interview and a comparative content analysis of the literature supporting OS and Indigenous knowledge and data governance in Canada. The remainder of this research will be organized in the following way: In Chapter 2 I will present the theoretical framework and key concepts which inform my research. I will describe my hybrid theoretical framework which draws on the knowledge of postcolonial theory, the social construction of scientific knowledge, theories of open science, two-eyed seeing, and critical race theory. I will explore the concepts of local and traditional knowledge (LTK), Indigenous data and information, and knowledge and data governance. Chapter 3 will explore the relevant literature to identify how dominant knowledge is produced in the postcolonial context, explore common benefits and challenges in open science, examine post-colonial critiques of standards, and the current state of co-development in Canadian scientific research. In Chapter 4, I will describe data collection method involving a mixed methods approach that includes a comparative 
content analysis and case study developed from a semi-structured interview with an expert professional in data governance, Elder Gwen Phillips. In Chapter 5 I present the results of the content analysis to demonstrate the vision, goals, purpose, orientation, and philosophical underpinnings of open science and Indigenous knowledge and data governance. The comparative analysis will determine where the concepts and goals of open science align with those of Indigenous knowledge within the theoretical frameworks and concepts just listed. I will also analyze the goals and priorities of Indigenous knowledge and data governance (determined from the interview) and provide possible conclusions about its importance and impact considering the interview. The documents I selected for the content analysis include directives from the Government of Canada towards the implementation of an open science framework including the Roadmap for Open Science, the 2018-2020 National Action Plan on Open Government, and the Open by default and modern, easy to use formats. These were selected because these are the key mandates guiding OS implementation by the Government of Canada and set the parameters for its adoption. I also assessed key principles such as The CARE Principles for Indigenous Data Governance and The FAIR Guiding Principles for scientific data management and stewardship as they are important practices that define and contextualize the standards for data management applied in the context of open data, open science, and Indigenous knowledge and data governance. I also examine a policy paper by the OECD for Making Open Science a Reality as it is the most comprehensive document describing the purpose, methods, and goals of open science on a global scale. Finally, I analyzed the following Indigenous knowledge and data governance resources: The Principles of Ethical Métis Research prepared by the National Aboriginal History Organization, Inuit Tapiirit Kanatami's National Inuit Strategy on Research, the Ownership, Control, Access and Possession (OCAPTM): The Path to First Nations Information Governance 
by the First Nation Information Governance Centre, and the MUKURTU digital archive, developed by Warumungu community members to empower Indigenous communities in their endeavor to preserve and share traditional knowledge in an ethically conscious, culturally specific, and sustainable way. In Chapter 6 I discuss my findings, incorporating the literature review from Chapter 3 to critically analyze the congruence of open science and Indigenous knowledge and data governance and determine whether these align in theory, conceptually, and in practice. In Chapter 7 I will draw conclusions ascertained from the methodology, make recommendations based on my findings, and discuss potential directions for future research in this domain.

\subsection{Considerations}

It is important to make three major clarifications. Firstly, the primary objective of this work is to provide insight to Indigenous communities and organizations who are unsure of the potential implications of open science for their governance of knowledge, data, and information. This work attempts to determine what aspects of the goals, standards, and practices of open science could benefit existing conceptions and practices of data sovereignty and governance; it will also consider how the system of open science aligns with the existing system of traditional knowledge and Indigenous data governance, including the related processes of production, preservation, dissemination, and interpretation. My research does not ask 'does traditional knowledge fit with the model of open science?'; instead, it prioritizes and respects the traditions of Indigenous praxis and governance, asking 'how might the open science framework also benefit Indigenous peoples and respect the goals of data governance and sovereignty?'. 
Second, this work does not attempt to speak on behalf of any group and does not assert that the opinions and/or priorities included by various organizations speak for any collective group of Indigenous peoples. I recognize that there are hundreds of different communities of First Nations, Metis, and Inuit peoples whose cultures, traditions, and local and traditional knowledge are distinct from one another. My goal is not to homogenize the distinct Aboriginal groups - and the various cultures and communities within these larger groups - whose land on which I conduct this research. My research aims to provide an analysis of the open science ecosystem - including epistemological roots, practical goals, and methodological processes, including standards - to determine what could be benefitted from its adoption by Indigenous peoples, and locate potential tensions. If utilized for future research, this project should be treated as a loose framework through which to consider how diverse forms of Indigenous knowledge can be articulated in the open science model. It is not meant to be a definitive consensus or statement on the open science movement on behalf of Indigenous peoples, nor does it suggest that 'Indigenous knowledge and data governance' is a singular, standardized, homogenous practice.

Finally, it is important to acknowledge the ongoing development of the open science concept. Globally, there is currently no single agency directing the coordination of the open science movement (Hampson et al., 2020): therefore, there is no definitive open science praxis, no concrete, set-in-stone standards, and no single set of best practices. The movement toward open science is still very much in development, which is why a renewed dialogue about the (mis)alignment of OS with other ways of knowing is critical. However, this research can only speak to the current iteration of open science as described in the following chapter. 


\section{Chapter 2: Theoretical Framework \& Key Concepts}

This chapter begins by describing the theoretical framework which grounds my research. Here I outline my hybrid theoretical framework which draws on postcolonial theory, social construction of scientific knowledge, open science, two-eyed seeing, and critical race theory to inform my expectations and assumptions about the convergence of Western and Indigenous knowledge systems. In section 2.2 I define key concepts and distinguish between local and traditional knowledge (LTK) and Indigenous data and information, before describing the historical context of local and traditional knowledge governance.

\subsection{Theoretical Framework}

\subsubsection{Postcolonial Theory}

Postcolonial theory is a school of thought primarily focused on understanding the various global impacts of European colonial imposition between the 18th and 20th century (Elam, 2019) and its influence on identity, language, representation, and history. Postcolonial theory posits that the world as we know is impossible to understand without the historical context of imperialism 
and colonial rule (Elam, 2019), and the central goal of this work is postcolonial reclamation. The founding of postcolonial theory is credited to Edward Said who coined the term 'orientalism' in his 1978 book by the same name. As the theory entered mainstream academic discourse in the 1980s in the US and UK as "part of larger wave of new and politicized fields of humanistic inquiry” (Elam, 2019, para. 1), scholars (Bhabha, 1994 \& 1996; Ashcroft et al., 1989;

Spivak, 1990) continued to build on his work. Postcolonial theory appears to have emerged in the Canadian Indigenous discourse in the 1990's with postcolonial scholar Laura Moss's paper, “Is Canada Postcolonial?" (Moss, 1999, p. v).

Post-colonial theory is important here because it is primarily focused on the theories, goals, objectives, and practices of Indigenous knowledge and data governance in Canada. Postcolonial theory is the practice of "theoretical resistance to the mystifying amnesia of the colonial aftermath" (Gandhi, 1998, p. 4) which is performed by way of "academic revisiting, remembering, and interrogating the colonial past" (ibid). This approach is essential to contextualizing the current state of Indigenous knowledge and data governance, as the impetus for contemporary Indigenous governance practices - including self-determination, sovereignty, and even governmental reconciliation - are predicated on legacies of colonialism in Canada. Furthermore, the rationale for understanding the nuanced handling, preserving, and sharing of Indigenous knowledge and data ought to be contextually situated in data colonialism and knowledge exploitation, specifically in the Canadian context. Data colonialism is a form of $21 \mathrm{st}$ century colonialism which "combines the predatory extractive practices of historical colonialism with the abstract quantification methods of computing" (Couldry \& Mejias, 2018, p. 337). Postcolonial theory therefore informs my analysis of open science and the potential to align with knowledge governance praxis. Postcolonial theory is useful to better understand the inequities 
that exist with respect to the prioritization of Western knowledge in mainstream domains and institutional foundations including public policy, healthcare, education, and science, making conclusions about whether open science is postcolonial.

\subsubsection{Social Construction of Scientific Knowledge}

The theory of natural science as socially constructed seeks to destabilize the view of hard science as natural, unbiased, and objective fact. Dr. Md. Abdul Mannan, a scholar whose work is focused on the intersection of science and philosophy, explains that science and scientific knowledge are generally considered to be "free from any kind of human attitude and strictly based on observations, experiments, [and] logical analyses of its concepts" (Mannan, 2016, p. 44) which aims to provide an objective description of the real world (ibid). Conceptualizing scientific knowledge as socially constructed, however, destabilizes this common view of the natural sciences as being about objective truth as there is an acknowledgement of the researcher's positionality within the production of scientific knowledge, and more widely, the social environments in which knowledge is produced. In this view, science cannot be completely objective since scientists are humans who exist as social beings, therefore:

No scientist is beyond his psychology, ideology and sociology which have significant impact on his thought. All these factors produce influences over scientific decisions, such as decisions about when a phenomenon is recognized as a problem, what the methods are for solving the problem, what the methods are for justification, how different theories are to be compared, when the research comes to end, etc. This line of investigation will clearly show that scientific knowledge possesses one kind of subtle subjectivity in its nature (Mannan, 2016, p. 44).

The theory is, in part, rooted in the philosophical theory of relativism (Akena, 2012), and shaped by several relativist scholars. Karl Manheim (1936), an influential German sociologist, believed knowledge to be a social phenomenon which cannot be understood outside of the social 
circumstances in which it is constructed. Psychologist and scholar Herbert Sprott (1954) suggested that knowledge was socially determined to serve a particular purpose in society. Philosopher and sociologist Gerard De Gre (1941) argued that truth is relative to the society in which it is constructed. It is beyond scope here to delve into all the particularities of relativism, and suffice it to say, that here I apply this thinking to understand the relational nature of knowledge and knowledge production. Relativism at its most basic is the understanding that "a phenomenon $x$ (e.g., values, epistemic, aesthetic and ethical norms, experiences, judgments, and even the world) is somehow dependent on and co-varies with some underlying, independent variable $y$ (e.g., paradigms, cultures, conceptual schemes, belief systems, language)" (Baghramian \& Carter, 2015). I therefore in this thesis conceptualize science as more relative than objective, in that it is most certainly dependent on, or co-varies with, the underlying cultures, societies, belief systems, and social environments in which it is produced (Mannan, 2016).

Those involved in the emerging field of critical data studies also consider knowledge to be socially constructed and suggest that data, as the building blocks of knowledge, are not neutral and that data and knowledge exist within a larger socio-technological assemblage that influences the modes of data collection within various contexts (Kitchin, 2014; Lauriault, 2019). By extending these understandings of science, I accept that scientific data and knowledge cannot exist "independently of the ideas, instruments, practices, contexts and knowledges used to generate, process and analyze them" (Kitchin, 2014, p. 1). Thus, it is understood that science, like all knowledge production endeavors, are social constructions; and consequently, scientific methods in Canada, unless otherwise stated, are reflective of Western values, beliefs, and histories, which influence scientific output. Understanding scientific 'facts' as contextual and 
socially constructed is important here since I acknowledge that Indigenous knowledge, in all of its manifestations, is an equal form of culturally, community and contextually situated scientific knowledge and that open science may need to change as a result since it may be possible that the governance of IK data and information can benefit from OS.

\subsubsection{Open Science}

Open Science (OS) is a movement and practice which seeks to address issues of reproducibility, credibility, transparency, and accessibility of modern scientific methods. Open science (OS) does not have a single fixed definition; it is operationalized in several ways and functions within a larger taxonomy that includes open access, open publishing, open data, open source, and open research. Despite its various iterations, all definitions of open science (OS) are underscored by the goal of opening the scientific process and making its outputs accessible on a wider scale. This is because the 'theory' of open science posits that "there currently exists a gap between scientific values and scientific practices" (Spies, 2013, para. 1): primarily that science should be more transparent, collaborative, accessible, and democratic than it currently is. OS is closely related to open access $(\mathrm{OA})$ in this way. OA refers to the process and policies through which research outputs are made available in online formats that are free and easily accessible. OS might include OA, especially in the way that scientific research outputs are disseminated and made openly available, however OS refers to the entire opening of the scientific process from research design, processes, technologies, standards, methodologies, source code, data, and OA publishing, and of course depositing the data, thus the full data lifecycle. The aim of open science is to transform the scientific process from conception to publication, enabling a more collaborative scientific process, and expanding the range of actors so that even citizens can participate in the production of scientific knowledge (FOSTER, n.d.; Chan, 2019). 
The benefits of adopting these open science methods are well-documented, and it is generally hoped that open science is the future of scientific practice. For instance, as I will address in chapter 3 , it has been suggested that OS can provide common resolution to issues such as the inaccessibility of research outputs (Apesi \& Brand, 2020), irreproducibility in modern science (Shapin, 2015), and address the marginalization of non-Western researchers in Westerndominated fields (Allen \& Mehler, 2019; LeBel, Campbell \& Loving 2017). It is generally considered a more democratic way of producing knowledge, as it allows citizens to collaborate in the agenda-setting and policy development processes (Chan, 2019). In theory, this should allow for a more diverse range of perspectives in a traditionally exclusive practice. This is important to this research as I will critically analyze open science and the praxis of Indigenous knowledge and data governance to determine how well OS might account for diverse forms of knowledge.

\subsubsection{Two-Eyed Seeing}

This research is grounded in the traditional concept of two-eyed seeing, or Etuaptmumk, which refers to the practice of drawing from "two existing knowledge systems...[which] encourages equality, mutual respect, support, and cooperation” (Snively \& Williams, 2018, p. 5). Mi'kmaq elder Albert Marshall coined the phrase which draws on the guiding principles of Mi'kmaq knowledge to recognize the benefit of employing two or more perspectives and achieve more meaningful outcomes. Marshall (2017) defines two-eyed seeing as "Learn[ing] to see from your one eye with the best or the strengths in the Indigenous knowledges and ways of knowing ... and learn to see from your other eye with the best or the strengths in the mainstream (Western or Eurocentric) knowledges and ways of knowing ... but most importantly, learn to see with both these eyes together, for the benefit of all” (Marshall, 2018, para. 3). Western and traditional 
methods of science have been historically viewed in opposition (Little Bear, 1993; Berkes, 1994; Little Bear, 2009; Magga, 2009). However, two-eyed seeing, also called co-development, is about collaboration: seeing the value in articulating two differing knowledge frameworks alongside one another rather than considering them in opposition. This concept will guide this research which will help to distinguish the foundational and epistemological differences between the two methods and will analyze the ways in which they may or may not perfectly align. However, the goal of this thesis is to identify the ways in which they can (and do) align, and to recognize the benefits that open science can provide for the continued co-development of Indigenous and Western science. It will not juxtapose one versus the other. Thus, with two-eyed seeing, this work will examine these two processes as fundamentally different but potentially more valuable when articulated together.

\subsubsection{Critical Race Theory and Tribal Critical Race Theory}

Critical race theory interrogates the relationships between power, race, and racism at the systemic and institutional level and critically examines the appearance of race and racism across dominant cultural modes of expression (Purdue University, n.d.). Critical race theory was developed in the 1970's by Derrick Bell, the first African American to teach at Harvard Law School (Delgado \& Stefancic, 1998). CRT offers the tools through which to examine "racialization, marginalization, and the role of critical race theorists in producing knowledge about societal inequities" (Ford \& Airhihenbuwa, 2010, p. 1390). As such, a primary objective of CRT is "to construct a reality through storytelling and counter storytelling" to oppose deficitbased stories and stereotypes (Delgado, 1989; Solarzano \& Yosso, 2002; Haynes-Writer, 2008).

As a specific branch of CRT, Tribal Critical Race Theory (TCRT) was developed by Lumbee scholar Bryan Brayboy in response to the increasing interest of Indigenous Peoples in 
CRT to unpack the complex relationships of race, racism, and power within communities, and utilizing CRT as "a mechanism to perform truth-telling — to speak back to colonization and oppression" (Haynes-Writer, 2008, p. 3). The development of Tribal Critical Race Theory provided a mechanism with which to examine the Indigenous-specific effects of colonization, especially as they pertain to institutional policies and laws, and to "generate truths about colonization in larger social and structural contexts, facilitating change" (Haynes-Writer, 2008, p. 3).

This theory is especially important in the context of this research as the deficit model is recognizable in many Canadian datasets that aim to statistically describe Indigenous people. Historically, Indigenous peoples in Canada have been subject to research under the guise of 'objective science' and most often producing results that claim to benefit the communities who partake, when the resulting findings instead validate or uphold stereotypical and deficit conceptions of Indigenous peoples (FNIGC, 2020). To counter this practice, critical race theory and tribal critical race theory (directly or indirectly) informs the research methods of Indigenous organizations such as the First Nations Information Governance Centre (FNIG), Inuit Tapiriit Kanatami (ITK), and the Rupertsland Centre for Métis Research (RCMR). These organizations seek to facilitate change for Indigenous Peoples in Canada by practicing the sharing of positive data and indicators of well-being to subvert deficit-based representations and utilize data and statistics to affirm positive stories. CRT and TCRT are therefore useful in their ability to facilitate the "unmasking, exposing, and confronting continued colonization within educational contexts and societal structures, thus transforming those contexts and structures for Indigenous Peoples" (Haynes-Writer, 2008, p. 1). 
The application of CRT and TCRT can be witnessed in various fields including law, public health, education, social science, and philosophy, to understand racialized risk factors and their social effects. With this thesis I aim to also apply CRT to the domain of science to explore how Western systems accommodate/disadvantage Indigenous knowledge considering historical colonialism and marginalization. I will also adopt the method of affirmative analysis over the deficit model, focusing on how open science can benefit Indigenous researchers and communities instead of examining how IK might not 'fit' the open science model.

\subsection{Defining Key Concepts}

In addition to these theories, there are several key concepts informing this research and, in this section, I will define these including local and traditional knowledge (LTK), Indigenous data, and Indigenous knowledge and data governance.

\subsubsection{Local and Traditional Knowledge (LTK)}

Scientists, Indigenous or non-Indigenous, may draw upon existing local and traditional forms of knowledge in their research. Local and traditional knowledge (LTK) is the culturally embedded knowledge systems, skills, and philosophies developed by societies with long histories of interaction with their natural surroundings, and are handed down from preceding generations (UNESCO, n.d.). LTK is "not a uniform concept across different Aboriginal cultures...it is a diverse knowledge that is spread throughout different peoples in many layers" (Henderson \& Battiste, 2000, p. 35) thus there is no static concept of what it means or what it is. LTK is different for every unique First Nations, Métis, and Inuit culture or community. The purpose and content of LTK varies, yet all forms are sacred and indicative of a culture's ways of knowing, history, values, and worldviews. Forms of LTK include, but are not limited to, 
folklore, language, holistic and medicinal knowledge, biodiversity, agriculture, and food: these forms of knowledge translate the histories, beliefs, ethics, practices, and traditions of unique cultures (Browne, 2012; Anderson, 2015). The term LTK is also commonly expressed as traditional knowledge (TK), traditional ecological knowledge (TEK), or Indigenous knowledge (IK), however, each of these terms denote holistic, experience based, often orally transmitted ways of knowing that differ from Western, academic epistemologies (Browne, 2012).

Throughout this thesis I will rely on the term Indigenous knowledge (IK) as the umbrella term for LTK, TEK, and IK.

The production and application of LTK is crucial to the self-determination of First Nations, Métis, and Inuit communities in Canada, whose ability to do so has tangible effects on communities. Sustainable development, cultural preservation, strengthened Indigenous identities, physical and mental wellbeing, and the ongoing success of Indigenous peoples are several demonstrate the importance of traditional knowledge (Anderson, 2015). LTK is relevant here as it may be expressed, applied, or studied in mainstream research, scientific or otherwise. In the context of this research project, LTK will refer to any form of traditional knowledge that is sacred and meaningful to an Aboriginal culture in Canada and central to the overall functioning of each individual community. This includes knowledge production that involve the creation of Indigenous data.

\subsubsection{Indigenous Data}

Scientific research may also draw upon various kinds of existing data to contextualize or support a research topic or may collect data and empirical evidence to substantiate claims about a research question. Depending on the research topic, this may include Indigenous data, or data about Indigenous peoples. There are various forms of Indigenous data, including statistical, 
administrative, and archival. Broadly speaking, Indigenous data could refer to "any facts, knowledge, or information about a nation and its citizens, lands, resources, cultures, and communities. Information ranging from demographic profiles to educational attainment rates, maps of sacred lands, songs, and social media activities" (Rainie et al., 2017, p. 1). However, it would be beyond the scope of this thesis to address all forms of Indigenous data in this way. For this reason, in the context of this thesis, I use 'Indigenous data' to refer to two categories of data:

1) any data collected by Indigenous peoples, communities, or organizations, to be primarily used by Indigenous peoples.

2) research data collected by outside researchers and Government about First Nations, Métis, and Inuit peoples in the context of a research project.

The former may refer to statistical, administrative, or archival data collected by First Nations, Métis, and Inuit communities towards the betterment of the community, where 'betterment' can include understanding phenomena within a community or its history, using data to determine where programs/assistance is needed, comprehensive or deeper understanding of populations, and planning purposes. For example, the First Nations Regional Health Survey (RHS), developed by the First Nation Information Governance Centre, is the first and only national health survey that conceptualizes a traditional understanding of health and well-being to assess the overall health of First Nations individuals in Canada. Similarly, Inuit Tapiirit Kanatami (ITK) collects statistical data using their annual Inuit Statistical Profile. Administrative data that are collected by Indigenous organizations to administer programs or services to those communities and can also be of a sensitive nature.

The latter refers to data collected by researchers in the context of a research project with or about Indigenous peoples. For instance, non-Indigenous researchers may enter an Indigenous community for the purposes of scientific research and/or data collection. In the context of open 
science, this research and data would likely be made open by default, and the data could be shared with any number of people for various purposes. This is overtly problematic, which is why here I will consider the implications of Indigenous data collection in OS research and ways in which there is the Indigenous governance of research and made openly accessible. Here I remain exclusive to research data and suggest that future research also examine the implications of open science on the governance of all forms of Indigenous data, including data collected by Statistics Canada, the National Household Survey, and any other forms of open data about First Nations, Métis, and Inuit peoples. However, for the purposes of this research project alone, the concept of Indigenous data is scoped to only examine the data described above, in part, because different forms of data have pre-existing governance structures and are subject to different legislation in Canada. The primary goal here, is to determine whether open science research (which draws upon Indigenous knowledge and includes or involves the collection of Indigenous data) poses a threat to the realization of data governance and data sovereignty for First Nations, Métis, and Inuit peoples.

\subsubsection{Indigenous Knowledge and Data Governance}

The United Nations Declaration on the Rights of Indigenous Peoples (UNDRIP), recognizes and affirms the Indigenous right to self-determination and sovereignty. This is also acknowledged in Section 35 of the Government of Canada's Constitution Act, 1982, the Report of the Royal Commission on Aboriginal Peoples (RCAP) and the Truth and Reconciliation Commission (TRC)'s Calls to Action. A component of self-determination and sovereignty is the "right and responsibility to exercise jurisdiction in relation to information governance - to protect and govern all aspects of their citizens and nation's information and data" (FNIGC, 2019). This means that Indigenous peoples in Canada have the right to exercise control and 
jurisdiction over their data and information, to manage them in a way that is consistent with their own laws, customs, and practices, and to prioritize the goals, objectives, and rights of Indigenous peoples (FNIGC, 2019). It reinforces the rights to engage in decision-making in accordance with Indigenous values and collective interests. This is the concept of Indigenous knowledge and data governance: the right to protect, govern, control, and exercise jurisdiction over their own knowledge and data in a way that is consistent with their own laws, customs, and practices, and prioritizes their own goals, objectives, and inherent rights as First Nations, Métis, and Inuit peoples.

This is particularly important for Indigenous populations considering the historical context. Indigenous peoples have been subject to governance systems, policies, and research performed and executed by non-Indigenous peoples (Phillips, 2017) and they have generally had very little control over the data collected about them (and the ways in which these data are used), and data have been used to administer programs and services that do not consider the goals, perspectives, or best interests of the people they are meant to address (Phillips, 2017; FNIGC, 2019). Additionally, data and information, including statistics and quantitative data (especially health data), have long been utilized to uphold deficit-based conceptions of Indigenous communities in Canada, presenting Indigenous peoples as inherently lacking. The collection of data and information by non-Indigenous peoples has caused considerable issues for First Nations, Métis, and Inuit peoples, and is an example of residual colonialism. Thus, the affirmation of the Indigenous right to self-governance (including data governance) and selfdetermination means that, after decades of being defined according to Western standards, negative data and deficit indicators, Indigenous peoples in Canada are reclaiming the right to define themselves and understand themselves on their own terms. 
The primary objective of this thesis is to determine how an open science framework could operate in a way that is supportive and respectful of this knowledge and data governance; not to argue that all Indigenous data and knowledge should be private and locked away. The primary concern, as I will demonstrate, is the ability of First Nations, Métis, and Inuit individuals and communities to be able to exercise authority and governance over their data and knowledge in an open science environment. For example: a research team contacts a community in the Arctic and wishes to conduct research in their native land, which could incorporate Indigenous ways of knowing, knowledge, and/or data, including traditions, population data, etc. The sharing of this information in open contexts - where consent is not required for public access/exchange may not always be in the best interests of those communities. Publication of this knowledge and data in the open domain could undermine the goals of data sovereignty, knowledge governance, and self-determination, however, there may not be an adequate mechanism to account for nonWestern knowledge. Considering the histories of data colonialism, therefore, research ought to be done to fully understand the potential benefits and disadvantages of sharing traditional knowledge and data in an open way and consider how knowledge and data governance can be exercised in the context of open science practices.

\subsection{Summary}

This chapter has provided the foundation for the research in this thesis by outlining the theoretical framework and key terminology. This hybrid theoretical framework relies on postcolonial theory, social construction of scientific knowledge, open science, two-eyed seeing, and critical race theory. By applying postcolonial theory, I aim to unpack the OS movement's underlying values and goals to reconsider the ways in which it embodies postcolonial values. 
This work is grounded in the notion that scientific knowledge is not always a neutral, objective representation of the world: instead, scientific research output is often influenced by the researcher's positionality among other issues such as funding, instrument choice etc. The goal of $\mathrm{OS}$, therefore, is to open the entire research process (from inception to publication) to ensure that results can be widely accessible, scrutinized, reused, and reliable. Applying the CRT and TribalCrit lenses to the OS theory will be important to critically scrutinize the potential for OS to transform the policies and institutions that embody colonialism and therefore present concerns for Indigenous Peoples in the research context. Finally, two-eyed seeing will inform my recommendations about co-developing a more Indigenous-focused OS framework in the Canadian context.

In the following chapters I will also rely on the definitions provided here to interrogate the potential for OS to align with the unique requirements to support Indigenous knowledge and data governance. Indigenous knowledge (IK) will refer to all forms of Indigenous-specific knowledge that may be used in research, while Indigenous data refers to any data collected by Indigenous Peoples for Indigenous Peoples, or data that are collected by researchers in the context of co-developed research projects. The terms Indigenous Knowledge and Data Governance (IKDG) refer to the inherent right to protect, govern, control, and exercise jurisdiction over their own knowledge and data in a way that is consistent with their own laws, customs, and practices, and prioritizes their own goals, objectives, and inherent rights as First Nations, Métis, and Inuit Peoples. 


\section{Chapter 3: Literature Review}

This research approach here is interdisciplinary, and I have selected literature from several social science fields including science and technology studies (STS), Indigenous and Canadian studies, international development, cartography, communication and media studies, public policy, and postcolonial studies.

I review academic and grey literature that includes policy documents, reports, and organizational literature. This chapter is structured as follows: 3.1 Decentralizing Western knowledge and decolonizing Indigenous knowledge explores how Western and Indigenous ways of knowing are experienced and articulated in public policy, education, and science domains, and considers how this might influence the process of decolonizing Indigenous knowledge. Section 3.2 Contextualizing and considering open science, is a brief and critical overview of the historical foundations of open science and the open science movement. Section 3.3 Postcolonial 
critique of standards explores how some scholars interrogate these, and 3.4 the state of codevelopment methods in Canadian research discusses the contemporary work which incorporates traditional knowledge in western research and identifies best practices and concerns of the co-management of Western and traditional data and knowledge. The synthesis of this literature will inform the content analysis that will follow.

\subsection{Decentralizing Western Knowledge, Decolonizing Science \& Indigenous Knowledge}

In this section I review academic journal articles that discuss the production of colonial knowledge and its embodiment in Western institutions, policies, and mainstream ways of thinking. I will here investigate how the processes of Western knowledge production can influence or impede the ability of Indigenous nations in Canada to divest from residual colonial power. This will situate Indigenous knowledge and data governance in the wider context of colonialism and data colonialism in Canada and locate open science within the historical context of science and colonialism.

It is beyond the scope of this thesis to explore a complete history of colonization in Canada; however, a brief exploration is important to situate this research. Prior to the arrival of European explorers and traders to Canada in the $16^{\text {th }}$ century, Indigenous peoples were living on the land and some on the Ice, with their own distinct cultures, ways of knowing, ways of doing, governance, and languages. After contact with colonizers, colonial power was asserted and with that came the imposition of their cultures, values, religions, laws, and policies on Indigenous peoples, and through physical conquest came the control of lands and trade routes (Wilson, 2018). Colonialism remains alive and well in Canada and is residual and manifests in various 
forms. Data colonialism, for example, refers to the mishandling and misuse of Indigenous data in ways that reinforce systemic oppression and unequal power relations, uphold deficit-based statistics, fail to benefit the Indigenous peoples whose data about them are collected, deprive Indigenous peoples of control over government-collected data, and neglect to consider the importance of data sovereignty (Open North, 2017). Data sovereignty refers to "Indigenous peoples' right to maintain, control, protect and develop their cultural heritage, traditional knowledge and traditional cultural expressions, as well as their right to maintain, control, protect and develop their intellectual property over these" (Taylor \& Kukutai, 2016) and is necessary for self-determination and governance.

Colonialism thus remains embedded in contemporary Canadian political, and economic institutions (Clogg, 2020). Whereas historical forms of colonialism "used nations, ethnicities, and races as its building blocks" and saw the conquest of people and land, (Viuru, 2006, p. 50) 'new colonialism' operates through institutions, policies, and ideologies within Western societies to prioritize Western knowledge as dominant, normative, and objective (Berkes, 1993; Magga, 2005; Viuru, 2006; Valsiner, 2006; Anderson, 2015; Snively \& Williams, 2016). The result is the imposition of Western knowledge systems and policies on Indigenous Peoples despite having their own knowledge systems and policies which are more culturally appropriate. This residual institutional colonialism is most easily recognized in fields such as education, healthcare, and science. There is now a focus on the process of decolonization, which includes the decolonization of knowledge and how it is produced. Decolonization is the process of deconstructing "the colonial ideologies of the superiority and privilege of Western thought and approaches" (Cull et al., 2018). Part of this process involves the inclusion and recognition of Indigenous forms of knowledge in Western form of knowledge production, although this work 
has frequently been considered as less legitimate than Western forms of knowledge even though Indigenous knowledge is gaining growing in importance (Verran \& Turnbull, 1999; Magga, 2009; Snively \& Williams, 2016). Decolonizing knowledge is consequently difficult in these environments where Western knowledge is actively reinforced as the dominant form of knowledge. This assumes first that Western knowledge - including scientific knowledge - is established, but may not be objective truth (Magga, 2009) and secondly the importance of interrogating how dominant knowledge is established, and by whom (Akena, 2012).

Dr. Radhika Viuru (2006) is a postcolonial scholar who interrogates the embodiment of imperial and colonial ideologies in education systems. In her foundational work, Postcolonial Technologies of Power, she argued that the underlying ideologies and values which shape institutional policies go largely unchecked, so the "discourses that surround the constitution for public policies need to be deconstructed for their participation in the new imperialism: the kind of colonialism that has less to do with the conquest of lands and properties and more to do with constructing human beings" (Viuru, 2006, p. 102). Viuru concluded that contemporary iterations of colonialism in education, frequently espouse "racist and colonial ideologies" (p. 101), and may be more insidious as they operate through global networks, infrastructures, and standards, which makes them more difficult to isolate and counter. Snively and Williams (2016) also found that this is still largely the case today, as the "colonialist educational system and the underrepresentation of Indigenous Science in the curriculum" (p. 12) corresponds to the urgent need for "Aboriginal (sic) peoples in science, technology, and health-related careers" (ibid). Indigenous knowledge and science are increasingly promoted: for instance, Indigenous knowledge and ways of knowing are progressively appearing in mainstream curriculum in Canada. Additionally, the Truth and Reconciliation Commission's Calls to Action, which is a 
report detailing 94 actions to be taken by the Government of Canada to advance reconciliation, has facilitated the government's commitment to include Indigenous knowledge in educational contexts. Despite these advancements, many challenges remain. Specifically, the scholars included here suggest that the underrepresentation of First Nations, Métis, and Inuit peoples in these domains is directly related to residual colonialism in public policy, and that these same institutions can consequently function as postcolonial institutions of Western knowledge production.

Decolonizing knowledge is challenging across domains, as is separating indigenous knowledge from residual colonialism. Indigenous health studies have shown promise, as this domain has consistently demonstrated that the promotion of Indigenous knowledge in public policy "can enhance overall Indigenous health outcomes through mutual recognition of differing worldviews" (Boot \& Lowell, 1), yet most conceptualizations of health literacy have remained demonstratively Western in terms of knowledge, paradigms, and practices (Boot \& Lowell, 1). Scholars Gordon R. Boot and Anne Lowell (2019), both scholars of Indigenous studies, conducted a qualitative content analysis of governance and public health policy documents across Canada, Australia, and New Zealand to gauge how Indigenous knowledge is incorporated and reflected in public health policy. The authors found that current health literature "frequently disregard the significance of Indigenous cultures, languages, and knowledges as strengths" which is unfortunate as these have directly benefited Indigenous peoples (Boot \& Lowell, 2). Their study demonstrated that the health policy documents they examined often included commitment to incorporating Indigenous perspectives and methods, but they generally did not include any specific information about how these perspectives and methods would be applied. The authors warned that there is a crucial difference between "passively recognizing and 
acknowledging the existence of Indigenous diversity, knowledges, paradigms, and practices" (Boot \& Lowell, 17) and promoting them. The authors concluded that, across domains, “acknowledging and promoting Indigenous knowledges and practices as legitimate...is imperative [as] it provides the foundation for governments, Indigenous communities, academics, and service-providers to work collaboratively" (Boot \& Lowell, 20) towards positive outcomes and greater recognition and legitimization of Indigenous knowledge.

The field of science is no exception. In their analysis of scientific and Indigenous knowledge, Helen Verran, who studies politics, policy, and governance of Indigeneity, and David Turnbull, whose work focuses on cartography and Indigeneity, conducted a postcolonial science study and argued that the Western-structured intellectual agenda "is nowhere more acute than in the assumption of 'science' as a foil against which all other knowledge should be contrasted" (Verran \& Turnbull, 1999, p. 137), acknowledging the dominance of Western knowledge within the domain of science. For instance, Indigenous peoples were practicing science long before modern science came to be (Magga, 2005), however, modern scientific research has predominantly favored Western forms of knowledge over traditional forms of knowledge (ibid) and Indigenous knowledge systems are understood to lack "the same authority and credibility as [Western] science" (p. 117). Some have further argued that the introduction of Indigenous knowledge into the mainstream domain can be seen as a challenge to the hegemony of Western science (Burgess, 1999) and consequently, Indigenous, or local science has historically been considered less scientific and therefore less credible in the Western world. Dr. Rohan Deb Roy, who studies the imperial history of science and medicine, found that the very field of modern science is a colonial enterprise. He argues that "colonial legacies continue to shape trends in science today" (Deb Roy, 2018, para. 20) and a startling majority of scientific 
knowledge is produced according to Western ideologies, values, and objectives. Others have argued that the process of decentralizing Western science and decolonizing Indigenous knowledge requires the recognition that what is considered 'science' in the Western frameworks is not more important or more credible than what is considered 'science' by Indigenous researchers or communities, despite their differing methods and exposure (Little Bear, 1993; Magga, 2005; Snively \& Williams, 2016). This means that the process of understanding how knowledge has been established - and by whom - is key. Deb Roy (2018) also found that locating the centralized production of scientific knowledge as Western - and by Western scholars - can be juxtaposed in such a way as to "accommodate non-Western perspectives and experiences" (Deb Roy, 2018, para. 34) and provides an opportunity to "rethink the dominant image that scientific knowledge is the work of white men" and to conceptualize it as something different (Deb Roy, 2018, para. 38). As Indigenous science and traditional knowledge become more common in mainstream research, science can be further decolonized in this way to be more inclusive and accommodating of non-Western ideals and values. For this reason, it is especially important to critically examine the underlying power relations between Western and Indigenous knowledge as they shape institutions, policies, and practices across domains including education, public health, and science.

Francis Adyanga Akena (2012), a scholar of African and Indigenous studies, explored the social positionality of knowledge producers to understand how Western knowledge is reinforced as 'legitimate' knowledge. Akena's critical analysis of the Production of Western Knowledge and Its Implications for Indigenous Knowledge and Decolonization sought to understand how the privileging of Western knowledge influences the pursuit of decolonization of Indigenous knowledge. Akena posits that knowledge is deeply influenced by "the individual and the society" 
(p. 607) in which it is produced, and further shaped by the "experiences and understandings" (ibid) of the people who adopt, transmit, and interact with it. This has unique implications for Indigenous peoples who are working to decolonize their own cultural knowledge and ways of knowing within the paradigm within which they live and produce knowledge. Akena asserts that Western knowledge becomes further entrenched and prioritized in institutional domains such as education, health, public policy, and science, which biases and "negatively influences the indigenous (sic) knowledge of a people; [how] this external imposition is disempowering and colonizing” (p. 606). Therefore, it is difficult to decolonize Indigenous knowledge.

The authors reviewed here have stressed the importance of exploring the social contexts of knowledge production to decentralize the production of Western knowledge as a first step towards including more diverse perspectives in contemporary institutions. It is therefore quite possible that open science, as an extension of regular science, could operate as a form of new colonialism to continue to shape the production and maintenance of dominant forms of knowledge. This might be the case as open sciences is facilitated by standards and principles which do not centralize Indigenous peoples as knowledge producers, as Western forms of science is not reflexive of its coloniality. It is my goal in this these to consider whether open science (as it is) might also be guilty of this fallacy, and, if not, to consider the ways in which open science can be shaped to avoid being a form of new colonialism.

\subsection{Contextualizing and Considering Open Science}

As discussed, here scientific knowledge is socially constructed and influenced. As such, if Western science is socially constructed and reflective of Western ideology, value, and objectives, it is possible that open science (OS) by extension might also reflect and underlying 
Western epistemology. Several scholars focus on the processes, advantages and disadvantages of open science, and there are scholars who critique the OS movement as potentially perpetuating Western hegemonic forms of knowledge production. In these sections that follow, I explore their work by describing the conceptual origins of OS as praxis before introducing it as a more inclusive iteration of the scientific method. I will close this section by examining common critiques of open science.

\subsubsection{Contextualizing Open Science}

Most scholars of open science agree that the practice and the concept emerged in the 1600s when the world's first scientific journal, The Royal Society's Philosophical Transactions, in 1665 was created (David, 2013; Friesike et al., 2014). Scientists could publish their research processes and findings enabling other scientists - and even non-scientists - access to research beyond the lab. This publication and related scientific publishing practices demonstrated the benefits of open access publishing as part of open science in two major ways. Firstly, open science provided access to research methodologies and scientific enabling others to reproduce and validate the results. In addition, replicability which is the ability for scientific research to be repeatable with the same results was then, and remains, a central tenet of the scientific enterprise (Hanwell, 2018). The second major benefit of open access publishing meant that research results could be disseminated to a wider audience, sharing the potential benefit of knowledge with as many people as possible. Thus, open access publishing and the practices of open science was, and remains, driven by the need for transparent and credible science to be used for public good.

What is also commonly discussed in the literature, is that from the $18^{\text {th }}$ century onward the scientific enterprise increasingly becomes commodified (Smith, 2006; Valsiner, 2006; Radder, 2010; David, 2013; LaFuente \& Estalella, 2016). This is, in part, due to the increased 
popularity of patents in industry and agriculture after the depression during the end of the $18^{\text {th }}$ century. As the industrial revolution transformed economies by introducing large-scale industry and mechanized manufacturing, so too did it push scientific knowledge into the marketplace (Radder, 2019). This continued into the $19^{\text {th }}$ and $20^{\text {th }}$ centuries. Marcos Barbosa de Oliveira's work on the commodification of science suggests that scientific knowledge gained significant economic importance in North America after World War II and that the changes in the "distribution of scientific knowledge go along with the changes in the economy and society as a whole" (de Olivera, 2012, p. 2465) during this time. It is the birth of what de Olivera terms 'innovationism' that sits at the "core of the main neoliberal strategy to promote the [commodification] of science" (de Olivera, 2012, p. 2469). Hans Radder, a scholar of science and philosophy, also notes the confluence of this phenomena with the transformation of academic knowledge and science in universities, specifically, resulting in modern neoliberal commercialization of science and technology (Radder, 2019).

This naturally continued to affect open access publishing. It is widely agreed that open access (OA) publishing was a reaction to the corporate publishing industry that made scholarly research, particularly scholarly journal articles, exclusive and difficult to access. While the number of journals and journal articles were increasing in the mid 1990's (Suber, 2009) during the era of Web 1.0 - or the initial evolution of the World Wide Web - the price to access journals and their content was rising, which resulted in decreased accessibility. Notably, cultural psychologist and scholar Jaan Valsiner discussed in their work on the social contexts of open access that "openness [was] changing into its opposite - closing through patenting and economic access barriers - precisely at the time when new information technology allows for speedy and unlimited access" (Valsiner, 2006, p. 1). OA was the resulting movement to ensure that the 
results of scientific research were more readily available to the public, allowing unrestricted access to scholarly research and journal articles. OA publishing activity increased significantly in the 2000's during the first stage of the World Wide Web's development (Suber, 2009).

Despite increased output made available by open access publishing, the field of science has remained mostly closed. Throughout the second evolution of the World Wide Web - or Web 2.0 - which enabled and facilitated more citizen science, crowdsourcing, and similar modes of 'free' scientific knowledge production, commodification of scientific knowledge and closed science has persisted. Regarding academic and federally funded scientific research, Marcus Hanwell, an open software engineer whose work focuses on open science and open science software, (2018) argued in recent years that the practice of open and transparent science has become less common, as "more and more science [relies] on closed elements, ultimately leading to errors that are more difficult to spot without full sharing of data, methods, and publications" (Hanwell, 2018, para. 2). A primary example of this is the exclusivity of most scientific journals which require users to either pay or subscribe to them, though it is also true that more and more journals have recently begun to offer their content for free (LaFuente \& Estalella, 2016). The consequence of a lack of openly available and accessible methods, data, and publications is that it becomes more difficult to scrutinize, share results, validate results, and extend the bounds of existing scientific knowledge (David, 2013; Shapin, 2015). The resulting concern over the integrity of the scientific discipline, coupled with a staggering reproducibility crisis, has also led to a kind of second wave of open science enthusiasm.

\subsubsection{Impetus for Open Science}

Most OS scholars agree to some extent that there is a reproducibility crisis in science and other domains. Recognized across disciplines, the reproducibility crisis began with a claim made 
in 2005 by Dr. John Ioannidis, who argued that "most published research findings in his own field of medicine were false" (Randall \& Wesler, 2018, p. 11). In a report by the National Association of Scholars (NAS), authors David Randall and Christopher Welser (2018) describe how "improper research techniques, lack of accountability, disciplinary and political groupthink, and a scientific culture biased toward producing positive results together" (p. 11) have resulted in a reproducibility crisis, whereby scientific results often cannot be reproduced or verified in further studies. What started as a claim to the field of medicine came to expose a crossdisciplinary issue which compromises many other scientific fields (ibid).

The authors state various reasons and improper scientific practices that could explain this issue. For instance, pre-conceived goals early in the scientific process could lead researchers to "look for correlations until they find a spurious 'statistically significant' relationship" (p. 11). Consequently, researchers may "consciously or unconsciously bias their data to produce desired outcomes" (p. 11). The current scientific environment is also conducive to irreproducible science. For example, uncontrolled research freedom and few restraints on research projects allow "more opportunities for them to go astray" as "oftentimes researchers can alter their methods midway through to pursue statistically significant results" (p. 12). Absence of openness, whereby researchers neglect to share methodologies and data, and a premium on positive results, meaning "modern science's professional culture prizes positive results far above negative results, and also far above attempts to reproduce earlier research" (p. 12), contribute to this problem. The report demonstrates an alarming pervasiveness of these inconsistencies:

A 2015 article in Science that presented the results of 100 replication studies of articles published in prominent psychological journals found that only $36 \%$ of the replication studies produced statistically significant results, compared with $97 \%$ of the original studies (Randall \& Wesler, 2018, p. 12). 
The replication crisis, which primarily affects the fields of medicine and the social sciences, has been acknowledged by many (Schooler, 2014; Shapin, 2016; Loken \& Gelman, 2017; Shrout \& Rodgers, 2018; Renkewitz \& Heen, 2019). In response, NAS recommended that: researchers make their data open for public inspection once they have published their results; data should be open by default and automatically time-stamped; and researchers should adopt standardized descriptions of research materials and procedures (Randall \& Wesler, 2018, p. 13-14). Thus, the process of open science, which relies on the increased transparency of the scientific method, sharing results via open-by-default specifications, and following open science standards, is often considered a solution to the problematic issues of modern science just discussed because it establishes a set of best practices to facilitate openness throughout the entirety of the research cycle (Schooler, 2014; Loken \& Gelman, 2017; Randall \& Wesler, 2018). Also, by removing traditional barriers to access, open science can transform knowledge ecologies and facilitate a more diverse science which is inclusive of voices who have been traditionally marginalized in the Western science domain (Smith \& Seward, 2020, Grahe et al., 2020).

\subsubsection{Critiquing Open Science}

Despite the ideals of a more transparent and inclusive scientific movement, many scholars have considered how open science might perpetuate issues of inaccessibility, exclusivity, and colonialism in the science domain. The ideals of open science are about sharing knowledge across different domains as early as it is practical in the research process (Nielsen, 2011). Leslie Chan who studies the geopolitics of knowledge production, open science, and knowledge equity, has examined centrality of Western knowledge in the context of open science mostly in a developing country context and argues that, while the idea of OS promises to share diverse knowledge and, in many cases, facilitate citizen science to include more diverse 
perspectives, important questions remain such as "whose science is being opened? Who is going to benefit from these new framings and practices? What are the risks? Will this lead to equality and equity of knowledge access and production by researchers in unequal settings?' (Chan, 2019). These are important questions and are related to the decentralization of Western knowledge, decolonizing science, and valuation of Indigenous knowledge. Despite the hope of open science as a solution to Western-dominant knowledge producing practices in the sciences in general, and providing opportunities for diverse voices, several scholars caution that OS should not be idealized outside of the history of scientific colonialism (Valsiner, 2006; Piron, 2018; Chan, 2019; Smith \& Seward, 2020). It becomes clear that while OS has benefits, it is situated in the practices of Western science and stems from and in some cases remains steeped in colonial practices. This may be attributed to the fact that traditional barriers to accessing and contributing to scientific endeavors are not eliminated simply because science adheres to OS. Conceptually, OS and OA imply that research can be more accessible to the masses as a public good, with the elimination of access fees. The corollary is that more people can participate in the production of knowledge because they can access current research, build on existing knowledge, and share their own research and perspectives. It is also true, that financial barriers are not the only barriers stopping researchers from accessing research output or participating in the scientific process (Valsiner 2006; Carillo \& Papagni, 2014; Gagliardi, Cox \& Li, 2015). Jaan Valsiner (2006) an early critic of open systems after examining the social context of knowledge producing institutions, concluded that they continued to promulgate Western knowledge producing processes.

Papia Sengupta (2020) in her work on postcolonial open access, argues that OS entrenches academic colonialism. Academic colonialism, which is the notion that "authors and 
academics from the developed powerful countries [have] better access to funding and institutional support due to their location and have better chances to get their works published" (Sengupta, 2020, p. 204) is agreed by many to be the biggest disadvantage of OS since Western scientists have better access to funding and institutional support and thus have more opportunities to publish and to pay OA publishing fees. Since scientific knowledge is produced in the West, OS may serve to make "the work produced at the center of the world more accessible" (Sengupta, 2020, p. 109). Gagliardi, Cox \& Li (2015), who examined operational and systemic barriers to open science adoption in their study of institutional open science also found that the uptake of, and participation in, OS can be limited by systemic issues including "difficulties in assessing the quality and rigor of research contributions, lack of skills and/or time to contribute to the open science movement" (p. 107) which results in the slower uptake of OS on a global scale. These barriers are unevenly distributed along sociodemographic lines, meaning that researchers, communities, and institutions who are financially limited cannot participate in OS in a meaningful way. OS might therefore reinforce the dominant Western ideologies and forms of knowledge since access to infrastructure and capital remain a central component to participation and inclusion science in general, including OS (Valsiner, 2006; Gagliardi, Cox \& Li, 2015; Chan, 2019) leaving only those who can afford to participate will be heard (Valsiner, 2006; Gagliardi, Cox \& Li, 2015; Sengupta, 2020). Financial barriers continue to hinder "the cultural changes necessary for embracing the principles and the practices of open science" (Gagliardi, Cox \& Li, 2015, p. 2).

The literature reviewed thus far, provided a contextual history of OS, emphasizing its fundamental epistemology, which is that science should be transparent and credible, and research outputs are a public good that should be shared with the public. OS is also largely considered to 
be a more democratic and diverse form of doing science and is often positioned as a solution to replicability issues of modern science. Several others have also argued that OS might also reinforce dominant Western scientific knowledge production. The consequence is an OS terrain that lacks diversity, inclusive perspectives, and that ultimately fails to transcend the dominant colonial knowledge production paradigm. In sum, OS practitioners ought to critically reflect on their practices, and consider who makes the rules, and whose knowledge stands to be prioritized, especially in the realm of scientific publishing (Valsiner, 2006; Carillo \& Papagni, 2014;

Gagliardi, Cox \& Li, 2015; Piron, 2018; Chan, 2019; Sengupta, 2020).

\subsection{Postcolonial Critique of Standards}

Standards are an important aspect of OS as they play a central role in the production and dissemination of scientific knowledge. A standard is an established norm considered by an authority or by general consent as an approved model, rule, or principle (Merriam Webster, n.d.). In other words, a standard is a tool with which things can be normalized or made similar according to a set of requirements or rules. As described by the International Organization for Standardization (ISO), standards can be thought of as "a formula that describes the best way of doing something" (ISO, n.d.) and also a set of tools that "work in the background of our daily lives to make things easier, safer, and better" (ISO, n.d.).

In the context of data specifically, standards are used to measure, record, and transmit data, and "allow data collected at one place and time to be compared meaningfully with data collected elsewhere and/or at other times" (Edwards, 2004). OS relies on de-facto standards for open data sharing, which are principles or protocols that are accepted or practiced but may not be officially recognized or approved by an official standards organization (Carpenter, 2012). FAIR 
principles, for instance, provide guidelines for making open digital assets findable, accessible, interoperable, and reusable (Wilkinson et al., 2016), but scientific endeavors need not abide by FAIR principles to be considered OS, as there is no governing body to enforce their implementation. Organizations such as UNESCO, are trying to change this with their Recommendation on Open Science to set an international standard instrument on OS before the adoption by Member States in 2021 (CODATA, n.d.). Up and until this time, OS has been practiced by institutions, governments, and organizations by relying on best practices rather than standards. As such, when I refer to 'open science standards' in this thesis I am referring to defacto standards, guiding principles and rules to facilitate the practice and adoption of OS and not those developed by a standards body, instead I will refer to standards developed by the Research Data Alliance, CODATA, and other organizations in Canada.

In terms of Indigenous knowledge and data governance, standards are about different sets of principles developed by First Nations, Métis, and Inuit organizations to encourage best practices for knowledge collection, production, and dissemination. For example, the CARE principles, developed by the Global Indigenous Data Alliance, aim to complement the FAIR principles by incorporating Indigenous-specific goals of data management, collection and governance including collective benefit, authority to control, responsibility, and ethics (GIDA, 2019). Individual First Nations, Métis, and Inuit communities may have their own standards for data and knowledge collection and sharing, especially in the context of research.

In his 2004 text, A Vast Machine, Paul Edwards (2004) traces the evolution of meteorological standards and their role in "measuring, recording, and transmitting data...allowing data collected at one place and time to be compared meaningfully with data collected elsewhere and/or at other times" (p. 827). Edwards contends that standards are social 
technologies which are instrumental in the process of communication, effectively interoperating systems, and even detecting major trends within systems (Edwards, 2004). Many scholars have accepted this rationality of standards as social technologies, and some have consequently critiqued standards with a postcolonial lens. This is because the process of standardization, not unlike Western science, means normalizing according to a particular set of values, ideals, and priorities; and in Canada this most often means according to Western ideals. Standards act to homogenize, and to make things the same: if standards are also social technologies, they are almost certainly reflective, to some degree, of the environments in which they are developed. Some scholars (Viuru, 2006; Russell, 2014; Sengupta, 2020) therefore argue that standards can reinforce Western ways of doing and knowing, and accept that standards are inherently political (Viuru, 2006; Russell, 2014; White, 2019).

Viuru (2006) in her work on postcolonial education, suggests that Western standards function as postcolonial technologies of power and cautions against the practice of standardizing according to Western ideals: though Western standards are touted as objective, they represent the "ultimate imposition of rampant scientifico, [and] corporate capitalism" (Viuru, 2006, p. 49). Similarly, Sengupta (2020) states that Western scientific standards reflect the ideals and knowledge of the Western world and "set[s] the rules and standards of the so-called game of what is publishable" (p. 204), therefore indirectly influencing "what is 'scientific' and [what] is significant for publication" (ibid). Standards, then, might be new forms of colonialism as these structure content and those who participate in OS, especially if the standards prevent diverse groups from participating in their creation and by lacking culturally relevant concepts and flexibility. There is presently however, to the best of my ability to search, no literature on the postcolonial critique of open science standards. A gap I hope to address in this thesis. 
Scholars Dr. Andrew L. Russell and James Merricks White have in their work politicized standards. Russell's (2014) work focuses on the history of technology and computing, describing the historical and theoretical roots of open standards in the context of network systems, and addressing the politics and ideological values of standardization in new digital networks. Russell (2014) suggests that the development of open standards was originally meant to counter the centralized control of communication information networks, such as Bell and ARPANET, which had been ongoing for most of the 20th century. The move to design new digital networks in the 1970's and 1980's embraced the concept of openness to frame open systems considering idealized views of innovation and participatory democracy. The ideology of openness he indicated was a "utopian transformation in which power hierarchies are flattened, secret activities are made transparent, individuals are empowered, and knowledge itself is democratized" (p. 2). This idealized version of openness in relation to standards remains today, suggesting that open systems will result in "transparency, public participation, and collaboration" (ibid). However, says Russell, the emphasis on openness discourse continues to shroud the fact that these open systems are still heavily dependent on standardized hierarchies of control. And this is the case since "standards, if they are noticed at all, simply appear as authoritative, objective, uncontroversial, and natural" (p. 16), though they have "distinct political, economic, and cultural dimensions" and "embody the dominant values and assumptions" (ibid) of the social environments in which they are developed. Standards, like technologies, and science are "both socially constructed and society shaping" (p. 3) and therefore facilitate silent hierarchies within open systems which are assumed to be void of them.

In his own interrogation of standards, White (2019), identified standards as a site of important political engagement, and argued that "standards-making is an intensely political act, 
during which ideas about how the world is, and how the world ought to be, inevitably come to affect the final artefact" (White, 2019, p. 41). He examined smart city standards as a political tool in 3 major ways: he stated that "standards function by propagating ideas, materials and techniques; by steering outcomes towards desirable goals; and by assuring city leaders and decision-makers" (p. 6). Like the authors mentioned thus far, White agreed that standards "embody ethics and values" (p. 21) and are "uneven in their social distribution", meaning that standards are developed by certain people for certain end results, and may have different impacts on different actors. Citing a paper by Susan Leigh Star (1990), White explains that "standards create difficulties for people who do not fit within their assumed categories, and these categories can be difficult to challenge and overturn" (White, 2019, p. 20) and this concept is important here. These critical assessments identify the underlying power structures of standards and the knowledge frameworks with which they are developed, yielding important implications regarding who OS is for and is about.

This political understanding of standards from Viuru, Sengupta, Russell and White is very useful here because when it comes to data, information, and knowledge for and about Indigenous peoples in Canada, standards may also be infrastructures of colonial power, and be inherently yet silent social and technical political tools (Viuru, 2006; Russell, 2014; White, 2019; Sengupta, 2020). While some Indigenous standards are emerging, it is important to consider whether OS standards are flexible enough to incorporate, or make room for, Indigenous models. In the following chapter I will critically assess OS standards and compared these to local and traditional Indigenous principles of knowledge governance practices. For example, the ideas of OS, especially open standards, are normally viewed from a Western lens in that, despite being more open and accessible, the goal of OS is to disseminate scientific outputs to as many people 
as possible and to completely open the scientific process from conception to publication. This is contrary to ideals of knowledge governance in Indigenous communities; as not all knowledge should be widely broadcast, for reasons including individual privacy, community harm, biodiversity risk, and others. Here I accept that standards are arbiters of power within an OS and scientific system, and I question their function and power to reinforce dominant ideologies and critique their overall goals and consequences in terms of Indigenous knowledge and data governance.

\subsection{The State of Co-Development Research in Canada}

The co-development of research methods refers to the application of Indigenous ways of knowing and traditional knowledge alongside Western methods. Co-developed research in this case can include research by non-Indigenous researchers in collaboration with Indigenous peoples, or research done primarily by Indigenous researchers who draw upon Western methods with their own worldviews. Western scientific research is increasingly informed by more Indigenous ways of knowing, worldviews, and knowledge. This integration of Western and Indigenous science in co-developed research requires an understanding of the distinctions between the two knowledge systems to appreciate the strengths and benefits of applying them together. The differences are heavily documented (Wolfe et al. 1991; Cajete, 2000; Barnhardt \& Kawagley, 2005; Little Bear 2009; Berkes 2014, 2017); however, the modern movement towards open science necessitates the renewed exploration of this discourse.

Ray Barnhardt and Agnayuqaq Oscar Kawagley, both scholars of anthropology with a focus on Indigenous studies, analyzed the similarities and differences of "traditional Native (sic) knowledge systems and Western science" (Barnhardt \& Kawagley, 2005, p. 16) in their work, 
Indigenous Knowledge Systems and Alaska Native Ways of Knowing. Their comparison succinctly captures the nuances of each form of science while acknowledging their shared organizing principles (universe is unified, body of knowledge stable but subject to modification), habits of mind (honesty, inquisitiveness, perseverance, open-mindedness) skills \& procedures (empirical observation in natural settings, pattern recognition, verification through repetition, inference and prediction), and knowledge (plant and animal behaviour, cycles, habitat needs, interdependence, position and motion of objects) (Barnhardt \& Kawagley, 2005). Their comparison suggests that Indigenous knowledge systems in science are more holistic, emphasize the practical application of skills and knowledge, and often rely on the communication of metaphors and stories to convey values and proper behaviour. Conversely, Western science is grounded in skepticism, emphasizes understanding the 'how' in research, utilizes hypothesis falsification and relies on quantitative written word (ibid). Despite their differences, Barnhardt and Kawagley insist that recognition of their similarities, and the subsequent application of collaborative research, is critical for Indigenous Peoples to "find value in what emerges and to put new insights into practice as a meaningful exercise in self-determination" (p. 21). This early comparative work has remained relevant as Indigenous and Western science continue to be bridged in scientific research.

In the last decade an increasing number of research projects have incorporated codevelopment of methodology approaches in this way (Turnbull, 2009; Armitage et al., 2011; Pulsifer, 2014; Boot \& Lowell, 2019; Alexander et al., 2019). A 2019 qualitative content analysis by Alexander et al. (2019) in their study of Bridging Indigenous and science-based knowledge in coastal-marine research, found that, between 2000-2005, there were very few published articles that "integrate and/or include Indigenous and science-based knowledge in 
Canada" (p. 1) and fewer still published prior to 2000. The occurrence of research incorporating Indigenous and science-based knowledge systems increased in 2006, and the "annual volume of articles published remains relatively consistent from 2006-2018" (Alexander et al., 2019, p. 7). Most published articles during this time have first authors affiliated with academic institutions and government organizations, respectively, while approximately $21 \%$ of the publications were authored by individuals who represent "Indigenous communities, organizations, and/or governments" (p. 7). Co-development methods and best practices are therefore still emerging, largely from the academic domain, by scholars who may or may not be Indigenous.

\subsection{Benefits}

As traditional knowledge (TK) becomes more widely accepted as credible knowledge in mainstream science, scholars who work in this domain have argued that co-development methods can be mutually beneficial for all involved parties (Battiste, 2002; Magga, 2009; Taylor, 2013). Perhaps the most widely documented benefit of co-development is the preservation of TK including cultural artifacts, language, stories, oral history, and other aspects of Indigenous culture (Magga, 2009; Browne, 2012; Ngcobo \& Obono, 2013; Taylor, 2013). TK is increasingly appearing in digital environments using technological infrastructure for the purpose of cultural preservation and this is important since TK is "disappearing everywhere as a consequence of the many centuries long cultural war against Indigenous cultures and lack of recognition” (Magga, 2009, p. 7). This is echoed by many for example: Ngcobo \& Obono (2013) argue that TK "is at the risk of extinction due to the difficulties to preserve it as most of the IK largely remains undocumented" and the United Nations (UN) have declared that there is an "urgent need to enhance the intergenerational transmission of Indigenous knowledge" (UNESCO, 2008, para. 3). Consequently, a major benefit of this work is that "knowledge of land, sea, ice, weather, and 
additional natural phenomena can be shared with community members, researchers, and appropriate parties" (Browne, 2012, p. 4). The benefits of sharing TK in online environments also aligns with the goal of OS's sharing of knowledge for the public good.

Most significantly, the digital preservation of TK research has been a focus of the Geomatics and Cartographic Research Centre (GCRC) in the Department of Geography and Environmental Studies at Carleton University. The GCRC is a research center with more than 20 formal agreements and research partnerships with various communities and organizations in the Arctic including Indigenous organizations like Inuit Tapiirit Kanatami (ITK), Gwich' in Social and Cultural Institute, and communities in the Arctic region (GCRC, n.d.). Much of the work from the GCRC involves digital mapping for the archiving and preservation of local and traditional knowledge through the application of methods such as cybercartography and geospatial information management.

Looking to the field of cybercartography provides a wealth of information about how to do successful co-development and offers best practices for handling, managing, and sharing traditional Indigenous knowledge and data that have been digitally translated. Cybercartography is a mutually beneficial method as it disrupts the traditional privileging of Western knowledge by "allow[ing] the presentation of different ontologies or narratives on the same topic without privileging one over another" (Taylor, 2019, p. 57). The process of knowledge production is more user-based, meaning that cybercartography can be collaborative and more accessible than traditional map-making since one need not be a learned cartographer to plot data with maps and atlases. Cybercartography represents an example of collaborative, participatory methods and is a form of OS praxis. 
Indigenous knowledge, digital data and Western research are not without challenges. In their 2013 publication Legal Issues in Mapping Traditional Knowledge, for instance, Engler, Scassa \& Taylor (2013) identified several key benefits and challenges to preserving local and traditional Indigenous knowledge in online formats. Projects such as "creating atlases of traditional place names or recording the patterns of movement of sea ice" (p.1) are important to Indigenous communities who view the project as an opportunity to record their sacred LTK in a way that transcends linguistic and cultural barriers. The authors also note that this approach is beneficial because it gives Inuit peoples the opportunity to collaborate as partners in research that will directly benefit their communities. The Nunaliit Atlas Framework is another example that was developed by the GCRC and is an open-source software platform that facilitate communitybased collaboration in online atlas creation (GCRC, n.d.). The mapping technology interface allows communities and individuals to "input their data and develop interactive mapping and atlases to tell stories and highlight relationships between many different forms of information" (GCRC, n.d., para. 1). The atlas demonstrates the potential for OS to accommodate the interests and goals of Indigenous knowledge and data governance, as it allows "those who enter the data [to] autonomously decide how they want those data represented and who can use and access them" (Taylor, 2019, p. 63).

The acceptance of TK alongside Western science methods can also lead to increased "principles and guidelines for protecting Indigenous knowledge and its pedagogies [and] shared capacities that can alleviate poverty and create sustainable development" (Battiste, 2002, p. 6). Applying TK in scientific research also helps to raise the social value of TK as a legitimate system of knowledge, and consequently "Indigenous scholars [can] generate the necessary intellectual space to create a conceptual and analytical framework for its development" (ibid). 
These are but a few examples, that document many benefits derived from co-development approaches in scientific research, suggesting that there are several benefits that can be derived from OS practices that have a post-colonial and LTK focus.

\subsection{Challenges}

Despite these benefits, there are many challenges, and many hesitate when it comes to TK, Western methodologies, and co-development. The First Nations Ethics Guide on Research and Aboriginal Traditional Knowledge, produced by the Assembly of First Nations, expresses this general feeling of uncertainty among Aboriginal (sic) people when it comes to the growing application of TK in research. Though this shift represents an opportunity for collaboration and renewed approaches to policy, research and data management, there remains challenges:

Aboriginal people are concerned about the appropriate use and protection of their knowledge. Many deem integrationist research and implementation methods as another form of colonization and exploitation, where knowledge is categorized into hierarchies and [Aboriginal knowledge] can be devalued, exposed, abused or used against Aboriginal empowerment to self-govern their resources (Assembly of First Nations, n.d., p. 3).

This uncertainty can be compounded by the unfamiliarity of Indigenous Elders with information communication technologies. Browne and Ljubicic (2019) noted that the "consequences of placing information on the Internet may not be clear to Elders who are unaccustomed to computers" (p. 118). Furthermore, scholars doing cybercartographic work with the GCRC have identified several red flags which fall into two broad categories of concern: issues regarding informed consent and limited control of reuse of traditional knowledge and data (ethical concerns), as well as intellectual property and knowledge exploitation (legal concerns). 


\subsection{Ethical Concerns}

There are several ethical considerations regarding the use of traditional knowledge including ecological, biodiversity, medicinal, and even historical knowledge - and Indigenous data in research. Timothy DiLeo Browne's (2012) a Canadian studies scholar who interviewed Inuit elders and research contributors as part of his work with the GCRC, in his work on Consent and Ethics on Local and Traditional Knowledge in the Internet Era: Some Key Questions examined these issues by focusing on the key ethical concerns regarding the digitization of local and traditional knowledge (LTK) in the creation of web-based atlases by the GCRC. Browne emphasized the attention paid to obtaining the ethical requirements necessary as per institutional and community guidelines, describing how GCRC researchers "sought proper ethics clearance from the university, obtained Arctic research permits, consulted with communities, and used a thorough consent form, available in both English and Inuktitut" (p. 4) and the final product was returned to the communities who took part in the atlas creation. Despite checking off all these boxes, Browne acknowledges that these processes alone do not "adequately address the potential ramifications of placing personal and community information on the Internet" (p.4) as sharing information in the online domain allows access to this knowledge by a broad range of people with any number of interests. Browne also flags the issue of third-party possession as potentially problematic, since recording information in online formats requires technological infrastructure and technical expertise that may be beyond what is available to the community. The resulting reliance of communities on "outside agencies - such as the GCRC" (p. 37) necessitates a level of consent and relationship not accounted for in the standard ethics and consent forms. The GCRC, therefore have agreements in place to manage data in the south, Ottawa, on behalf of communities in the North, and are dedicated to capacity building so that in the future, with better 
ICTs and skills, people in the North can manage these themselves, but that is by no means the norm. In sum, there are many concerns which arise when preserving or sharing LTK in online formats that may still arise even when the researchers have taken every necessary step towards ethical research, further demonstrating that Western frameworks are not necessarily designed with Indigenous interests in mind.

This is especially the case when this research is openly published on the Internet. Scholars (Browne, 2012; Engler, Scassa \& Taylor, 2013; Browne \& Ljubicic, 2019) note that sharing TK digitally raises several questions about consent, ethics, and sovereignty, as the control of information is more difficult to exercise, and

Given corporations' growing interest in the natural resources of the Arctic and the concomitant rise in government concern about claims to Arctic sovereignty, such maps may also be of interest to a broad range of actors and for a variety of purposes. Because these projects rely heavily upon, and record, oral knowledge, and because they convert such knowledge into highly malleable and easily disseminated digital content, they raise challenging issues around informed consent, intellectual and cultural property, and privacy (Engler, Scassa, \& Taylor, 2013, p. 189)

Engler, Scassa \& Taylor (2013) argue that it is important to develop protocols for adequate consent regarding data collection use, and the application and management of Indigenous knowledge. Open sharing presents unique problems with the exercise of informed consent because partners may consent to the initial research project, and even the sharing of the content to a database or network, but the unhindered distribution of research in open online databases limits the ability of Indigenous peoples to consent every time their knowledge is shared (Engler, Scassa, \& Taylor, 2013). These concerns are echoed by Timothy DiLeo Browne and Gita Ljubicic (2019), a geographer who had conducted research and built atlases with Inuit in the North, and whom has bridge the Western science and Inuit TK in sea ice research, both 
explored the ethical considerations of using cybercartography and digital atlases to preserve and share traditional knowledge, especially when this information "incorporate[s] personal, social, and cultural knowledge on a wide range of topics and require[s] careful consideration of consent, data storage, data access, and appropriate dissemination" (p. 117).

Key recommendations made by these authors include recognizing the importance of reciprocity in the research process, ongoing trust between researchers and participants, and acknowledging that a "uniform approach may not work" (Browne \& Ljubicic, 2019, p. 120) and protocols should be adaptable due to the varying needs of Indigenous communities and the varying forms of TK across cultures and communities. It is thus crucial that any kind of standards-based-solution for collecting informed consent be "driven by Indigenous peoples, communities, families, and organizations" and "must be reflexive and consider cultural, historical, and geographical contexts rather than focusing on technical aspects of standards" (ibid). Browne (2012) recommends that one of the most important steps that can be included in the initial consent process is to make it clear that "consent to place data on the Internet cannot really be withdrawn, once the data are online" (p. 37). Browne concludes his work "on a note of uncertainty" (p. 37), explaining that several questions remain unanswered regarding best practices for obtaining adequate informed consent with research that will be distributed online and in open domains. This uncertainty has been echoed by others (Scassa, Engler \& Taylor, 2013; Browne \& Ljubicic, 2019). However, there is work that outlines best practices for handling and managing Indigenous knowledge and data. In their reflective piece on lessons learned from developing an atlas of sea ice knowledge, Peter Pulsifer, D. R. Fraser Taylor and Amos Hayes, researchers and lab manager at the Geomatics and Cartographic Research Centre at Carleton University, and Gita J. Laidler, from the Department of Geography and Environmental 
Studies at Carleton University, sought to contribute to the development of an Indigenist data management program. Their primary findings suggest that the development such a program is necessary if major research initiatives, such as the International Polar Year, are to respectfully and meaningfully engage with Indigenous knowledge systems alongside Western scientific methods (Pulsifer et al., 2011). Furthermore, they suggested that such a system need be rooted in "respect, reciprocity, and responsibility" (p. 121) to facilitate ongoing trust among Indigenous partners and maintain lasting, respectful relationships that are reflective of true collaboration. Perhaps most significantly, Pulsifer et al. concluded that co-development of an Indigenist data management program is the key to its success, as it is only through "dialogue with community members, researchers, legal experts, technical experts and the general public that an Indigenist DM program can be envisioned, articulated and realized" (p. 121) in a way that is impactful for Indigenous collaborators.

The issues raised by these scholars and others are broad and cannot be solved with a single mechanism for more appropriate informed consent, nor can a single framework be applied for all Indigenous communities involved in research: though some interesting work has attempted to provide insight in this regard. Most significantly, a proposal for an Open Licensing Scheme for Traditional Knowledge, developed by the GCRC in collaboration with the Canadian Internet Policy and Public Interest Clinic (CIPPIC), sought to offer a framework through which values for TK were mapped with other existing licenses to bridge the gap between existing IP laws and TK. For instance, the work proposed license terms such as "Give Back/Reciprocity (GB)" which would "oblige all users of the knowledge to 'give back' the research they create to the communities, including: providing relevant communities with copies of any research articles or reports made; providing access to any projects created using the knowledge; or giving the 
communities a portion of remuneration received from publication" (GCRC \& CIPPIC, 2016, p. 13) and "No Sharing" (NS) or "Personal Use Only" which would "prevent users from sharing the knowledge they acquire with anyone other than a specified set of communities, groups of communities, and/or groups of researchers" (GCRC \& CIPPIC, 2016, p. 17). These suggestions, though not common practice, provide a useful framework with which to develop more culturally relevant methods of Indigenous knowledge and data protection towards the goal of Indigenous knowledge and data governance.

\subsection{Legal Concerns}

Several scholars have also focused on the unique differences between Western and Indigenous knowledge in the context of intellectual property rights (IPR) and protection. Protection of Indigenous knowledge is recognized by many governments, organizations, and institutions as a timely and critical concern (UNESCO, n.d.). This is increasingly relevant as the application of Indigenous forms of knowledge in Western research is expanding. The Western value in IK has increased significantly in the last decade (Magga, 2009; Anderson, 2015; Bell, 2018) and the National Inuit Research Strategy developed by Inuit Tapiirit Kanatami (ITK) states that "the volume of research within Inuit Nunangat is growing rapidly, but Inuit have little control over it. Between 1996 and 2011, the volume of academic publications related to the four regions of Inuit Nunangat increased by a whopping 200 per cent” (Bell, 2018, para. 10). Thus scholars (Browne, 2012; Engler, Scassa \& Taylor, 2013; Anderson, 2015; Taylor, 2019), national Indigenous organizations (FNIGC, ITK), and the Government of Canada, have identified the need to develop frameworks and protocols for the adequate protection of Indigenous knowledge.

The exploitation of TK is well documented in the literature and clearly demonstrates a need for better protection of Indigenous knowledge and data. TK "has frequently been exploited 
to the detriment of the communities which develop it (Engler, Scassa, \& Taylor, 2013, p. 43) as there is an increasing interest in, and use of, traditional and Indigenous knowledge for commodification whereby communal knowledge and resources are traded in commercial markets that the communities themselves do not take part in (or benefit from). According to Anderson (2015) it is well-known that "many of the new chemicals in medicines have in fact been discovered and developed by Indigenous communities and later taken over, sometimes stolen, and copyrighted by western doctors and companies" (p. 3). Large areas of interest are biological and cultural diversity and environmentalism (Anderson, 2015), two topics which are very likely to be incorporated in the open science domain. This could be problematic as not all traditional/cultural knowledge is necessarily appropriate for sharing. This is especially relevant in the open science context as it requires the use of databases to share and store scientific output. Darrell A. Posey and Graham Dutfield (1996), in their work on intellectual property and resource rights for Indigenous communities, argue that although databases can be protected under copyright law, and users may have to pay for the right to inspect them, "it is difficult for the original suppliers of information in databases to prevent data from entering the public domain and to control use of such information" (p. 28). Browne \& Ljubicic (2019) similarly noted that once research is published in the online domain, it cannot be removed; once consent is given, it is difficult (if not impossible) to retract.

Some have looked to intellectual property/resource rights as a tool for traditional knowledge protection. However, Indigenous people have questioned the legitimacy or benefit of utilizing intellectual property regime (IPR) protection when its mechanisms are antithetical to Indigenous interests: IPR divides knowledge into categories of intellectual, cultural, scientific property, where these elements of knowledge are experienced holistically in Indigenous cultures 
(Posey \& Dutfield, 1996; Anderson, 2015). Consequently, it is widely understood that traditional and Indigenous knowledge is inadequately protected by intellectual property regimes, largely due to their Eurocentric roots.

Research by the Canadian Internet Public Policy Interest Clinic (CIPPIC), in collaboration with the GCRC, may provide some insight about best practices. The Open Licensing Scheme for Traditional Knowledge was developed as part of the Mapping the Legal and Policy Boundaries of Digital Cartography project, with the aim to create a flexible framework to protect Indigenous knowledge using copyleft methods, or Open like licenses and a framework which allows communities and individuals to create their own license based on their individual priorities. Existing intellectual property (IP) laws and traditional knowledge (TK) are incompatible, and TK often "fails to meet substantive requirements for subject matter eligible for copyright protection" (GCRC \& CIPPIC, 2016). Additionally, community-shared knowledge, including knowledge that is shared orally, is not considered as a 'work' by an author under copyright laws. In sum, existing copyright laws are "a poor fit for many forms of TK" (GCRC \& CIPPIC, 2016, p. 4). The GCRC and CIPPIC attempted to address these incompatibilities by developing an open licensing scheme that could prioritize the unique concerns and needs of TK holders, while still utilizing copyright law and contract law to ensure its recognition in the Western legal frameworks such as the Canadian one. Modeled from the copyleft concept, which sees knowledge holders grant the right to distribute and modify intellectual property with the requirement that the same rights be preserved in derivative works created from that property (Wikipedia, n.d.), the scheme offered two different choices for creative commons licenses and 13 labels to add existing protocols for access and use. The licenses included the Attribution-Sharealike, allowing others to change the work so long as they credit the original creator or owner of 
the knowledge and only share under identical terms to the original, and the Attribution-NonCommercial-No-Deriv which only allows users to download the content and share it if the original creator is credited (GCRC \& CIPPIC, 2016). The 13 labels offer potential users the ability to choose which conditions they can add to their content regarding its reuse, which "encompass a much broader set of interests than those contemplated by [Creative Commons] CC licenses" (p. 27). Though the scheme is a proposal and not formally endorsed or by Indigenous communities in Canada, the licensing scheme provides a useful set of best practices for a more culturally relevant knowledge protection process and suggests that alternative mechanisms can be developed to offer traditional knowledge better protection than what is currently offered by contemporary IP laws.

This section described some of the pressing concerns related to sharing TK in online environments when co-management is practiced in research. I will situate my own research within this space, considering the specific ways in which knowledge governance can/cannot be practiced within an OS paradigm, and using these concepts as a portion of my broader analysis of OS and Indigenous knowledge and data governance systems.

\subsection{Summary}

Although the ideals of OS are old, the contemporary OS movement is new, as is Indigenous knowledge and data governance as a recognized practice in Canada, which means that there is little literature that examines the intersection of these two concepts. To address this gap, in Chapter 3 I reviewed literature - including academic journal publications, grey literature, policy documents, reports, and organizational literature - from several domains including science and technology studies (STS), Indigenous and Canadian studies, international development, 
cartography, communication and media studies, public policy, and postcolonial studies to bridge this gap.

Section one reviewed literature which that discussed how Western knowledge production has influenced or impeded the ability of Indigenous nations in Canada to "deconstruct the colonial ideologies of the superiority and privilege of Western thought and approaches" in their Indigenous research (Cull et al., 2018). The authors in focused on the dominance of Western ideology and knowledge that persists in terms of informing institutions, processes, methods, etc. in the sciences. This is important in the context of OS, as discussed, because it brings forward the questions of whether OS is a new form of colonialism and whether it is commensurate with Indigenous data and knowledge governance.

Section two provides some aspects of the historical origin of OS to situate it epistemologically as being driven by the desire to produce more transparent and credible science for the public good. Also, the literature pointed to how scholars in the field generally hold two differing opinions about the OS movement: as some contend that OS will result in a more democratic, collaborative, and transparent field of science, while others argue that OS will further prioritize Western scientific knowledge.

Section three provided a summary of work by scholars who critiqued standards with a postcolonial lens. The authors examined suggested that standards can be postcolonial technologies of power that are inherently political and embody and perpetuate the values of those who design them. This may have negative implications for those whose values are different than the dominant cultural group.

Section four discussed the state of co-development research by examining some examples of this form of research in Canada and summarized some of the benefits and 
challenges this form of research poses. While co-development can result in benefits such as the dissemination and cultural preservation and validation of TK in mainstream science, issues remain over the ability to exercise informed consent, and adequate protection mechanisms for TK and Indigenous knowledge and data.

The semi directed content analysis I conducted and will discuss in the following sections of this thesis, build on this research. These will inform the reading of standards supporting OS and Indigenous knowledge and data governance to consider the ways in which OS and Indigenous knowledge and data governance might align. 


\section{Chapter 4: Methods}

To answer the research questions, I have chosen to conduct a comparative content analysis and case study to better understand the epistemologies, philosophies, and ontologies of Indigenous knowledge and data governance and open science. I will analyze a selection of related academic, grey, and organizational literature to assess the vision, goals, purpose, orientation, and standards in terms of Indigenous data governance and OS and to compare these. The semi-structured content analysis of texts will provide theoretical and applied insight from a range of communities and scholars while the aim of the case study, about the British Columbia First Nations Data Governance Initiative (BC FNDGI), an initiative developed by the First Nations Health Authority (FNHA) and is championed by Elder Gwen Phillips of the Ktunaxa Nation will illustrate Indigenous Data Governances in practice. The aim of the case study is to also illustrate the importance and impact of knowledge governance for First Nations peoples. The BC FNDGI developed one of the first frameworks for data collection and management that prioritizes First Nation knowledge, worldviews, needs, and - most importantly - good 
governance in Canada. This case study is also an example how Indigenous knowledge data governance is related to the realization of sovereignty and self-determination. This will augment knowledge from the content analysis. This case study builds from a one-on-one, semi-structured, exploratory interview with Elder Gwen Phillips.

\subsection{Qualitative Content Analysis}

A content analysis is a method to describe and categorize messages in specific contexts that focuses on the message itself rather than the receivers of the message (Merrigan, Huston \& Johnson, 2012). Content analyses have historically been defined and executed as a quantitative research method, (Bereleson, 1952; Krippendorf, 2004; Merrigan, Huston \& Johnson, 2012; Mcnamara, 2018) however, it is increasingly conducted as a mixed method which draws upon both the quantitative and qualitative. I chose to conduct a qualitative content analysis as this method allows data collected, in this case from texts, to be reduced to "concepts that describe the research phenomenon" (Elo et al., 2014) and "provides the ideal basis for comparative scholarship" (Merrigan, Huston \& Johnson, 2012). My primary goal is to understand the processes and priorities of open science and Indigenous knowledge and data governance separately to determine their theoretical and practical alignment.

\subsection{Humanist vs. Behaviourist}

Pamela J. Shoemaker and Stephen D. Reese (1996), a Communication and a Journalism scholar respectively, contend that there are two traditions of content analysis: the behaviourist tradition and the humanist tradition (Macnamara, 2018). The former is "primarily concerned with the effects that content produces....[and] looks forward from media content to try to identify or predict future effects" (Macnamara, 2018, p. 3). The latter "looks backwards from media content 
to try to identify what it says about society and the culture producing it" (ibid). My analysis will reflect the humanist tradition because the objective of my research is to determine how Western and Indigenous goals, priorities, and objectives are reflected in open science and knowledge and data governance frameworks. Rather than focusing on the effects since my research is concerned with the process of knowledge production and how it is reflective of the societies and cultures in which it is produced. This is an inherently humanist approach (Macnamara, 2018).

\subsection{Inductive vs. Deductive}

Content analysis may also take an inductive or deductive approach (Bereleson, 1952; Krippendorf, 2004; Merrigan, Huston \& Johnson, 2012; Bengtsson, 2016; Mcnamara, 2018). Qualitative content analysis predominantly relies on the inductive approach (Glaser \& Strauss 1967; Strauss \& Corbin 1990; Mcnamara, 2018) which involves "examining the data to identify the topics, issues, and messages that most frequently occur, and then moves back and forth from identifying specifics in texts to making inferences about what those elements might reveal about the speakers, authors, or audience effects" (Macnamara, 2018, p. 205). An inductive approach will be applied here because it is most successful in "cases where there are no previous studies dealing with the phenomenon" (Elo \& Kyngäs, 2008, p. 107) which is the case here as illustrated earlier. Inductive methods are also the most appropriate strategy when there is no pre-existing coding framework, and since inductive content analysis is a more open-ended exploration of data which “creates codes based on the qualitative data itself” (Medelyan, n.d., para. 29). Qualitative content analysis is, therefore, a more flexible method of analysis and has "no specific rules that must be followed" (Bengtsson, 2016, p. 10). This better aligns with the selected literature and topics I am investigating. 


\subsection{Data Collection}

Keeping in mind that there are no specific rules or guidelines to follow during an inductive qualitative content analysis, I will apply a hybrid framework to conduct this research and borrows methods from Elo et al. (2014) and Hall \& Steiner (2020). Elo et al. (2014), who have created a qualitative content analysis framework to prioritize trustworthiness, contend that content analysis processes involve "three main phases: preparation, organization, and reporting of results" (Elo et al., 2014, p. 1). Damon M. Hall and Rebecca Steiner (2020), who are sustainability and environmental scientists, suggest that this necessitates four sets of tasks, three of which will be applied here, and these are: 1) gathering policies within the predetermined time frame; 2) content analysis part 1: identifying the spectrum of policy targets (themes) via qualitative inductive (conventional content analysis) reading of texts; and 3) content analysis part 2: Evaluating the spectrum of themes by qualitative deductive (directed content analysis) comparison to expert recommendations (Hall \& Steiner, 2020).

This framework allows for analytical flexibility, especially regarding the categorization and open coding of themes, key words, and values. This way of doing is also more reflective of a 'native science' practice which goes beyond standardized methodological steps and objective measurement as is popular in Western science (Little Bear, 2000; Cajete, 2000). Instead, a more intuitive approach which is "based on its own conceptual framework" (Cajete, 2000, p. 66) and is flexible according to the community or individual conducting the research.

The first step of content analysis is preparation, which involves the collection of the data and literature to be analyzed (Elo et al., 2014; Hall \& Steiner, 2020). I selected 10 academic, grey, and organizational texts from $2018-2020$ as seen in table 1 below. I used purposive sampling to select these texts where the focus is a single area of study, and not about making 
broad, generalizable conclusions. OS and Indigenous knowledge and data governance are very specific concepts practiced specific ways and purposive sampling allowed for the selection of representative documents to study and upon which to draw conclusions from.

Table 1. Literature for the Content Analysis

\begin{tabular}{|c|c|c|}
\hline Type & Name & Source/Year \\
\hline \multicolumn{3}{|c|}{ Indigenous Knowledge and Data Governance Literature } \\
\hline $\begin{array}{l}\text { Academic } \\
\text { Literature }\end{array}$ & $\begin{array}{l}\text { - CARE Principles for Indigenous Data } \\
\text { Governance }\end{array}$ & Carroll et al., 2020 \\
\hline $\begin{array}{l}\text { Grey } \\
\text { Literature and } \\
\text { Standards }\end{array}$ & $\begin{array}{l}\text { - MUKURTU, digital archive platform by the } \\
\text { Warumungu tribe }\end{array}$ & MUKURTU, 2007 \\
\hline $\begin{array}{l}\text { Organizational } \\
\text { Literature }\end{array}$ & $\begin{array}{l}\text { - National Inuit Strategy on Research } \\
\text { - Principles of Ethical Métis Research } \\
\text { - Ownership, Control, Access, and Possession } \\
\text { (OCAPTM): The Path to First Nations } \\
\text { Information Governance }\end{array}$ & $\begin{array}{l}\text { ITK, } 2018 \\
\text { NAHO, } 2011 \\
\text { First Nations Information } \\
\text { Governance Centre, } 2014\end{array}$ \\
\hline \multicolumn{3}{|c|}{ Open Science Literature } \\
\hline $\begin{array}{l}\text { Grey } \\
\text { Literature }\end{array}$ & $\begin{array}{l}\text { - Open by default and modern, easy to use formats } \\
\text { - 2018-2020 Action Plan on Open Government } \\
\text { - Roadmap for Open Science }\end{array}$ & $\begin{array}{l}\text { Government of Canada, } 2016 \\
\text { Government of Canada, } 2018 \\
\text { Government of Canada, } 2020\end{array}$ \\
\hline $\begin{array}{l}\text { Academic } \\
\text { Literature }\end{array}$ & $\begin{array}{l}\text { - The FAIR Guiding Principles for scientific data } \\
\text { management and stewardship }\end{array}$ & Wilkinson et al., 2016 \\
\hline $\begin{array}{l}\text { Organizational } \\
\text { Literature }\end{array}$ & - Making Open Science a Reality & OECD, 2015 \\
\hline
\end{tabular}

The second phase is organization (Elo et al., 2014). In the inductive approach, the organization phase includes open coding, creating categories, and abstraction (Elo \& Kyngäs, 2008) and identifying the spectrum of themes via qualitative inductive (conventional content analysis) reading of texts (Hall \& Steiner, 2020). Because I will be taking an inductive approach, I will be open coding my texts as I read them. Therefore, I do not have a predetermined list of 
codes, though I will be identifying the topics, issues, and messages that most frequently occur in each set of documents and will be guided by some of the concepts discussed in chapters 2 and 3 of this thesis. Broadly speaking I will be examining the overall goals, objectives, vision, and processes of knowledge and data governance and OS, as well as making inferences about the underlying epistemologies, ontologies, and philosophies of both processes.

Finally, in the reporting phase, "results are described by the content of the categories describing the phenomenon" (Elo et al., 2014, p. 2). Hall \& Steiner (2020) add that this involves evaluating the spectrum of themes by qualitative deductive comparison to expert recommendations. I analyzed the latent content of the selected literature rather than the manifest content. Manifest content would not be in-depth enough for the topic of this research as it requires interpretation rather than primarily counting the presence of my defined units of analysis. Latent analysis is best suited to this project as it can "reveal conceptual frameworks that underpin what is written or said, such as deregulation, privatization, colonization, or technological determinism. Thus, coding of latent content seeks to understand connotation in semiotic terms" (Macnamara, 2018, p. 5). Therefore, documents were analyzed to determine the key words, research areas, theoretical frameworks, research designs and models, data collection tools, participants, variables/research focus and related institutions.

I did not utilize any software to read or analyze the texts. Instead, I read each text line-byline and applied an inductive method, created notes, and identified important parts of the textual data for further analysis. To analyze and compare the findings from each document, I physically color-coded recurring themes and topics and created a chart to visually illustrate where the processes align and where they conflict. These findings are presented in the following chapter. 


\subsection{Semi-Structured Interview \& Case Study}

A key difference between Western and Indigenous science and research processes is that Western processes are often heavily based on theory, whereas traditional approaches rely on and incorporate lived experience in research (Cajete, 2000; Berkes, 2002; Snively \& Williams, 2016). Additionally, oral tradition and stories are frequently used to further understand, relate, and organize information and findings (Snively \& Williams, 2016). Thus, in addition to the content analysis, I also conducted an interview (see Appendix 2) to understand a specific practice of data collection/research that effectively prioritizes Indigenous knowledge and data governance. Where the content analysis provided theoretical knowledge, the interview provided practical knowledge and application of the concepts previously examined. It allowed for a deeper understanding about the importance of Indigenous knowledge and data governance and the impact that it has on Indigenous communities. A few recommendations were made about the congruence of OS and knowledge governance because of this interview.

The interview took place with Elder Gwen Phillips of the Ktunaxa Nation Council. The interview lasted for 1.5 hours over Zoom and included semi-structured (Merrigan, Huston \& Johnson, 2012) questions to better understand the impact, history, and vision of the BC First Nations Data Governance Initiative (BC FNDGI), which was developed by Elder Gwen. I took notes during the interview and recorded the interview, which I then transcribed using the Otter AI live transcript software. To analyze the interview transcript, I open coded to identify major themes and key priorities as described by Elder Gwen. 


\subsection{Researcher Positionality \& Limitations}

Finally, prior to the research process it is important for the researcher to consider "his or her 'pre-understanding', both in the planning process as well as during the analyzing process, in order to minimize any bias of his/her own influence" (Bengtsson, 2016, p.8). As such, I acknowledge that I am a critical data studies scholar who is also a self-identified Ojibwe woman. With this, every effort has been made to ensure that the research design and implementation is free of bias and unaffected, as much as is possible by my positionality.

A perceived limitation of this method is that the results will only reflect an aggregate summary across the literature chosen as a representative sample. While the purpose of this approach is not to pan-Indigenize experiences, histories, and goals, it is limited in that it does not adequately reflect specific local and community protocols that might be developed and used by individual Nations. Another limitation of this study is that there is scant body literature which directly examines Indigenous knowledge and data governance within the OS domain. For this reason, the content analysis will primarily examine grey literature from the Government of Canada, which is guiding the adoption of OS, even though this literature does not specifically address or consider how the process of Indigenous knowledge and data governance can be practiced in tandem. Conversely, the documents (including organizational and academic literature) analyzed about Indigenous knowledge and data governance do not specifically address or acknowledge the practice of OS, or indicate a set of best practices specifically about OS. For this reason, the analysis examines key priorities, practices, and underlying epistemologies of OS and Indigenous knowledge and data governance separately and compares them to determine where they might align and diverge. In sum, a research limitation here is the lack of substantive literature addressing both these concepts together. 


\section{Chapter 5: Observations and Analysis}

\subsection{Introduction}

Section 5.2 of this chapter provides a structured analysis of the supporting literature and case study. In this section I look for the vision, goals, purpose, and orientation of OS and Indigenous knowledge and data governance as they are articulated in their standards, organizational and grey literature. I also identify the underlying epistemology, ontology, and philosophy of each paradigm. This includes an analysis of the interview with Elder Gwen Phillips, whose professional insight on the effective governance of Indigenous data is used to demonstrate a specific case study of First Nations data governance which offers insight about its impact and goals. This section is presented concept-by-concept for clarity.

Section 5.3 will show the findings resulting from both processes and an assessment of how they might align and misalign, and this is ascertained from the previous summary. This section makes connections between OS and Indigenous knowledge and data governance to 
identify where the goals, vision, priorities, and objectives are theoretically and practically similar, and where they are fundamentally incommensurable. This section also acknowledges the ways in which specific components are neither aligned nor misaligned, and where they can theoretically function together. However, it is important to acknowledge that, just because the concepts do not misalign, it does not mean the processes are mutually beneficial. In other words, just because there are aspects of OS that are not fundamentally misaligned with the current standards and conceptions of OS in Canada, it does not mean that OS, as it is currently articulated, is beneficial and/or relevant for First Nations, Métis, or Inuit communities. This will be further elaborated in the succeeding sections.

\subsection{Observations}

\subsection{Open Science}

To better understand the major goals, vision, orientation, and purpose of OS I analyzed 5 key documents (See Table 1 above). The publication Making Open Science a Reality is a public policy paper by the OECD Science, Technology, and Industry sector and provides a foundational overview of the process of OS and its implementation using evidence-based recommendations and observations. This is also relevant because Canada is a member of the OECD and is therefore influenced by the policy directives and recommendations that come out of this organization. To further understand the specific implementation of OS in Canada, I analyzed The Roadmap for Open Science, the 2018-2020 National Action Plan on Open Government, and the Open by default and modern, easy to use formats from the Government of Canada. The impetus for the OS roadmap was a recommendation in the Open Government Action Plan, mandating the adoption of OS that led to the Roadmap as a strategic outcome. Open by default is a policy 
directive to implement OS as well as open government and open data initiatives in Canada more broadly. Finally, The FAIR Guiding Principles for scientific data management and stewardship was analyzed because the FAIR principles are a leading de-facto standard for open research data sharing globally, and are a recommendation in Canada's Open Science Action Plan. This publication I examined was the first formal publication of the FAIR Principles.

Taken together, these documents articulate a very specific vision, set of goals, orientation, and purpose for OS in the global and Canadian context. Their underlying philosophy, epistemology, and ontology are also evident in these documents.

\section{Vision}

The vision for OS as articulated in each of the documents analyzed refers to their overarching plan: the accomplishments that are guiding the implementation of OS. In some cases, the vision was explicitly stated, and in others, the vision was indirectly stated as the end goal for OS. Both versions were recorded and coded.

The vision for OS is relatively the same in the global and national context, and as articulated in the OECD policy paper and Government of Canada's mandates. The OECD policy paper states that the vision for OS is a world in which scientific output, research findings and related data, and scholarly publications are publicly accessible in digital format with little or no restriction to maximize the effectiveness and productivity of federally funded scientific research (OECD, 2015). OS aims for a complete transformation of the scientific environment, including the way science is performed, disseminated, and managed (OECD, 2015). This vision can be implemented with the support of policies, licenses, and standards. 
This is consistent with the vision articulated by the Government of Canada, which indicates that OS will "accelerate progress towards solving society's most persistent problems" and is a "catalyst for change in the way we practice science, how we create opportunities for innovation, and in how we build new partnerships in industry" (Government of Canada, 2020, para. 7). Specifically, the Roadmap for Open Science states that its vision is to "make Canadian science and research open to all, maximizing benefits for the well-being, health, and economy of our country" (Government of Canada, 2020, "Vision" para.1). The roadmap indicates that, to achieve this vision, scientific and research output should be "as open as possible". The vision for Canadian OS is consistent in the Roadmap and the Open Government Action Plan, which envisions effective federal openness as a complete shift in governing culture - including capabilities, behaviors, and processes - which would result in greater openness, accountability, and reduced cost by maximizing reusability of data (Government of Canada, 2018).

The impetus for the FAIR principles, which stands for Findable, Accessible, Interoperable, and Reusable, are consistent with this vision. The FAIR principles stipulate that all digital objects should be findable, accessible, interoperable, and reusable to facilitate increased innovation and knowledge discovery through standardized data management practices (Wilkinson et al., 2016). The principles are a set of guidelines that map onto the OS process as one of the primary tools for open data sharing of research that is globally recognized and implemented (Wilkinson et al., 2016). The vision of open research and science data, as articulated in both the principles and their foundational literature, is for "all digital objects of all kinds to become first class citizens in the scientific publication ecosystem, where quality of publication... is a function of its ability to be accurately and appropriately found, reused, and cited" (Wilkinson et al., 2016, p. 1). Like the vision of OS, the primary vision of the FAIR 
principles is for openness in the science and research domain as a catalyst for methodological and institutional transformation.

\section{Goals \& Objectives}

The goals and objectives of OS are consistent throughout the documents analyzed. The primary goals of OS articulated in the Open Science Roadmap are: ensuring accountability, increasing reproducibility, creating open engagement, reducing duplication, creating opportunities for impact, leveraging diversity and inclusion, accelerating knowledge transfer, and building synergies with international and domestic OS movements (Government of Canada, 2020, "Why open science"). The objectives proposed to achieve these goals include the following:

- increased consultation with internal and external partners in the scientific community to address benefits and challenges of the OS action plan;

- making federal science articles and publications openly accessible by January 2022-2023;

- implementing the FAIR principles to ensure interoperability of research data and metadata;

- enabling the open by design and by default model for scientific research outputs of scientific projects and research;

- development of a framework identifying criteria when restricting access to federal scientific research outputs is warranted;

- and the development of an OS action plan for research conducted outside of federal government agencies and departments, in collaboration with federal granting agencies (Government of Canada, 2020).

The open by default and easy to use formats publication aspects reiterates the goal of open by default (OBD) which is to "make government data and information open by default, in formats that are modern and easy to use" (Government of Canada, 2016, para. 1).

Similarly, the objectives of openness are articulated in the Open Government Action Plan rest on three primary goals: transparency, accountability, and citizen participation (Government of Canada, 2018). The described objectives to achieve these goals include: 
- increasing the reusability of government data;

- facilitating a more democratic and participatory climate in government and science;

- increasing accountability of scientific and governmental institutions and bodies (Government of Canada, 2018).

The key objectives of the FAIR principles are:

- encouraging researchers and scientists to practice good data management and stewardship through long-term care of digital assets;

- developing high quality digital publications that facilitate and simplify processes of discovery, evaluation, and reuse in subsequent research; and

- increasing the ability of humans and machines in the discovery of, access to, and integration and analysis of task-appropriate scientific and research data (Wilkinson et al, 2016).

There are six primary goals of OS that are articulated in the OECD policy paper. Open science according to the OECD aims to improve scientific and research processes by:

1. Reducing duplication costs in collecting, creating, transferring, and reusing data;

2. allowing more research from the same data;

3. multiplying opportunities for domestic and global participation in the research process;

4. creating the conditions for greater scrutiny of research results, which will result in more accurate verification (and reproducibility) of results;

5. closer involvement and participation of citizens; and

6. promotion of a broader understanding of science, evidence-based practices, and citizen science (OECD, 2015).

The goals and objectives of OS articulated in these documents overlap and can be categorized into two major groups: people-centered goals and data-centered goals, for example, people-centered goals would include closer involvement and participation of citizens and increased citizen science initiatives, while data-centered goals refer to reducing duplication costs in collecting, creating, transferring, and reusing data, and making data easier to find so it can be reused. 


\section{Purpose}

The Government of Canada Action Plan on Open Government states that the purpose for OS is that, while "public access to science conducted or collected by the federal government has great potential value... government-funded science is sometimes hard to access" (Government of Canada, 2020, section 5) as it is not open, easy to find, or effectively communicated to the public (ibid). Subsequently, the Roadmap for Open Science states that the primary purpose of OS is to make scientific information accessible to all. The purpose of making scientific research outputs publicly accessible is to ensure accountability, increased reproducibility, create open engagement, and reduce duplication of research for more efficient and economical science (Government of Canada, 2020). The purpose of sharing open data as it is articulated in the Open by default and modern, easy to use formats policy directive is "revitalizing access to information" that can be valuable to Canadians (Government of Canada, 2016, "Interim Policy Guidance", para. 1).

As for the FAIR principles their purpose was to address the "urgent need to improve the infrastructure supporting the reuse of scholarly data" (Wilkinson et al., 2016, p. 1). The FAIR principles are also meant to facilitate good data management and lead to increased knowledge discovery and innovation, as well as increased reuse of data and research after they are published (Wilkinson et al., 2016).

Finally, the OECD policy paper states that the purpose of OS is part of the means to address 21 st century science issues. The paper suggests explains that "economists consider scientific knowledge generated by public research as a public good, which means that everyone can make use of that knowledge at no additional cost once it is made public, generating higher social returns" (OECD, 2015, p. 7). In addition, OS is to enable "greater access to scientific 
inputs and outputs [that] can improve the effectiveness and productivity of the scientific and research system" (OECD, 2015, p.10) by reducing duplication costs, enabling greater reuse of the same data, allowing the greater scrutiny of scientific research for more credible scientific output, and increasing general access to publications and research data (OECD, 2015).

In sum, the overall purpose of OS as articulated in the literature examined here is to address the inaccessibility, exclusivity, and irreproducibility of national government funded scientific output. Contemporary scientific and publication environments have presented barriers to the free flow of relevant, timely, accurate, and accessible knowledge and information for the public; where these are shared in a way that makes them exclusive and hinders public engagement; and are frequently managed poorly, which contributes to their limited reuse. The purpose of OS is therefore to rectify the issues with modern science processes and to restore credibility, engagement, and good data management, which includes the findability, accessibility, interoperability, and reusability (FAIR) of scientific research data (Government of Canada, 2018, 2020; OECD, 2015; Wilkinson et al., 2016).

\section{Orientation}

Open science is both a human and data-centered initiative. The mechanisms by which OS is theorized, implemented, and practiced are largely data centric whereby the FAIR principles are a technical guideline to enhance the findability and reusability of data and metadata, and open by default is a technical specification which would require research to be made open upon publication. However, OS is also rooted in the concept of knowledge as a public good. The primary rationale for the OS movement is to increase social and economic benefit related to scientific endeavors: making scientific research outputs more accessible to the public, as 
indicated above, would provide citizens with the ability to make use of publicly funded research at no additional cost once it's made public, generating both higher social returns and allowing more research from the same data, thereby reducing costs, avoiding duplication and redundancy. This is clearly iterated in the OECD policy paper which presents OS as a data-centered process to make outputs open, with tangible people-centered benefits (OECD, 2015).

The OS Roadmap is both people and data oriented, though there is more emphasis on the social benefits of OS for Canadian citizens, researchers, and scientists. While the Roadmap includes data-centered principles like the implementation of FAIR principles and open-bydefault specifications, the Roadmap makes clear that the primary benefits of an OS approach are people-centered and the move to open the scientific environment will result in increased democracy, transparency, and innovation (Government of Canada, 2020). The open-by-default $(\mathrm{ObD})$ policy paper indicates that $\mathrm{ObD}$ is a data-centered policy to maximize openness and transparency (Government of Canada, 2016).

The FAIR principles are a data-centric approach to good data management and stewardship, and the guidelines are "distinct from peer initiatives that focus on the human scholar" (Wilkinson et al., 2016, p. 1) as they distinctly focus on "enhancing the ability of machines to automatically find and use the data, in addition to supporting its reuse by individuals" (ibid).

In sum, the process of open science is equally people and data oriented. The processes by which OS is implemented, practiced, and sustained are primarily data-centered, and focus on the requirements necessary to make research and scientific data/outputs freely available with little to no restriction. This includes (meta)data standards like the FAIR principles, and the requirement to make data $\mathrm{ObD}$ in open formats and repositories. 


\section{Philosophical Investigation}

The fundamental concept of knowledge as a public good is at the heart of OS (OECD, 2015; Government of Canada, 2020). The knowledge commons ideology posits that sharing knowledge leads to increased economic and social benefit. This is embodied in the methods, goals, values, and standards of OS, which considers scientific knowledge as a public good that should be made available with as few restrictions as possible.

Epistemology is the branch of philosophy that investigates how we create knowledge. The epistemological underpinning of open science is objectivism, which posits that "all human knowledge is reached through reason" (Thomas, 2010, para. 1). As an extension of 'modern' science which employs rationalism, the philosophical ideology that knowledge comes from rational thought (Thomas, 2010), OS does not deviate from this philosophical underpinning and is epistemologically the same.

Ontology, or the study of being, is concerned with what exists in the world and how humans can acquire knowledge (Moon \& Blackman, 2017). The ontological orientation of science is realism; Western science presumes that reality - the single, objective reality - can be understood using appropriate methods which should produce knowledge, the validity of which should be "defended on moral grounds" using the scientific method (Maturana, 1990, p. 12) as Western science considers methods superior to all others (Hickey, 2020, p. 14). Again, OS does not deviate from this philosophical underpinning as the scientific process itself remains largely unchanged.

\subsection{Indigenous Knowledge and Data Governance}

Indigenous knowledge and data governance (IKDG) is a nuanced and dynamic concept that does not have one fixed definition or process of implementation. There are different visions, 
goals, and purposes that reflect the needs and priorities of different Indigenous communities. I have identified the broad concepts articulated in the literature I selected to analyzed in to better compare with OS. However, it is important to note that the goal of this analysis is not to panIndigenize experiences, objectives, motivations, or histories. For that reason, this analysis is not exhaustive nor definitive: my findings are a consensus from a representative sample of selected literature that could not comprehensively or holistically address the breadth of perspectives, worldviews, and voices from all peoples. I also acknowledge that there are nuances that cannot be simply summarized, analyzed, and empirically compared. Most significantly, each of the concepts I have chosen to analyze are intended to govern different types of knowledge and data. The following is a summary of each concept as it is articulated in the publicly accessible research ethics guides and principles from the First Nations, Inuit, and Métis organizations I examined.

I analyzed five documents to study their visions, goals, objectives, purpose, and philosophy of Indigenous knowledge and data governance as it is articulated, directly or indirectly, by First Nations, Métis, and Inuit organizations in Canada. The Principles of Ethical Métis Research, a document produced by the National Aboriginal History Organization (NAHO) and Métis communities, presents one of the only Métis-specific publications for research involving Métis people and/or knowledge/information, highlighting Métis-specific expectations and priorities. The Inuit Tapiirit Kanatami's National Inuit Strategy on Research provides a wealth of information about Inuit expectations, priorities, and needs as they pertain to governance and self-determination in research and science. The CARE Principles for Indigenous Data Governance are a set of principles developed by Research Data Alliance (RDA) International Indigenous Data Sovereignty Interest Group in collaboration with Indigenous organizations to address the lack of Indigenous-specific consideration in the FAIR principles as 
it pertains to data governance. Each of these three sets of principles are intended to govern Indigenous knowledge and data, primarily as they pertain to research data. Ownership, Control, Access and Possession (OCAP): The Path to First Nations Information Governance written by the First Nations Information Governance Centre discusses the necessary requirements for First Nations peoples to exercise jurisdiction over their own data, as embodied in the OCAP principles. Though the principles were initially developed to assert First Nations governance over health data, the principles are increasingly applied to understand the implications of open data in the OS context for First Nations information, knowledge, and data more broadly. Finally, MUKURTU is an online content management system (CMS) developed by the Warumungu tribe to exercise cultural management and preservation in the online domain, specifically as it pertains to cultural artefacts, art, and historical knowledge about these artefacts. MUKURTU is a repository and content management system for Indigenous cultural artifacts and to share and preserve borne digital and digitized content in culturally defined. It is examined here because it is an online repository open according to Indigenous permissions value sets.

\section{Vision}

As mentioned previously, it is not possible to state a collective vision for Indigenous knowledge and data governance due to its inherent ontological characteristics: the conception of First Nations information governance is not the same for every FN community, let alone representative of Métis or Inuit conceptions of knowledge and data governance nor of traditional knowledge. The vision of knowledge and data governance, as articulated in the documents selected here will be analyzed. 


\section{i. Principles of Ethical Métis Research}

The Principles of Ethical Métis Research's vision for effective Métis research governance is for it to be conducted in an environment in which research is developed collaboratively with Métis peoples; where the incorporation of Métis specific principles and ethics are included in research design, and research includes goals that are "consistent with the needs and unique cultural perspective of Métis" (NAHO, 2011, para. 2). The principles taken together offer a holistic representation of Métis specific expectations in research; the underpinning of these principles is the necessity of Métis governance and influence in the research process including decision-making, agenda-setting, data stewardship, and benefit sharing, where research is "practiced in a way where Métis people are consulted and involved in the research" (NAHO, 2011, para. 2).

\section{ii. CARE Principles}

The vision of Indigenous information governance articulated in the CARE Principles is to "reposition Indigenous Peoples, nations, and communities from being subjects of data that perpetuate unequal power distributions to self-determining users of data for development and wellbeing" (Carroll et al., 2020, p.2). Furthermore, the vision is that "Indigenous Peoples have continuity with their pre-colonial societies and [are able] to use their own social, political, and economic systems to preserve, develop, and transmit to future generations their cultures [and] knowledges (Martinez Cobo 1982 in Carroll et al., 2011, p. 2). This necessitates the meaningful "incorporation of Indigenous knowledge and approaches into data and research practices and policies" (Carroll et al, 2011, p. 1). 


\section{iii. National Inuit Strategy on Research}

The vision of governance articulated in the National Inuit Strategy on Research by ITK is a transformation of institutions (research governance bodies, governments), methods, and set of policies to respect and support Inuit self-determination in research related to 1) Inuit peoples and/or 2) the Inuit Nunangat homeland, and where Canadian Inuit are prospering through unity and self-determination (ITK, 2018, "Vision").

\section{iv. OCAP Principles}

OCAP principles, which stands for ownership, control, access, and possession of Indigenous data for Indigenous data governance, are driven by the vision of restoring First Nation jurisdiction of data, knowledge, and information pertaining to them (FNIGC, 2014). The principles are a flexible framework which, ideally, are meant to ensure that First Nation data are under the jurisdiction of the people who provide them or data about them or data managed by them, enabling First Nation individuals and communities to exert governance over their own data. Through effective knowledge governance, this is one way toward archiving selfdetermination and sovereignty. "OCAP ${ }^{\circledR}$ is a vision and protocol for principled research that protects First Nation people and information from harmful, insensitive, and exclusive research relationships" (FNIGC, n.d., "OCAP FAQ", para. 13).

\section{v. MUKURTU}

Finally, MUKURTU is an example of a standards-based content management system (CMS) for Indigenous borne digital, digitized and the cataloguing of artifacts. It is rooted in the principle of Indigenous knowledge and data governance in the art, culture, and museum sectors. The vision is to empower Indigenous communities to exercise data and knowledge governance of curated artifacts and artistic endeavors online in the same way that it is 
exercised in face-to-face or when viewing artifacts in person. The vision of knowledge governance articulated here is to restore control over cultural artifacts and their dissemination - including data, metadata, and classification systems - to Indigenous peoples to facilitate selfdetermination through cultural management and knowledge preservation and dissemination.

\section{Goals}

\section{i. Principles of Ethical Métis Research}

In the literature for the Principles of Ethical Métis Research, the primary goals include: the development and maintenance of reciprocal relationships between researchers and communities with shared responsibilities and benefits; recognition of diversity within and between Métis peoples; respect for Métis peoples, autonomy, identity, personal values, gender, confidentiality, practices and protocols; safe and inclusive environments for all peoples involved; as well as research that is relevant, beneficial for all, accurate, accountable, that acknowledges contributions equally and protects Métis cultural knowledge; and recognizes Métis historical context including Métis values and knowledge, methodologies, and diverse worldviews (NAHO, 2011).

\section{ii. CARE Principles}

The goals of knowledge governance as articulated in the CARE principles are: a repositioning of Indigenous peoples from subjects of data to self-determining users of data for development and well-being; divesting colonial values, lifestyles, and suppression of Indigenous knowledge and data systems; to recover, develop, and sustain Indigenous knowledge; the advancement of self-determination and reclamation of cultural identity; more equitable outcomes for Indigenous peoples in the scientific and research domain; and the strengthening of Indigenous control, access, reuse, and discovery of data (Carroll et al., 2020, p. 2). 
Governance also includes incorporating Indigenous priorities as they relate to science, research, and data collection; data should be used for improved governance and engagement and inclusive development and innovation, and research should be reflective of community values (Carroll et al., 2011).

\section{iii. National Inuit Strategy on Research}

The goals of knowledge governance articulated in the ITK strategy are: improving methods for sharing data with Inuit peoples in effective ways; developing data strategy parameters to facilitate Inuit and community control over the collection, interpretation and ownership of Indigenous data; the development of "processes, protocols, standards, and agreements that allow for the safe sharing of certain information"; and "the respectful incorporation of Inuit knowledge in data management and sharing design and implementation" (ITK, 2018, p. 21). Perhaps most importantly, self-determination is the overarching goal of governance in research contexts involving Inuit peoples and/or their knowledge and data.

\section{vi. OCAP Principles}

The goals of OCAP are: to provide First Nation with the tools to exercise jurisdiction over their information and data through proper control, ownership, access, and possession; to ensure that First Nations information and data are used in a way that brings benefit to the community and minimizes harm; to divest colonial relations and approaches to knowledge production; to restore First Nations control of data, knowledge and information (in all contexts); to exert governance over data held by 'outsiders' through repatriation of data, or with data sharing agreements to restore First Nations control; to foster recognition of the context and history of First Nations communities and individuals in research; and to aid in the restoration of sovereignty (FNIGC, 2014). 


\section{vii. MUKURTU}

MUKURTU content management system allows its community of users to exercise governance over their cultural knowledge and information. The standards and protocols indicate the goals and priorities of effective knowledge governance which include permissionbased sharing and preservation of cultural knowledge, digital stewardship, preservation, access, and sustainability of traditional knowledge, and control of decision-making processes over parameters of sharing knowledge (MUKURTU, n.d., "About”).

\section{Purpose}

i. $\quad$ Principles of Ethical Métis Research

The purpose of Métis-specific principles (which support the Métis governance of research) is because "there is very little evidence or documentation around the use of Métis specific research ethical guidelines or principles" (NAHO, 2011, para. 2). Furthermore, Métis interests, perspectives, priorities, and research needs have seldom been included in, or helped guide, research about or with Métis peoples (NAHO, 2011). While the principles are not meant to be a set of enforceable rules that must be strictly followed, they are described as "a well thought out starting point to engage Métis communities in ethical research" (NAHO, 2011, para. 3) in alignment with the goal of data and knowledge governance.

\section{ii. CARE Principles}

The CARE principles for Indigenous data governance were developed because "the current movement toward open data and open science does not fully engage with Indigenous Peoples' rights and interests" (GIDA, 2020) and "mainstream values related to research and data are often inconsistent with Indigenous cultures and collective rights" (Carroll et al., 2020, p. 3). The goal of the principles is to advance the recognition and effective exercise of Indigenous 
data governance and self-determination; as such, the purpose articulated in these principles is to reclaim the inherent right to self-determination in research, which includes the right to exercise control over the data about "their peoples, communities, cultures, and territories" (Carroll et al., 2020, p.1). Finally, the purpose of knowledge and data governance is that "Indigenous Peoples must have access to data that support Indigenous governance and selfdetermination...[and] be the ones to determine data governance protocols, while being actively involved in stewardship decisions for Indigenous data that are held by other entities (Carroll et al, 2020, p. 6).

\section{iii. National Inuit Strategy on Research}

The purpose of knowledge governance articulated in the ITK National Inuit Strategy on Research is to counter the historical and current state of research in Canada, which continues to embody settler colonial legacies and methods. The strategy indicates that "colonial approaches to research endure in Canada that prevent Inuit from making decisions about research activity in our homeland, such as setting the research agenda, monitoring compliance with guidelines for ethical research, and determining how data and information about our people, wildlife, and environment is collected, stored, used, and shared" (ITK, 2018, p. 4). The purpose of knowledge and research governance is to protect and advance the rights and interests of Inuit in Canada (ITK, 2018).

\section{viii. OCAP Principles}

The OCAP principles offer a First Nations-centered approach to ethical research and information and data management. The literature asserts that "in the past, Aboriginal (sic) people have not been consulted about what information should be collected, who should gather that information, who should maintain it, and who should have access to it" (FNIGC, 
2014, p. 5). In addition, "information gathered may or may not have been relevant to the questions, priorities and concerns of Aboriginal (sic) peoples" (ibid) and this struggle persists. Therefore, the purpose of OCAP - as a mechanism of First Nations governance - is to ensure that contracts, agreements, licenses to use and other forms of legal agreement whereby First Nation data and information is shared, contains the necessary provisions to protect First Nations ownership, control, access, and possession of all First Nations data. (FNIGC, 2014, p. 21) The purpose of effective governance, and OCAP by proxy, is to advance selfdetermination and data sovereignty.

\section{ix. MUKURTU}

The purpose of MUKURTU, as an exercise of governance, is to enable effective Indigenous cultural management and preservation. With cultural protocols, traditional knowledge labels, and community records, MUKURTU enables Indigenous peoples to exercise fine-grain control over their own digital cultural heritage. The purpose of the CMS is to provide a safekeeping place for cultural heritage in digital formats; the system itself is a mechanism of information governance, the purpose of which is to protect and share knowledge according to culturally specific interests.

\section{Orientation}

Indigenous knowledge and data governance is oriented towards First Nations, Métis, and Inuit peoples. The visions, goals, objectives, and purposes as articulated in the literature indicate that knowledge governance is, first and foremost, meant to restore control to Indigenous peoples to exercise jurisdiction over their knowledge, data, and information. Governance is articulated as a process through which self-determination and sovereignty can be realized. Governance, selfdetermination, and sovereignty are central to identity and nation rebuilding for Indigenous 
peoples which are cultural determinants of health and wellbeing (FNIGC, 2014; ITK, 2018; Phillips, 2017).

\section{Philosophical Investigation}

Epistemology: Broadly speaking, and based on these readings, Indigenous peoples collectively share a world view that differs from non-Indigenous Canadians and, and how knowledge is created and thought of is also different. Indigenous and non-Indigenous peoples therefore have different epistemologies. Epistemology is the branch of philosophy that aims to understand how we create knowledge (Moon \& Blackman, 2017) and helps to explain how different people relate to the world with which they engage. Indigenous epistemology cannot simply be reduced to one concept and in one context within which it operates: Indigenous epistemology underpins every aspect of Indigeneity and frames worldviews, values, and knowledge. Indigenous epistemology, like Indigenous ontology, is more holistic and relational in contrast to the empiricist and rationalist ontologies of western science.

Ontology is concerned with what exists in the world that we can possibly know about, and Indigenous ontology suggests that there are many ways of producing knowledge and that all knowledge and methods of research are rooted within a particular cultural orientation which in turn influences the knowledge that is produced. Whereas Western science considers the scientific method to be the best and most effective way to uncover objective truth about the natural world, Indigenous ontology necessarily understands that there can be no truly objective account of natural phenomena, but that does not mean that scientific approaches are dismissed. An Indigenous ontology suggests that "all knowledge originates in people's culture - method of research always stems from a cultural orientation, a paradigm of thinking that has a history in 
some particular tradition. Therefore, there can be no such thing as a fully objective story of the universe" (Cajete, 2004, p. 46).

\subsection{Comparative Analysis}

In this section I will compare the above-described elements of open science and Indigenous knowledge and data governance. Table 3 below is a summative, side-by-side comparison of each of the analyzed elements of open science and Indigenous knowledge and data governance while Table 4 illustrates the major areas of alignment and misalignment between each process. While Table 3 provides a concise summary of components analyzed and described in the above section, Table 4 demonstrates the key similarities and differences between the processes and will be the basis for discussion in the comparative analysis to follow.

Table 2. Open Science vs. Indigenous Knowledge and Data Governance Summary

\begin{tabular}{|c|c|c|}
\hline & Open Science & $\begin{array}{c}\text { Indige nous knowledge and data } \\
\text { governance }\end{array}$ \\
\hline Vision & $\begin{array}{l}\text { A transformation of the scientific } \\
\text { environment - including how science is } \\
\text { done, critiqued, shared, and reused - to } \\
\text { ensure maximum benefit. }\end{array}$ & $\begin{array}{l}\text { A restoration of Indigenous governance over } \\
\text { knowledge, data, information, and research } \\
\text { pertaining to Indigenous peoples towards the } \\
\text { increased well-being, nation rebuilding, and } \\
\text { cultural restoration of Indigenous peoples. }\end{array}$ \\
\hline $\begin{array}{l}\text { Goals \& } \\
\text { Objectives }\end{array}$ & $\begin{array}{l}\text { - To make research and science as open as } \\
\text { possible with minimal to no restriction. } \\
\text { - To promote increased citizen engagement, } \\
\text { participation, and democracy. } \\
\text { - To increase scientific innovation, reuse of } \\
\text { research, maximize knowledge from } \\
\text { research outputs, and produce more } \\
\text { credible and accurate science. }\end{array}$ & $\begin{array}{l}\text { - Indigenous assertion of ownership over } \\
\text { their own identity through governance of } \\
\text { knowledge and data. } \\
\text { - Advancement of self-determination and } \\
\text { sovereignty. } \\
\text { - A repositioning of Indigenous peoples } \\
\text { from subjects of data to self-determining } \\
\text { users of data for development and well- } \\
\text { being. }\end{array}$ \\
\hline Purpose & $\begin{array}{l}\text { The purpose of making scientific research } \\
\text { outputs publicly accessible is to ensure } \\
\text { accountability, increased reproducibility, } \\
\text { create open engagement, and reduce } \\
\text { duplication of research for more efficient } \\
\text { and economical science. This is in response }\end{array}$ & $\begin{array}{l}\text { The purpose of Indigenous knowledge and } \\
\text { data governance is to counter historical } \\
\text { colonialism in research, data and knowledge } \\
\text { collection and dissemination. Indigenous } \\
\text { peoples in Canada have long suffered from } \\
\text { colonialism in research and have been }\end{array}$ \\
\hline
\end{tabular}




\begin{tabular}{|c|c|c|}
\hline & Open Science & $\begin{array}{l}\text { Indigenous knowledge and data } \\
\text { governance }\end{array}$ \\
\hline & $\begin{array}{l}\text { to common issues with modern science } \\
\text { including irreproducibility, lack of } \\
\text { credibility, and closed scientific research } \\
\text { that is not accessible en masse. }\end{array}$ & $\begin{array}{l}\text { research subjects, subject to knowledge and } \\
\text { data extraction and exploitation, and have not } \\
\text { been recognized as rightful owners to their } \\
\text { own data. The purpose of Indigenous } \\
\text { knowledge and data governance is to counter } \\
\text { this colonial history and restore ownership } \\
\text { and decision-making rights to the people who } \\
\text { rightfully own the data and knowledge. }\end{array}$ \\
\hline Orientation & People and data-centered & People centered \\
\hline Philosophy & $\begin{array}{l}\text { Knowledge commons - science should be } \\
\text { available to as many as possible, knowledge } \\
\text { is a common good. }\end{array}$ & $\begin{array}{l}\text { Relational - everything is related, holistic, } \\
\text { subjective. There is no clear division between } \\
\text { different fields of knowledge: all forms of } \\
\text { knowledge and ways of knowing are } \\
\text { interrelated. }\end{array}$ \\
\hline Epistemology & $\begin{array}{l}\text { Knowledge is produced by carrying out } \\
\text { objective science to uncover objective truth. }\end{array}$ & $\begin{array}{l}\text { There are many kinds of knowledge that exist } \\
\text { and there are various ways to produce and } \\
\text { interpret them. }\end{array}$ \\
\hline Ontology & $\begin{array}{l}\text { Realism: The single, objective reality can be } \\
\text { understood using appropriate methods } \\
\text { which should produce knowledge, the } \\
\text { validity of which should be "defended on } \\
\text { moral grounds" using the scientific method. }\end{array}$ & $\begin{array}{l}\text { Truth is ever evolving and is subjective to } \\
\text { researcher positionality. There is no such } \\
\text { thing as complete objectivity. }\end{array}$ \\
\hline $\begin{array}{l}\text { Who produces } \\
\text { knowledge? For } \\
\text { whom? }\end{array}$ & Researchers and scientists for the public. & $\begin{array}{l}\text { - Knowledge should be produced with } \\
\text { Indigenous peoples through co- } \\
\text { development practices and should always } \\
\text { be reciprocal. } \\
\text { - Research done by Indigenous peoples is } \\
\text { done for the benefit of the community and } \\
\text { collective benefit to know more about } \\
\text { themselves. }\end{array}$ \\
\hline Governance & $\begin{array}{l}\text { - OS research, data, output is made open by } \\
\text { default, and shared in open repositories } \\
\text { with open licenses Governance of } \\
\text { research output is unclear: who retains } \\
\text { ownership? } \\
\text { - Research would be in the possession of } \\
\text { the Government of Canada. }\end{array}$ & $\begin{array}{l}\text { - Knowledge and data belong to the } \\
\text { community (inherent right) } \\
\text { - Each individual nation has the right to } \\
\text { exercise control and governance of their } \\
\text { own data, knowledge: this includes } \\
\text { research that is informed by IK as well as } \\
\text { data about Indigenous Peoples. }\end{array}$ \\
\hline $\begin{array}{l}\text { Methods of } \\
\text { application }\end{array}$ & $\begin{array}{l}\text { - IP } \\
\text { - Data repositories } \\
\text { - Standards }\end{array}$ & $\begin{array}{l}\text { - Community standards and principles } \\
\text { - UNDRIP } \\
\text { - Data sharing agreements }\end{array}$ \\
\hline Standards & $\begin{array}{l}\text { - FAIR } \\
\text { - Open by default }\end{array}$ & $\begin{array}{r}\text { - CARE } \\
\text { OCAP }\end{array}$ \\
\hline
\end{tabular}




\begin{tabular}{|l|l|l|}
\hline & \multicolumn{1}{|c|}{ Open Science } & \multicolumn{1}{c|}{$\begin{array}{c}\text { Indigenous knowledge and data } \\
\text { governance }\end{array}$} \\
\hline & & $\begin{array}{l}\text { - MUKURTU example: traditional } \\
\text { knowledge, cultural protocols }\end{array}$ \\
\hline $\begin{array}{l}\text { Is it considered } \\
\text { in federal } \\
\text { mandates for } \\
\text { open science? }\end{array}$ & Yes & No \\
\hline
\end{tabular}

Table 3. Open Science vs. Indigenous Knowledge and Data Governance: Commonalities \& Differences

\begin{tabular}{|l|l|}
\hline \multicolumn{1}{|c|}{ Commonalities } & \multicolumn{1}{c|}{ Differences } \\
\hline $\begin{array}{l}\text { Philosophy: Both processes are meant for "social } \\
\text { benefit" }\end{array}$ & $\begin{array}{l}\text { Governance: Indigenous governance of related knowledge } \\
\text { and data versus open governance of all research output }\end{array}$ \\
\hline $\begin{array}{l}\text { Goal: Both processes are meant to maximize the } \\
\text { benefit of data and reduce harm }\end{array}$ & $\begin{array}{l}\text { Goal: Making scientific and research output open by default } \\
\text { and design versus OCAP }\end{array}$ \\
\hline $\begin{array}{l}\text { Goal: Both processes aim to collect quality, } \\
\text { accurate, and relevant data }\end{array}$ & $\begin{array}{l}\text { Goal: A key motivation for adopting OS is to maximize } \\
\text { efficiency, reduce duplication of research efforts (for } \\
\text { financial reasons) }\end{array}$ \\
\hline $\begin{array}{l}\text { Goal: Increased citizen participation and } \\
\text { engagement }\end{array}$ & $\begin{array}{l}\text { Purpose: advancing self-determination in research and } \\
\text { knowledge governance versus to make scientific research } \\
\text { output as open as possible to maximize reuse. }\end{array}$ \\
\hline Goal: Good data management and stewardship & $\begin{array}{l}\text { Philosophy: OS, at least in part, views scientific output as a } \\
\text { commodity while IKDG research is primarily for community } \\
\text { benefit. }\end{array}$ \\
\hline Goal: Increased collaboration and consultation & $\begin{array}{l}\text { Epistemology: Scientific method (accuracy, evidence) versus } \\
\text { relational } \\
\text { Ontology: Realism and objective truth versus various } \\
\text { cultural subjectivities }\end{array}$ \\
\hline $\begin{array}{l}\text { Standards: FAIR-related: Reusability and } \\
\text { accessibility (standards) }\end{array}$ & $\begin{array}{l}\text { Materialities and Infrastructures: Intellectual Protection laws } \\
\text { \& licensing, funding, research design are misaligned. }\end{array}$ \\
\hline Orientation: Both processes are people and data & $\begin{array}{l}\text { Orientation: OS has a larger emphasis on standardized } \\
\text { protocols for data management: while people-centered } \\
\text { benefits are emphasized, the primary focus is the data itself. }\end{array}$ \\
\hline
\end{tabular}




\subsection{Vision}

The vision for open science, shares two key components: The first is a transformation of the scientific process, which is achieved by ensuring that scientific research, publications, data, and knowledge are publicly accessible with as few restrictions as possible. This includes a transformation of "scientific culture, methodologies, institutions and infrastructures" (CODATA, n.d.) in which science is planned, performed, and promulgated. The second key component is a societal transformation because of this openness. The open government action plan, which provided the impetus toward the adoption open science, is reflective of this orientation towards openness for social benefit. The action plan regards openness as a pathway to increased transparency, accountability to citizens which enhances citizen participation in policymaking and service design, and a more efficient and responsive government. These visions are carried into the primary goals and objectives of open science.

Meanwhile, the vision of Indigenous knowledge and data governance, generally, is for an environment in which the inherent right to self-determination and data sovereignty are realized and respected. The restoration of Indigenous governance over knowledge, data, information, and research pertaining to Indigenous peoples is the inspiration and motivation. The visions themselves are not necessarily misaligned, but they are likewise not aligned. However, the vision articulated in the Roadmap for Open Science is interesting as it states that the vision is to "maximize benefits for the well-being, health, and economy of our country" (Government of Canada, 2020, "Vision" para. 1). The vision neither aligns nor misaligns with the collective vision of knowledge governance, though it does generalize the best interests of the collective country and does not necessarily consider the diverse interests and priorities of Indigenous peoples on a nation-to-nation basis. 


\subsection{Goals}

Goals and objectives can be otherwise understood as the object of ambition and effort: the goals are the "end toward which effort is directed" (Merriam-Webster, n.d.). The goals of open science and Indigenous knowledge and data governance act as milestones towards achieving their respective visions and there are 5 shared goals between OS and IKDG.

1. To begin, both processes aim to maximize the benefit of data and reduce harm. It is articulated in all the OS documents analyzed that OS should increase reproducibility, reduce duplication, and maximize the benefit of scientific research output (OECD, 2015; Wilkinson et al., 2016; Government of Canada, 2018, 2020). The literature from the IKDG content analysis consistently demonstrates the same goal: to maximize research benefits by co-developing research strategies and engaging with Indigenous partners throughout the research process (NAHO, 2011; ITK, 2018; FNIGC, 2014) and to minimize harm by adhering to culturally developed ethical guides and protocols to protect privacy, confidentiality, and traditional knowledge (NAHO, 2011; ITK, 2018).

2. OS aims to collect quality, accurate, and relevant data in research, which is also indicated in the IKDG literature as a priority for achieving governance and selfdetermination in research.

3. both processes are meant to facilitate good data management and stewardship.

4. both processes aim to achieve increased collaboration and consultation to recognize a diverse array of perspectives.

5. Finally, open science and IKDG both seek to increase citizen participation and engagement in research-level processes.

While there is not complete overlap in the goals of OS and IKDG - for instance, greater scrutiny of research results is a goal of OS that is not explicitly stated in IKDG - it is significant to note that there is considerable overlap in the overall goals for both. 


\subsection{Purpose}

The purpose of OS and IKDG are completely different. The purpose of OS is to increase the accessibility of scientific research output and data to maximize their reuse and contribute to the overall public good. As federally funded science is paid for by the public purse, OS is a process which aims to make the results of this research accessible and usable for the people who pay for it. In addition, OS is commonly positioned as a remedy to issues with $21^{\text {st }}$ century science, as previously described. This includes issues with credibility, inaccessibility, transparency, and redundancy. Thus, the purpose of OS is to make science more credible, accessible, reusable, and transparent.

Conversely, the purpose of Indigenous knowledge and data governance appears to be the restoration of Indigenous control over knowledge and data that pertains to them. However, to see IKDG in this simplified way is somewhat reductionist. Beyond the restoration of control of knowledge and data, the purpose of governance is to divest from historical colonial institutional control, exercise governance of research to collect relevant and useful data towards the enhanced wellbeing of communities, and Nation Rebuilding. The purpose of Indigenous knowledge and data governance may differ from culture to culture or from one community to the next. For the sake of comparison, however, I broadly consider the purpose of 'Indigenous knowledge and data governance' to be threefold as described above,

1. for restoration of the inherent right of governance;

2. to divest colonial control; and

3. exercise governance over all aspects of Indigenous knowledge, data, and research towards enhanced wellbeing and Nation rebuilding.

In this regard, the purpose of OS and IKDG diverge, but that does not mean that IKDG is also not about accuracy or empiricism. Instead, the process and goal of knowledge and data 
governance is to ensure that Indigenous Peoples can exert control over the kinds of relevant and quality data that are being collected, to avoid the collection of irrelevant data, inaccurate portrayal of Indigenous populations (as deficit-based), and ensure that necessary data are collected about relevant phenomena in communities.

\subsection{Orientation}

While OS and IKDG differ with respect to their primary users - OS is meant to benefit the public while IKDG seeks to benefit Indigenous peoples, generally speaking - they are both primarily people-centered processes. While OS has standards for implementation that focus on the data management aspect of the practice, the concept of OS is to contribute to the public good. Similarly, IKDG is a people-centered initiative that aims to restore control of Indigenous knowledge and data to their rightful owners, towards the benefit and well-being of Indigenous peoples and communities.

\subsection{Philosophical Investigation}

The philosophical differences between Western and Indigenous science are important to understand as they directly relate to OS and Indigenous knowledge and data governance, respectively. Despite sharing a similar philosophical orientation, the Western worldview and Indigenous worldview of science are different. Linda Smith, an Indigenous woman, and scholar whose work is focused on decolonizing Western research methodologies to make room for Indigenous research perspectives and methods, demonstrates that Indigenous peoples "have a different epistemological tradition that frames the way we see the world, the way we organize ourselves in it, the questions we ask, and the solutions we seek" (Smith, 2000, p. 230). Indigenous epistemology is more holistic and relational, as "Indigenous peoples view the world we live in as an integrated whole" (Magga, 2009, p. 2). Conversely, Western worldviews are 
more focused on scientific origins and compartmentalized knowledge (Little Bear, 2009). Western science is often understood as the "attempt to search out, describe, and explain in natural terms generalizable patterns of events in the world" (Good, Shymansky, \& Yore, 1999) using empirically-based evidence and objective methods. It is also rooted in rationalism and seeks to uncover the objective reality that exists independently of what the observers do or desire, even if it cannot be fully known" (Maturana, 1990, p. 12).

In other words, Indigenous epistemology is more relational whereas Western scientific epistemology is grounded in objectivity, favoring methods that favor standardized accuracy and evidence-based conclusions. This is not to suggest that Indigenous approaches favor inaccuracy and do not require evidence; however, Indigenous approaches are more holistic, founded on ideals of interconnectedness, and does not necessarily strive for a universal set of explanations. Rather, conclusions are often more contextual and pluralistic.

Ontology, or the study of being, is concerned with what exists in the world and how humans can acquire knowledge (Moon \& Blackman, 2017). The ontological orientation of science is realism; Western science generally presumes that reality - the single, objective reality can be understood using appropriate methods (Nicholas, 2018). Conversely, Indigenous ontologies are difficult to fully describe or encapsulate, but the literature suggests that "Indigenous knowledge and the work of Indigenous thinkers (scholars, elders, community leaders, activists, community members) contain a wealth of place-specific practices for understanding how categories of being are made possible within diverse Indigenous cultures" (Hunt, 2013, p. 27). Indigenous ontologies share a person-land-ancestral inter-relationship that is holistic: no one element can be separated from the other. Indigenous ontologies integrate a "spiritual orientation that human beings have an important role in perpetuation of nature 
processes in the world" (Hart, 2010, p. 7). The biggest difference between the two, as observed within these specific research parameters, is that Western scientific ontology presumes that there is a single objective reality that can be understood with a standardized method, whereas Indigenous ontology posits that there are various ways of understanding and interpreting phenomena in the world, and that the way people see the word influences our individual subjective understandings of what can possibly exist (Hart, 2010).

Understanding the epistemological and ontological differences between the two is important because they influence the ways in which Western and Indigenous knowledge, research, and worldviews interact and conflict. As Hickey (2020) argues, "misunderstandings on an epistemological level are revealed as barriers. Learning more about the value of Indigenous epistemologies will help bring about the understanding required to address the misapprehensions between Indigenous People and Canadians of settler ancestry and create a respectful space for co-developed solutions to emerge" (p. 14). In other words, it is only through this recognition that meaningful co-development can occur in ways that prioritize the interests and priorities of both.

In sum, the philosophical orientation underscoring Indigenous praxis differs with the philosophical orientation of Western scientific praxis, including open science. This does not necessarily mean they are incommensurable, especially because applying critical race theory and tribal critical race theory allows us to identify the impacts of Western knowledge systems and policies and theorize new ways to decolonize these knowledge systems and policies to better serve Indigenous Peoples. Thus, while OS and IKDG have different philosophical orientations, understanding these orientations through the lens of postcolonial and critical race theories provides new avenues through which to theorize ways they can be applied together to create positive change for Indigenous Peoples. 


\subsection{Standards}

The standards facilitating OS and IKDG have different goals and processes. In this section I consider which of these standards and principles are interoperable and determine where OS and IKDG are incommensurable. As illustrated below, my reading and analysis of the literature suggests that open by default is incommensurable with OCAP, and CARE. This is because open by default necessarily circumvents the decision-making processes related to OCAP and CARE by requiring that data and research are made open by default where restrictions can be added later, if necessary. Although, open by default is tempered, as data that may reveal the privacy of individuals or about national security are not shared by default. The OCAP and CARE principles, which foreground the importance of Indigenous knowledge and data governance and allow for Indigenous individuals, communities, and organizations who own the data and knowledge to govern, control, and share them according to their own needs, appear to be incommensurable with open by default.

Table 4. Interoperability of OS and IKDG standards

\begin{tabular}{|l|c|c|c|c|}
\hline & OCAP & CARE & FAIR & OBD \\
\hline OCAP & N/A & Y & Y & N \\
\hline CARE & $\mathrm{Y}$ & N/A & Y & N \\
\hline FAIR & $\mathrm{Y}$ & $\mathrm{Y}$ & $\mathrm{N} / \mathrm{A}$ & $\mathrm{Y}$ \\
\hline OBD & $\mathrm{N}$ & $\mathrm{N}$ & $\mathrm{Y}$ & N/A \\
\hline
\end{tabular}

The above table illustrates the interoperability of OS and IKDG standards. For instance, OCAP is commensurable with CARE and FAIR principles because there are no oppositional or conflicting ideals amongst them. Ownership, control, access, and possession are not contrary to any element 
within CARE (collective benefit, authority to control, responsibility, and ethics) or FAIR (findable, accessible, interoperable, reusable). However, OCAP does clash with the Open by Default policy, as ObD does not explicitly allow First Nations to assert ownership, control, access, and possession of their knowledge and data in research. As such, the only notable misalignment among the standards is where ObD does not align with OCAP or CARE Principles. 


\section{Chapter 6: Discussion}

By comparatively examining the Government of Canada's Open Science and the objectives of Indigenous knowledge and data governance, I discovered that, while they differ, they are also compatible. Using a mixed methods approach I identified how the major vision, goals, orientation, philosophical foundations, and standards of OS and IKDG as articulated in the literature I selected for this analysis, and this is where I found that OS and IKDG are similar and where they are different. In this chapter I discuss these commonalities and differences and interpret, contextualize, and describe the significance of my observations and analysis in relation to the broader OS/IKDG discourse.

In the first section of this chapter, I return to the initial research questions and systematically compare the key components of both processes and discuss why and how OS and IKDG align and misalign. In the second section I will situate this analysis within some of the themes from the literature review including Western production of knowledge and decolonizing Indigenous knowledge, and The state of co-development methods in Canadian research. Here I discuss their relevance in a broader context and situate each practice in their different histories, 
motivations, theoretical frameworks, and target participants, and show how OS and IKDG are both people-centered processes which are, at their core, meant to contribute to the common good. However, the OS documents by the Government of Canada often lack fundamental consideration for Indigenous knowledge and data governance and clear strategies for facilitating effective Indigenous governance in the OS domain. In addition, the underlying institutions, and decisionmaking processes within the OS advocates are not inherently inclusive of Indigenous governance rights, and therefore may not meaningfully align with Indigenous interests in the long-term unless the expectations and guidelines for OS are co-developed from the start, or IKDG is implemented by design in OS frameworks as is open by default.

\subsection{Back to the Research Questions}

Through a content analysis and a semi-structured interview, I sought to answer two primary research questions:

Q1: How do the ideals of data, knowledge, and information compare with those of open science?

Q2: Is Indigenous open science possible?

The comparative content analysis aimed to answer the former question, and my reading suggest that:

Answer for Question 1: The two processes are not fundamentally opposed. Open science and Indigenous knowledge and data governance can be practiced together IF they are co-developed and standards, protocols, and practices that affect Indigenous peoples and their knowledge and data are prioritized. The decision-making capacity MUST be in the hands of the communities, individuals, and/or nations who are providing the knowledge, data, and information in the context of natural and/or social scientific research.

To better answer the second question, I chose to conduct a semi-structured interview to better understand whether Indigenous OS is possible. In this case I sought to situate my analysis 
within the real-life impact of data governance for Indigenous communities to understand how Indigenous governance functions, benefits, and relates to the wider 'open' movement. I chose this route because, while the ideals of open science and Indigenous data governance can be ascertained by analyzing literature, to better respond to the second question I chose to gain firsthand knowledge and explanation about the impact of data and knowledge governance from one of the founders of First Nations data governance, Elder Gwen Philips. Elder Gwen, as a pioneer of Indigenous data governance, is experienced in the field of data and understands her community's needs as they pertain to data governance and open data in Canada. Therefore, to investigate the impact of data - and government-mandated open data - on First Nations Peoples, is the first step in understanding the needs of First Nations Peoples to practice an Indigenous open science. The response to Question 2 is as follows, and how I came to this response is explained in the section that follows.

Answer for Question 2: Indigenous 'open science' is possible if the framework and standards are co-developed from inception. This means that the needs, priorities, and principles of Indigenous governance are foregrounded in the development of open science standards, principles, and outcomes to ensure that open science and Indigenous governance can be mutually beneficial, and that Indigenous participants in OS research have the power to exercise governance over the entire research process including contributing to the research agenda to ensure necessary data are collected, and exercising control and authority over how the data are managed, stored, and disseminated. It would be beneficial, and in keeping with the vision of IKDG, for data collected about Indigenous Peoples prior to OS to be closed unless the participants and/or collaborators in research decide (or have already decided) otherwise.

\subsection{Semi-Structured Interview: The Impact of Data Governance}

According to Advisor Gwen Phillips of the Ktunaxa Nation, any governance discussion should be underscored by the knowledge that governance is fundamentally about identity. Phillips wears many hats, but among her many ongoing roles she is the Provincial Champion of the BC First Nations Data Governance Initiative (BC FNDGI) and served as the Director of 
Governance Transition with the Ktunaxa Nation. The collective work of the BC FNDGI - lead by Gwen - is focused on fulfilling the vision of developing and implementing a "model of development and wellness that is self-governing, community driven, and nation based" because "the autonomy to define community wellness and the right of First Nations communities to own, control, access, and possess information about their peoples are fundamental to selfdetermination and to the preservation and development of their culture" (BC FNDGI, n.d., About). Through a series of stories about her work in the field of data governance, Gwen explained that "all [of] the symptoms we're suffering, as societies are because of [a] deep, deep concept of lack of recognition of who we are" (Phillips, 2021, personal communication) which can be remedied with the recognition of Indigenous data governance as an inherent right. Thus, governance plays a critical role in advancing positive outcomes, though the extent of the impact of governance for Indigenous communities is often reduced to what can be conveyed in academic literature alone. Gwen's stories about the impact of governance for First Nations communities provides the narrative and situated connection between the theoretical and practical impacts of governance that has been missing thus far from this thesis and describes the impact that governance can have on Indigenous communities. It should be noted here that I did not interview anyone from the OS profession, who may have provided a similar linkage between the theoretical and practical impacts of OS. However, as there is substantially less research on the impact of OS on Indigenous knowledge and data governance, I made the choice to address this gap with this thesis.

Gwen's work in the field of data governance began in the 1980's when she was the Director of Education for the Ktunaxa Nation. Before there was much scientific data on the topic of fetal alcohol syndrome, Gwen and the Ktunaxa Nation communities had recognized that there 
was something going on within the youth population that seemed to manifest in school and employment-related difficulties. Assumptions were made about it being related to alcohol consumption. It is now known that settler-colonial contact in North America is when alcohol was introduced to Indigenous Peoples and therefore drinking was a learned behavior (Frank et al., 2000). Historically, alcohol was also "jammed in the faces" of Indigenous Peoples; it was a trade good. The use of alcohol exponentially increased in correlation to colonization, especially during and following residential school and sixties scoop trauma, which further entangled alcohol and drug abuse with trauma. This meant that "alcohol and drug abuse and despair became predictable outcomes to systematic, multi-generational oppression" (Duran, 2006). However, at the time, the depth of alcoholism on reserve was not fully understood, and the correlation between alcohol use during pregnancy, and brain development had not yet been explored or understood fully. However, the Ktunaxa peoples have stories; Ktunaxa knowledge, that provide them with guidance on how to have a healthy pregnancy and how to raise healthy children. Consequently, the Nation sought to address the deeper social determinants of the circumstances they were noticing and began to look for someone to support the Nation's research to further understand the causation and the correlation and to demonstrate that it was, indeed, what was occurring.

A research study was officially undertaken by a husband-and-wife team of medical doctors and others, to uncover the effects of fetal alcohol exposure on the Ktunaxa Nation, which engaged the participation of approximately 170 school-aged community members. As Gwen recalls, the understanding was at the time, that this was a Ktunaxa process and that we would always be in control of the research and outcome. As the ones who promoted the research for the 
benefit of the community, and engaged the help of external researchers to support them in their

Nation-based research initiative, the expectation was that:

we're in control of this...our people are telling their stories and they will own their stories. And because we acknowledged that this initiative was done under what I call the Community Healing and Intervention Program, it was part of our healing journey. So, it was about us recognizing what colonial processes did to us, at not just a social level, but an organic level to understand what's now happening to us (Phillips, 2021, personal communication).

At the end of the research study, however:

The data was treated like research data and destroyed as per the schedule for records retention. It wasn't treated like personal health records even though that is really what they were. And we didn't know that at the time...I felt almost personally responsible in some ways. Because I love and know these people so well, because the people that I helped to fight for, for a different school system to fight for... for all of these things. They're my family. And so, it was like, this can never happen again - we need to control our data (Phillips, 2021, personal communication).

The Ktunaxa Nation wished to keep the data collected throughout the course of the research study on behalf of the people whose stories we were helping to tell. Data, especially in the field of social science, is invaluable to Indigenous communities because they often struggle with the capacity to conduct the kind of research that is desperately needed. For reasons including inaccessible funding, Euro-centric research agendas, and a lack of capacity in First Nations communities to conduct research themselves, foundational datasets are frequently missing from Indigenous communities. In this example, the recognition of First Nations governance over research and data would have afforded the community the chance to retain copies of the data for future use. This example also illustrates that, even if all Western ethical standards and protocols are followed, the outcomes may still be unethical according to the interests of Indigenous participants. This is because only individual communities can determine what is ethical, or in their best interests, or potentially harmful. When research is conducted with external researchers 
and subject to the Tri-Council Policy Statement: Ethical Conduct for Research Involving Humans (TCPS2), research data must be destroyed after the project's completion unless this is otherwise agreed upon. However, the destruction of the data - though ethical according to Western standards - was considered unethical by the community who provided the data in the first place as these were invaluable and collectively owned by the community, even though these ownership rights were not recognized in the eyes of Western standards. First Nations governance is therefore essential throughout the entire process: from the development of the research project to governance of the research data upon its completion.

The importance and impact of governance, therefore, cannot be overstated. As Elder Gwen demonstrates, data governance is crucial in its capacity to facilitate nation rebuilding and identity reclamation. A history of historical erasure, compounded with a history of detrimental research experience with Western researchers, has resulted in a deep lack of cultural identity as many Indigenous Peoples struggle to understand who they are. As described in Chapter 3 of this thesis, Indigenous languages are rapidly being lost, intellectual property has (and continues to be) exploited and extracted by non-Indigenous Peoples for personal and professional gain, and Indigenous data are often owned and controlled by non-Indigenous Peoples and often destroyed according to non-Indigenous standards of practice. Governance is therefore about the "assertion of ownership of your identity. And it is about...nation rebuilding by taking ownership of our intellectual property. And so, data sovereignty really is about assertion of...your supreme ownership of your identity. Nobody else gets to claim that or do anything with that describe you anymore" (Phillips, 2021, personal communication). In this way, data governance is about acknowledging that no one person, or external body owns the knowledge, culture, and languages 
of First Nation people: it cannot be taken away (again) when it is collectively owned by the Nations themselves.

Furthermore, governance of research is impactful because the research being done about, for and sometimes with is often misaligned with the needs and priorities of First Nations communities. The approaches to problem solving are different, so the research being done, and outcomes being realized, are not always in line with First Nations worldviews. For instance, Gwen describes the exasperation of research fatigue, because "they've been doing research on us and continue to do research on us...They've studied us to darn well, death" (Phillips, 2021, personal communication). But due to a fundamental difference in the methods and approaches to problem solving by Western and Indigenous Peoples, Western research often fails to yield tangible and meaningful benefits for the communities they seek to help. For instance, Gwen describes how

The provincial government notices a problem with unemployment, so they invest money in unemployment. They see a problem with homelessness, so they invest money in street programs (Phillips, 2021, personal communication).

The approach is to get rid of the problems that BC (most governments) has by viewing wellness through a Western lens, solving a problem with a monetary solution. But this approach is indicative of "not understanding the intricacies of the determinants of health and wellness" (ibid) for First Nations communities. In the First Nations' worldview of health and wellness, "it's not just health as [the] absence of disease. It's that intricate concept of what wholeness is, and wellness is" (ibid) as it is understood by our own communities. People of the Ktunaxa Nation, for instance, noticed an increased rate of unemployment or homelessness and recognized the importance of the underlying factors which contribute to these problems. By applying a culturally specific and contextual worldview, means that nations are the ones to define what 
strong healthy citizens are: there is a need to solve culturally specific problems using culturally specific solutions, which will result in more meaningful and holistic wellbeing for Ktunaxa (First Nations) Peoples.

This type of governance not seen in evidence informed OS literature. Governance is more than a politically motivated assertion of the right to control data and/or restricting others from using them. Data governance is more than simply taking back the right to control who can and cannot access local and traditional knowledge, data, and research. Governance, as demonstrated in this case study, is about what those data, and that knowledge, and research mean to communities who contribute to their collection. In the face of the historical erasure of Indigenous identity, it is about "asserting who we are instead of being told who we are" (Phillips, 2021) and self-determining using their own research, data, and knowledge. Considering persistent, culturally specific issues that are not adequately addressed by federal and provincial programming, governance is about controlling research initiatives to solve culturally specific issues with culturally specific and relevant solutions, supported by appropriate and accessible data. Finally, considering historical erasure and inaccurate representation of Indigenous identities, governance is about controlling the narrative of First Nations Peoples: more than anything, "it's really about us being able to tell our story, validate our story, and for others to not say, that didn't happen" (Phillips, 2021, personal communication). This is the impact of governance, and therefore Indigenous governance needs to be prioritized in open science, open government, and open data movements in Canada. 


\subsection{Similarities}

An important discovery from this reading is that Indigenous knowledge and data governance and open science are both people-oriented initiatives that aim to achieve collective social benefit, and the purpose for both is to increase the social well-being of the people the research is designed to benefit.

OS is a movement towards better quality science, increased collaboration among partners, and engagement between research and society. The OECD policy paper and Government of Canada's mandates for open science specifically indicate that OS should promote open citizen engagement with research output, maximize social benefit and return of scientific research outputs, and promote a broader understanding of science and enable citizen science (OECD, 2015; Government of Canada, 2018). The policies and standards supporting OS, such as the FAIR principles and open-by-default, are designed to facilitate this openness for social benefit and aim to make science accessible to the public. This is what Elinor Ostrom (1990) refers to as the knowledge commons, which is achieved when "intellectual and cultural resources are collectively managed, shared, used, and governed by all or most members of a community" (Albornoz et al., 2019). Open and collaborative science (OCS) has the potential to contribute to a more inclusive and diverse knowledge commons by facilitating more diverse forms of participation in, and circulation of, scientific knowledge "that [has] traditionally excluded actors from outside powerful and wealthy research institutions" (ibid). For this reason, it is argued that OS can be particularly beneficial for those who have been traditionally excluded from scientific knowledge production, as OS aims to facilitate citizen science and participatory research methods which can help communities address their local context-specific issues. In sum, the 
primary goal of OS is social benefit through enhanced research and data sharing and dissemination, while the secondary benefit of OS is economic in nature.

While the concepts of Indigenous knowledge and data governance aim to benefit Indigenous peoples rather than the public, the fundamental purpose and vision is to enhance Indigenous well-being. The core tenet of knowledge and data governance is that, through restoration of the inherent right to control their own knowledge and data (or knowledge and data about them), First Nations, Métis, and Inuit peoples can strive to reach self-determination and sovereignty in research which directly results in the overall health and well-being of Indigenous peoples and communities. The goal of research itself should be to enhance the overall wellbeing of the community and produce relevant and useful knowledge to achieve this goal. For instance, the NAHO's Principles of Ethical Métis Research sets the expectation that research should be relevant, accurate, responsible, protect Métis cultural knowledge, and benefit all (NAHO, 2011). The governance over research objectives enables First Nations communities to ask and answer their own questions to learn more about their communities (Phillips, 2016). Elder Gwen Phillips describes the importance of relevant research and data about First Nations communities and peoples, as a lack of this research and knowledge has led to "a sense of not belonging, a lack of connection... and a lack of recognition of who we are" (Phillips, personal communication, May 2021). Similarly, Inuit communities and organizations are increasingly expressing the need for enhanced social science and health-related research to address Inuit priorities of mental, emotional, and physical wellness. However, ITK's research strategy illustrates a biologicalphysical science research bias which has resulted in the majority of federally funded polar research being singularly focused on the physical and natural sciences, despite the clear need for social science research for communities (ITK, 2018). Enhanced Inuit governance over research 
would therefore directly impact the wellbeing of Inuit communities through the development of research projects that address Inuit priorities and interests.

Thus, while the standards, protocols, vision, and goals may conflict, it is worth noting that on a fundamental level, both OS and IKDG share a similar philosophy. I argue that this similarity suggests that both processes are commensurable, in some capacity, since they are striving for a similar social benefit related end goal. The potential for OS to enhance participation, collaboration, and inclusivity for social benefit aligns with the fundamental principle of Indigenous governance of data and research for community wellbeing and cultural rebuilding because they both agree that research should be for social benefit. This is significant because it suggests that OS may have the potential to better include Indigenous-specific priorities and interests than 'traditional' Western science (Chan, 2019) as OS is meant to be more inclusive, and in theory should open all kinds of science rather than just Western science. In this regard, OS and IKDG are theoretically aligned in their goal of producing relevant, timely, and accurate research for actionable social benefit.

\subsection{Misalignment}

While OS and IKDG align theoretically in the ways previously described, there are noteworthy areas of misalignment in practice. These include conflicts in the governance of knowledge and data, conflicts in the governance of research including research design and decision-making, and misaligned 'beneficiaries' for each process.

\subsection{Beneficiaries of OS and IKDG}

While the philosophical roots of both OS and IKDG is to produce and share knowledge and data for social benefit, both processes have different beneficiaries. In the case of OS, 
scientific output and knowledge is understood to benefit the greater good, by sharing knowledge for increased innovation, maximizing the accessibility of federally funded scientific output, and fostering more credible and transparent science. The 'audience' for OS, and the people who stand to benefit from this openness, is the public who are the benefactors of federally funded research, and other scientists who can access and build on other scientists' research, and research institutions. Conversely, IKDG as described by Elder Gwen Phillips, is solely for the benefit of First Nations, Métis, and Inuit peoples. Unlike OS, which aims to make scientific knowledge open to as many people as possible, IKDG aims to ensure that Indigenous peoples can easily own and access their own knowledge and data, including data and research about them, and can exercise their right to practice governance throughout the research process, including the choice to make some data public and some only to those concerned with a particular research project. Knowledge and data may be restricted from the public in ways that Indigenous communities see fit, as they are the inherent rights holders of this information. IKDG is premised on the fact that data sovereignty and governance directly result in positive outcomes for Indigenous peoples for two main reasons: 1) the right to govern their own knowledge makes accessing existing data and research easier, and 2) can help shed light on cultural concerns that have not been previously studied.

Additionally, the government documents articulate openness as a critical societal transformation resulting in increased transparency, accountability to citizens to enhance citizen participation in policymaking and service design, and a more efficient and responsive government. Though this concept and vision are not in opposition to IKDG, Elder Gwen Phillips reminds us that we should remain conscientious about who this transparency and accountability is for. It is assumed that by increasing transparency and openness in government processes, there 
should be greater accountability of the Government to the population it governs. Yet this does not stipulate a greater accountability between governments and Indigenous Nations, whose calls for accountability are not answered by current government commitments. For example, Elder Gwen spoke of her communiqué with an Indigenous services minister regarding data about water quality and health impacts. Without the data, she says, there is no way to prove one way or another what is happening to Indigenous communities who are drinking that water, so this government official was asked about the collection of these data. He replied that due to Indigenous data sovereignty, the department had no data on water quality and health impacts on reserve (Phillips, 2021, personal communication). This exemplifies an erroneous understanding of data sovereignty and jurisdictional responsibility.

In another example, she discussed a struggle with the government regarding ownership over residential school survivor testimony. Physical copies of the residential school survivor testimonies, which played a key role in the development of the Truth and Reconciliation Commission, are set to be destroyed per federal research ethics protocols. However, the importance of protecting these testimonies, in her eyes, cannot be overstated: as a critical piece of Canada's history, what could happen if the testimony - the proof - is destroyed? The ramifications of potential erasure and forgetting of this history are catastrophic which is why Elder Gwen has argued for years that "it's got to become part of our collection of our collective ownership over this data" (Phillips, 2021). This, she says, is government accountability and transparency to Indigenous peoples.

These examples illustrate the difference between the stated beneficial outcomes of openness in the Canadian government versus the desired outcomes being requested from Indigenous peoples to the government. As OS is positioned as a movement for the benefit of 
Canadians, it remains important to consider the ways in which it may - or may not - benefit Canadians equally. If this openness and accountability does not extend to the relevant needs and priorities of Indigenous communities, who desperately need access to data about/for them, who really stands to benefit? Until Indigenous interests are considered and accounted for in OS processes, and governance of knowledge and data is effectively exercised by Indigenous Peoples, it is reasonable to argue that OS and IKDG are not meaningfully aligned, at least until which time IKDG principles become a normalized part of OS.

\subsection{Governance of Knowledge and Data}

For OS and IKDG to be aligned, Indigenous knowledge and data must be governed by Indigenous Peoples. While neither the National Action Plan on Open Government, nor the Roadmap for Open Science, include a clearly articulated governance structure or process for federally funded OS research, it appears that OS research output will be held by the federal government, and federally funded researchers and their institutes and ought to be shared in an OS repository to make this research findable, accessible, interoperable, reusable, and open by default (Government of Canada, 2020). This is problematic since the FNIGC has specifically indicated that "The only way to prevent [governance] issues is to ensure data [are] not stewarded by the federal government" (FNIGC, 2014, p. 41). This is because "the Access to Information Act (ATIP), and the policies and procedures that support it, hinder the ability for First Nations individuals to exercise ownership, access, control and possession over their information and data. Furthermore, the Library and Archives Canada Act (LAC-A) presents an issue because it:

mandates that all records (including electronic) in the control of federal departments/institutions be transferred to the Archives when they are no longer used by the department/institution. Once transferred to the Archives, even personal information becomes vulnerable to an ATIP request, because the Privacy Act does not protect the privacy of personal information if the person 
has been dead for more than 20 years. This means that through ATIP and the LAC-A, even personally identifying information about First Nations people can be released ( 20 years after death). Since the federal government collects so much information about First Nations, this exposes First Nations and First Nation families to significantly greater potential harm and continuing problems with without oversight or limitation (30).

For this reason, the federal stewardship of Indigenous knowledge and data can hinder Indigenous peoples from exercising their inherent right to govern their own knowledge and data, either according to OCAP principles or community-specific protocols for governance. This is an area of misalignment between OS and IKDG, as IKDG cannot be exercised so long as OS research and results are stewarded by the federal government and federally funded researchers doing research about Indigenous peoples without them as part of the process.

It is important to acknowledge however, that there is immense potential for a data repository with cultural protocols controlled by Indigenous communities and individuals. A prominent example of this in action is the application of cultural protocols of the MUKURTU CMS for Indigenous communities to collect, preserve, and share their knowledge, data, and information in online databases that prioritizes the interests of those communities. Most significantly, MUKURTU includes protocols to indicate how certain kinds of information should be shared, and who it can be shared with. While the option exists to make cultural knowledge openly accessible, it is explained that "if items will be shared publicly, protocols for (open) public use and access need to be created and selected - it's never a default setting and is a decision that is always made" (Wynne, 2020, 9:40). This demonstrates that an open data repository can prioritize unique cultural needs of a variety of knowledge and data artefacts, and that can be built into the entire data process processes including consent and the interface. 
The conflict of data stewardship is a point of contention regarding the infrastructural capacity of some Indigenous communities. As mentioned, it is problematic for federal government to steward Indigenous data and it counters Indigenous knowledge and data governance issues since without possession of access and control are not always possible, and ownership needs to be negotiated for cultural information, knowledge, and data. However, First Nations, Métis, and Inuit communities do not always have the adequate capacity and infrastructure to effectively steward and manage their own data. This has been acknowledged by many (Engler et al., 2013; FNIGC, 2014; ITK, 2018; GIDA, 2019; Phillips, 2021) as a barrier to data governance in modern research projects and will likely remain an issue in the context of OS, until community data warehouses are developed, and Indigenous communities are supported in their data governance endeavors (Phillips, 2021).

To address this issue, data sharing agreements are encouraged in research projects where Indigenous communities wish to retain ownership over the data and research output. As articulated by the FNIGC (2014), through First Nation stewardship of First Nation data, First Nations can ensure that service contracts, data sharing agreements, licenses to use and other forms of legal agreement are in place whereby First Nations information is shared with other institutions and contain all the necessary provisions to protect First Nations OCAP ${ }^{\mathrm{TM}}$ of the data (p. 12). Thus, by developing a data sharing agreement in which First Nations, Métis, and Inuit communities can agree to third-party possession of the data while maintaining inherent ownership rights over the data, these communities can ensure that their interests are respected and that the appropriate protocols for data sharing and reuse are followed.

Therefore, it is possible that, without data sharing agreements or another mechanism which would allow Indigenous peoples to retain ownership of the knowledge and data used in 
federally funded research, OS and IKDG may be misaligned. For this reason, the importance of data sharing agreements in any research that involves Indigenous communities, knowledge, data, or information cannot be overstated.

\subsection{Governance of Research}

Another potential area of misalignment lies in the hands of the decision-makers of OS research and outputs. Specifically, there appear to be two critical decision-making opportunities within the OS process that conflict with Indigenous governance praxis: deciding what research will take place (and therefore methods to conduct the research) and deciding how the research will be made available (including to whom the research will be available). These are exacerbated by the funding and infrastructural structures in place for federally funded research.

The current decision-making process for federally funded research primarily involves funding bodies and non-Indigenous researchers (NAHO, 2011; FNIGC, 2014; ITK, 2018). This has been identified as problematic by First Nations, Métis, and Inuit communities and organizations, as this renders the research agenda set and controlled by non-Indigenous peoples, even when research takes place in Indigenous homelands or involves the use of Indigenous knowledge and data. Specifically, the FNIGC has stated that "the problems with use of First Nations information stems from who is in control - and thus what gets done, how it is doneand who knows about it" (FNIGC, 2014, p. 9). This is directly related to funding priorities as researchers are frequently "presenting completed research designs, often already funded, for community approval rather than collaborating from the start” (FNIGC, 2014, p. Between 5-9). Similarly, NAHO has suggested that "educating Research Ethical Boards about Métis context" (NAHO, 2011) should be addressed to better align the priorities of the primary funding bodies with the actual priorities of Métis peoples in research settings, as the two frequently do not 
overlap. ITK have also communicated their exasperation over the fact that "decisions about Inuit Nunangat research priorities and corresponding funding tend to be made unilaterally by governments and research institutions that are external to Inuit Nunangat" (ITK, 2018, p. 8). This is particularly difficult for Inuit communities since, as non-academic institutions, "Inuit representational organizations are ineligible for most Tri-Council research funding... which prevents Inuit from independently accessing research dollars that are in some cases intended to benefit our communities" (ITK, 2018, p. 25). This means that the bulk of research that is done about the Inuit homeland and Inuit communities is conducted without the input of Inuit peoples, communities, and organizations. In sum, the priorities of federally funded research are determined and/or refined by Canadian funding agencies and ethics review boards which are frequently misaligned with the needs and priorities of Indigenous peoples.

This is noteworthy because this funding structure is not likely to change as Canadian open science progresses. Funders play a significant role in the implementation of OS (OECD, 2015; Ball et al., 2018) and OS adoption in Canada applies to federally funded science and research. In other words, the issues with agenda setting, funding, and research requirements that cause tension with Indigenous people pre-open science are likely to remain barriers in an OS context. This is a specific instance in which the two processes are at odds until funding for codeveloped and Indigenous-led research projects increases and is more accessible to First Nations, Métis, and Inuit peoples.

Additionally, the decision-making process for data management and sharing appears to be exclusionary. While the governance process is not clearly articulated in the mandates from the Government of Canada, Recommendation \#4 in the Roadmap for Open Science stipulates that scientific research output, including science articles and publications, should be made open by 
default upon project completion (Government of Canada, 2020). Open by default means "publicly releasing government data and information that is of value to Canadians, with information being withheld only for necessary privacy, confidentiality and security reasons" (Government of Canada, 2016). As the FNIGC has identified various instances in which First Nations information and data have been made available by the Government of Canada, it is reasonable to postulate that Indigenous priorities may not be adequately protected under this definition. Open by design, therefore, is clearly counter to the premise of First Nations, Métis, and Inuit governance which recognizes that the inherent right to make decisions about their own data, knowledge, information is imperative for sovereignty. For this reason, without recognizing Indigenous knowledge and data governance by design in a similar manner, this OS framework does not consider the inherent right to self-determination and self-governance in research and cannot be fully aligned with the goals and interests of Indigenous peoples.

In the Roadmap, Recommendation \#6 recommends that a framework be developed to identify when information should be exempt from the ObD protocol (Government of Canada, 2020). In January 2021, while this thesis was in development, the Government of Canada published A Framework for Implementing Open-by-Default with Federal Government Science which was drafted with Indigenous input. Section 4.2.2 of this framework lists considerations for Indigenous knowledge and data sovereignty and their governance in the open context. In a nutshell, the document acknowledges that "there is a rationale for restricting access if the research output contains or is informed by Indigenous knowledge and data, or could likely harm Indigenous communities and peoples if released" (Government of Canada, 2021, 4.2.2.1). This is important because it demonstrates the consideration of Indigenous knowledge and data governance in the context of open government and OS, suggesting that federally funded science, 
when informed by Indigenous knowledge or data, should be exempt from the open-by-default requirement. However, more clear direction is required because the document does not indicate who decides whether the open publication of data and research "could likely harm Indigenous communities and peoples if released" (ibid) but rather instructs researchers to "consult with Indigenous Nations and/or governing bodies as necessary when considering the release of Indigenous data and information or scientific outputs that rely on such data and information" (Government of Canada, 2021, 4.2.2.1). It is clear that Indigenous communities and individuals are the only ones that are in a position to "determine whether something is in their best interest" and have the knowledge and authority to balance the potential benefits and harms associated with the collection and use of their information (FNIGC, 2014, p. 12) so this should remain a top priority in any kind of OS research if the processes are to be mutually beneficial and respectful.

The framework is also significant because it legally recognizes the inherent right of selfdetermination and governance, and states that First Nation, Métis, and Inuit expectations for ethical research by acknowledging OCAP, the National Inuit Strategy on Research, and the Principles of Ethical Métis Research. In sum, while there are underlying institutions and processes which may hinder the ability for Indigenous Peoples to exercise full and meaningful governance throughout the entire research process, it is possible that First Nations, Métis, and Inuit needs, and priorities can be incorporated in OS mandates and frameworks and to codevelop a more inclusive OS in Canada that includes IKDG.

\subsection{Broader Implications of Findings}

In this section I return to the themes discussed in the literature review to contextualize these findings in the broader theoretical context. I argue that the positioning of OS as techno- 
solutionist and techno-utopian orientation may ignore the possibility that OS may embody new forms of colonialism and reinforce existing colonial ideologies and methods, which are barriers in traditional science when it comes to IKDG. Furthermore, I suggest that OS may prioritize the production of Western knowledge if Indigenous knowledge and data governance is not better supported by the institutions and policies that guide its adoption. Finally, I argue that if IKDG is not foregrounded, and these issues are left unaddressed, OS may reinforce Western knowledge, ideologies, and values.

\subsection{Techno-Solutionism and Techno-Utopianism}

In chapter 4 I identified that the vision of OS is it's potential to transform the entire field of modern science, including "scientific culture, methodologies, institutions and infrastructures" (CODATA, n.d.) and the general anticipation of OS to result in a societal transformation. Here, openness is regarded as an avenue to increase citizen participation and engagement with scientific research, the dissemination of citizen science output, and higher social and economic impacts of public research (OECD, 2015).

I return to this point to argue that the organizational literature on OS, as well as the directives by the Government of Canada, are demonstrative of technological solutionism and technological utopistic ideals. Technological solutionism (TS) is a reductionist view of technology which holds the belief that every problem has a solution based in technology. This theory builds on the reductionist theory of technology by Thorstein Veblen - technological determinism - which posits that in society, technological advancements are the primary determinant of societal change, institutions, and social values (Veblen, 1908). TS extends to complex social issues such as politics, public health, education, and law enforcement, and suggests that these can be understood as neatly defined problems with solutions that can be 
optimized by inevitable technological advancements and data sharing (Morozov, 2013; Cramer, 2014). In this theory, technology is viewed as an autonomous force that positively changes society, but itself is largely unaffected by social elements (Adler, 2006). Langdon Winner (1977) argued that "changes in technology are the single most important basis of change in society" ( $p$. 65) as the technology in society influences the way in which a given society exists. Similarly, scholars of the utopian tradition believe that technological advancements are the means of achieving a perfect society: techno-utopianism is based on the premise that technology enables society to achieve social, economic, political, and cultural advancements for the betterment of that society (Segal, 1986).

Indeed, the OS literature examined in this thesis espouses the view of OS in a technologically deterministic and utopian fashion. The content of these documents suggests that OS will advance society by “accelerat[ing] progress towards solving society's most persistent problems" and will act as a "catalyst for change in the way we practice science, how we create opportunities for innovation, and in how we build new partnerships in industry" (Government of Canada, 2020, para. 7). The goals of OS, which include “open citizen engagement, enhanced democracy, increased collaboration and consultation, inclusiveness, transparency, [and] scientific innovation", are also demonstrative of a utopian perspective, as they are articulated as guaranteed to result in social benefits that can only be achieved through the operationalizing of an OS and open government approach. The utopian vision for OS envisions an environment of enhanced innovation, well-being, health, and citizen engagement, where progress toward problem-solving is amplified (Government of Canada, 2020).

Scholars such as Mary Francoli and Tracey P. Lauriault (2018), Sonal Zaveri (2019), Leslie Chan (2019), and Jeremy de Beer (2020) have warned us about the confluence of 
openness with social change, inclusivity, and democracy. Critics of technological determinism and utopianism have long argued that these views conceal unequal economic relations (Mosco, 2005; Carey, 1989); more recently in the specific context of OS, scholars have continued this line of thinking. Francoli and Lauriault (2018), as critical open government scholars and actors have suggested that "while there is an association between greater openness and democracy, this may be an overly deterministic assumption (Garsten and Lindh de Montoya 2008 in Francoli \& Lauriault, 2018, p. 3). Furthermore, they remind us that Canada has a problematic past in relation to openness, as the "Harper government under which [open data and open government] programs flourished, was renowned for silencing public servants, quashing science, impeding access to information, and shutting down data collection institutions" (Francoli \& Lauriault, 2018, p. 4). Sonal Zaveri, a scholar of gender and equity in politics, contends that "in the same way that the diffusion of ICTs and their inclusion in development projects [do] not guarantee equitable benefits...open processes take place in the larger context of systemic inequities and do not necessarily transcend them" (Zaveri, 2020, p. 109). Conflating openness with positive social change is dangerous because an overly deterministic and/or utopian view of OS may turn attention away from the inequities that OS may perpetuate and exacerbate. Openness itself is not a sustainable or effective mechanism to produce more diverse and inclusive OS and, furthermore, until the underlying inequalities of 'regular science' are addressed, they will extend to the open science movement and fail to effectively transform the scientific landscape (Zaveri, 2020; de Beer, 2020).

This is especially pertinent for Indigenous Peoples in Canada. In Chapter 3, I discussed Indigenous knowledge and data governance and the struggle in the context of mainstream codeveloped science, as many scholars have indicated a host of ethical and legal challenges with 
sharing co-developed research in online formats when that research includes Indigenous knowledge and data. These concerns include (but are not limited to) free informed and prior consent, privacy, and concerns over intellectual and cultural property (Browne, 2012; Engler, Scassa \& Taylor, 2013; Browne \& Ljubicic, 2019). Co-development in research, while beneficial, requires renewed consideration of best practices that meaningfully address these concerns as they relate to First Nations, Métis, and/or Inuit communities. For instance, inequities could include: the insufficient legal mechanisms with which to protect Indigenous knowledge and data; the lack of Indigenous-led research initiatives in science and health research; the power imbalance between researchers and Indigenous participants; and the infrastructural barriers which limit the ability of Indigenous Peoples to own and possess their own data and to exercise full and effective governance over those data and that knowledge. These kinds of inequities may be overlooked if OS is uncritically framed as inherently inclusive and equitable for everyone involved.

In sum, while it is argued that open science will result in a more inclusive science in which citizen and participatory science can flourish, it is important to acknowledge that there does not appear to be institutional change in the way that research agendas and funding requirements are designed. The positioning of OS as techno-solutionist or techno-utopian, by conflating openness with inclusivity and social benefit, could fail to acknowledge these existing inequities that disadvantage First Nations, Inuit, and Métis Nations. Unless a Canadian approach to OS is co-developed, the process will fail to be mutually beneficial for Indigenous Canadians alongside non-Indigenous Canadians. 


\subsection{Producing and Reinforcing Western Knowledge}

Also discussed in Chapter 3, is the common debate in the field of OS discourse is whether OS will diverge from the tendency of science in general to produce and reinforce Western forms of knowledge production, or to transform the scientific landscape by facilitating greater diversity and amplifying traditionally marginalized voices. Supporters of the OS movement tend toward arguing that OS will lead to more diverse scientific knowledge because it "provides a key opportunity to critically reflect on who is involved in knowledge-making processes, what tools are used, and what forms of knowledge are being produced and legitimized" (Hillyer et al., 2020, p. 357). Conversely, critical OS scholars contend that Western science, research, and academic institutions, even in the realm of OS, will remain sites of colonial dominance (Akena, 2012; Coburn et al., 2013; Kerr, 2014; Deb Roy, 2018; Mueller et al., 2019) although in Canada universities are undergoing a process of Indigenization. The latter argues that OS "continues to act as a tool of colonialism, particularly by privileging Western science, institutions, and administrative procedures" (Mueller et al., 2019, p. 399) which can be recognized in the way that "colonial ontologies, epistemologies and methodologies are reproduced as 'best practices' and where the priorities of colonial states and capitalist social relations, including the need to show that research is profit-making, motivate research and teaching" (Coburn et al., 2013, para. 14). The result is that most scientific knowledge is produced according to Western ideologies, values, and objectives, and that this centralizing of Western research in the scientific domain works to reinforce Western knowledge as dominant across disciplines (Akena, 2012; Deb Roy, 2018). Finally, they argue that "Indigenous research is measured against the colonial sciences, which are taken for granted as the research standard and then against this standard Indigenous research is found wanting" (Coburn et al., 2013). 
These tendencies are the embodiment of new colonialism, which results in the imposition of Western knowledge and policies on Indigenous populations despite their frequent inability to fulfill the needs of these Indigenous communities (Phillips, 2017).

This necessitates the question: is OS another form of new colonialism? While this thesis does not conclusively argue in favor of either camp, my findings suggest that the current orientation of OS, in the literature I examined, certainly has the potential to reinforce the hegemonic ideologies and values of Western knowledge producers. A deeper analysis of OS output will have to be conducted to better assess if this bears out in facts. Nonetheless, what I did observe is that the institutions and standards advocating for OS adoption in Canada are oriented towards Western researchers and Western forms of knowledge production. Especially since Indigenous governance and sovereignty is absent from OS mandates: the Roadmap for Open Science does not address Indigenous data governance or sovereignty although it is mentioned in another government directive for open government. This is concerning as departmental OS action plans will build on the direction included in the OS roadmap; and not necessarily the open government directives, therefore a failure to acknowledge OCAP, CARE Principles, or Métis and Inuit specific principles of ethical science research and governance in the OS plan may lead these principles being absent in departmental action plans. In this way, it is reasonable to postulate that OS may in fact represent a new colonialism in its potential to prioritize nonIndigenous values, needs, and priorities in the OS mandates, standards, and directives, especially if not explicitly stated.

Furthermore, the configuration of OS funding and direction means that federally funded scientific research agendas are more often reflective of non-Indigenous perspectives, even when research takes place in Indigenous homelands, or includes Indigenous peoples as consenting 
participants (FNIGC, 2014; ITK, 2018). Elder Gwen Phillips argues that, unless OS restores the decision-making power to Indigenous peoples and acknowledges the right to governance, it is colonial and antithetical to Indigenous knowledge and data governance. Thus, where meaningful acknowledgement of Indigenous governance would position Indigenous Peoples to exercise governance over research and assert their specific research needs, the OS research process appears to be unilaterally governed by Tri-council funders who will play a significant role in the implementation of OS (OECD, 2015; Ball et al., 2018). Therefore, it is reasonable to suggest that most research agendas will be determined by these funding agencies.

However, this does not mean that OS will predominantly facilitate the production of Western-centric knowledge. In 2017 the Canada Research Coordinating Committee (CRCC) was created to unite the presidents of the Tri-Council research granting agencies (CIHR, NSERC, and SSHRC) with other governing bodies to oversee the research granting process. A key priority of this committee in 2017 was to reaffirm that "federal granting agencies' commitment to the Calls to Action of the TRC with the creation of a national dialogue with Indigenous communities to co- develop an interdisciplinary Indigenous research and research training model that contributes to reconciliation” (Government of Canada, 2019, 'Starting the Journey'). This is significant because it led to a 2018 budgetary commitment to developing a strategic plan to identify "new ways of doing research by and with Indigenous communities" and to "grow the capacity of First Nations, Métis, and Inuit communities to lead their own research and partner with the broader research community" (ibid). As a part of this initiative, a Strategic Plan (2019-2022) was developed, and its priorities focused on building new relationships with Indigenous communities, developing strengthened models for co-development of research, and supporting Indigenous priorities in research. 
The Strategic Plan indicates an end date for 2022, meaning its outcomes should be achieved by this time. It is however currently unclear how well-developed the plan's priorities are. However, this remains an important document to acknowledge because it speaks directly to the issues that have been raised by First Nations, Métis, and Inuit peoples regarding the predominantly non-Indigenous research priorities in federally funded research. While most of the scientific research in Canada has been, until recently, primarily developed and decided by nonIndigenous researchers, it is possible that the adoption of OS can coincide with the enhanced development of inclusive funding opportunities so Indigenous priorities can be better included in co-developed scientific research in the coming years. This could make OS a truly more inclusive method of science that better includes the priorities and voices of non-Western researchers and participants, one that moves away from producing and reinforcing Western forms of knowledge.

In sum, it is possible that OS could function as a mode of new colonialism if knowledge and data governance are not factored into the development of OS standards and policies at the source. In other words, while OS and IKDG are not inherently misaligned, OS may perpetuate new colonialism in the way that its policies, procedures, and standards fail to adequately acknowledge and account for First Nations, Métis, and Inuit goals, ways of knowing, and priorities. Consequently, the best way to develop inclusive OS is to acknowledge that there is no such thing as a "one size fits all approach to open science... it is instead, a flexible concept that should be adapted to reflect local norms and realities" (Hillyer et al., 2020, p. 372). It is through this development of standards and processes that reflect local norms and realities that OS in Canada can be developed in a way that diverges from traditional science's tendency toward production, reinforcing, and legitimization of primarily Western knowledge, values, and priorities. 


\section{Chapter 7: Recommendations, Future Research, and Conclusion}

The modern resurgence of open science, led by advancements in digital infrastructure and technology, has gained momentum. It is lauded by scientists and researchers for its potential to remedy $21^{\text {st }}$ century issues with the scientific method: from increasing the transparency of scientific processes, making research outputs more accessible to as many people as possible, increasing the findability and reusability of federally funded research to maximize its benefit, removing barriers to amplify innovative potential, and making scientific research more credible by making it more open to scrutiny from the scientific community. OS is the path to a type of scientific utopia. It is certain that, as OS continues to eclipse the closed-and often irreproducible scientific practices of the 20th century, the future of science appears to be one that prioritizes openness.

It is important to scrutinize this technologically-utopian movement and rather than look to OS to solve the widely regarded 'problems' with closed and traditional science, and it is important that the underlying methods, processes, and infrastructures of OS and the environments in which they operate be critically investigated. In Canada, for instance, the 
enthusiastic adoption of OS coincides during a time of governmental reconciliation, whereby First Nations, Métis, and Inuit peoples are increasingly pushing for self-determination in research, and knowledge and data sovereignty and governance. Historically, First Nations, Métis, and Inuit peoples have been subject to colonial research methodologies, treated as research subjects rather than collaborative partners, have been subject to extractive research practices, and have often struggled to access data to which they contributed (Drawson et al., 2017; Hyett et al., 2019; FNIGC, 2020). It is therefore important to consider the ways in which the adoption of OS in Canada might influence the capacity for Indigenous peoples to exercise effective governance over their own knowledge and data in the context of OS research.

Examining this scientific movement through the lens of critical theory, especially the social construction of scientific knowledge, postcolonial theory, and critical race theory/tribal critical race theory, is important. First and foremost, by foregrounding the OS movement with the knowledge that scientific knowledge is influenced by the social environments and cultures in which it is produced, and that it is not simply objective and neutral facts about the natural environment, acknowledges that an OS movement may tend to bias Western scientific knowledge and reproduce the dominant ways of thinking. As the Western world is leading the OS movement, it is entirely possible that the OS policies and processes in place could serve to make Western science - not 'all' science - more widely accessible, reused, and reproduced across the globe. By first understanding that Western science is simply one form of science, and not more truthful or rational than Indigenous science, we can consider the ways in which the OS process may or may not equally facilitate the production and dissemination of other kinds of knowledge and science. 
Secondly and similarly, applying postcolonial theory helps to account for the political and social impact of colonial laws, policies, processes, and knowledge systems on Indigenous knowledge systems, laws, policies, and processes. The application of postcolonial theory on the concepts investigated in this thesis has allowed me to theorize the ways in which institutions, policies, and procedures for implementing OS may prioritize the needs, values, and interests of Western researchers, thereby perpetuating legacies of academic, institutional, and scientific colonialism. Unpacking the OS process considering the colonial history of Indigenous Peoples in Canada allowed me to better understand the ways that a postcolonial OS can function to better include the needs of Indigenous partners in research and data management, more broadly.

In addition, critical race theory and tribal critical race theory provide a mechanism by which Indigenous Peoples can act on these findings. Considering the process of OS through these lenses illuminates important considerations about the ways in which OS may or may not serve to benefit Indigenous Peoples and contributors to research as it may benefit Western scholars and benefactors of OS; these theories "offer the possibility of unmasking, exposing, and confronting continued colonization within educational contexts and societal structures, thus transforming those contexts and structures for Indigenous Peoples" (Haynes-Writer, 2008, p. 1). CRT and TCRT allowed me to identify the ways in which OS has the potential to accommodate and include non-Western needs, values, and forms of knowledge, creating a critical space in which Indigenous Peoples can utilize the OS movement to counter deficit-based representations and act as arbiters of their own knowledge and data, and the ways that these are portrayed, shared, and protected.

The final linkage between these theories is two-eyed seeing, which also creates the conditions which to recognize the strengths of Western methods and knowledge systems 
alongside the strengths of Indigenous methods and knowledge systems. Viewing these

knowledge systems together in the context of OS and IKDG reinforces the notion that they can in fact be practiced together: that OS is not inherently oppositional or contrary to the values and goals of IKDG. While they may not always fully align, applying the two-eyed seeing framework encourages us to look for the ways in which OS and IKDG can be practiced together to be mutually beneficial, and respectful of the ways in which they may not align. Viewing the strengths of each system, I believe, is the best way to co-develop an Indigenous OS praxis in the Canadian context.

\subsection{Research Summary}

This thesis has aimed to bridge the gap in understandings of OS and IKDG to foreground their similarities and differences. This research is timely and relevant as the adoption of OS in Canada is in its developmental stages, and there is time to reinforce IKDG now instead of backtracking. Furthermore, there is much discussion about the implications of open data and open government for knowledge governance, but there are currently no directives or standards (UNESCO is delivering a framework for Indigenous governance in the open science space) which are guiding the process, although FNIGC is working with the Open Government Team at the Federal Government on open data. With the growth of Indigenous self-determination and data sovereignty, makes this the perfect time to examine these questions and processes. In this thesis, I therefore sought to answer two primary research questions:

Q1: How do the ideals of Indigenous data, knowledge, and information compare with those of open science?

Q2: Is Indigenous open science possible? 
To answer these questions, I adopted a mixed methods approach where I conducted a content analysis and a case study. I chose mixed methods to perform a more robust analysis of both OS and IKDG; the content analysis allowed me to better understand the processes as articulated in literature, the case study provided a more nuanced and conceptual understanding of the impact and importance of Indigenous knowledge and data governance. For the content analysis I open coded 10 key documents - including organizational, grey, and academic literature - and from each extracted their vision, goals, purpose, orientation, philosophical traditions, and standards about OS and IKDG. I then comparatively analyzed these observations to formulate some conclusions about how OS and IKDG align and diverge. I developed a case study with the qualitative data I collected during a semi-structured, one-on-one interview with Elder Gwen Phillips of the BCFNDGI.

To answer my first question, I discovered that their goals, objectives, purposes, and visions of OS and IKDG do not effortlessly translate; at their core they are deployed for different purposes, different audiences, and are implemented with different mechanisms. However, this does not mean they are fundamentally adversarial. Just as traditional knowledge and Western knowledge can be bridged in research, and Indigenous and Western values can be articulated together, the priorities of IKDG can be braided within the OS paradigm. However, as stressed by Elder Gwen of the BC FNDGI, OS can only be aligned with Indigenous knowledge and data governance if First Nations, Métis, and Inuit peoples are able to exercise full control and jurisdiction over their knowledge, data, and information during the entire research process, which includes the results. It is through this exercise of governance that First Nations, Métis, and Inuit peoples can reclaim their cultural identities and self-identify according to who they are during research and in the production of knowledge about themselves. 
Regarding the second question, I discovered that frameworks and concepts posed by the BC FNDGI with Gwen Phillips, and the MUKURTU CMS - developed and maintained in partnership with the Center for Digital Scholarship and Curation at Washington State University with the Warumungu tribe in Australia - demonstrate very clearly that, yes, something called 'Indigenous open science' can and does exist. Indigenous 'open science' is possible if it is codeveloped from inception, which means that the needs, priorities, and principles of Indigenous governance are foregrounded in the development of open science standards, principles, and outcomes to ensure that open science and Indigenous governance can be mutually beneficial. Open science is also tempered in the simplest way as not all research data are disseminated openly, as there are privacy concerns, concerns about revealing the sites of species at risk, and the release of data that may have national security implications are also not published. Indigenous OS would temper open by default, in the same way, in terms of the publishing of data, but also in terms of ownership, access, control and possession in addition to Indigenous engagement in the full research process.

Also, while the two practices share some common goals, some philosophical orientations, and purposes, the only way for OS to align with and be respectful of IKDG is if the process of the governance over research is restored to the Indigenous peoples whose knowledge is being applied to or collected about. This governance extends to all aspects of research involving, or associated with, Indigenous peoples, communities, data, knowledge, and information. I deduced that 'knowledge governance' refers to several processes, rights, and expectations, in this case, that empower Indigenous peoples to assert control over the research decisions that impact them and their communities. This includes making decisions about what is best for their communities, collaboratively setting the research agenda to ensure that research is accurate, relevant, and 
reusable, and asserting jurisdiction over the knowledge, data, and information that inherently belongs to them.

This research is relevant because, as OS and IKDG proliferate in mainstream data management discussions, there remains some ambiguity about the ways in which these interact. On the surface, it may appear that the processes of OS and IKDG are divergent: as one is meant to make everything open, the other seemingly encourages more restriction, however, that is a superficial assessment. The general purpose of IKDG is NOT to hide all First Nations, Métis, and Inuit knowledge, information, and data from the world, but is instead to be sovereign over that knowledge, information, and data, and to make community specific decisions on how that knowledge, information, and those data ought to be managed and disseminated and for what purposes. The purpose of Indigenous governance is to restore the control over knowledge and data to their rightful owners, which was lost during the colonial years where Indigenous peoples were stripped of their cultures, ways of knowing and doing, and subject to colonial methodologies, ways of knowing, and research. Similarly, the process allows Indigenous Peoples to self-determine what kind of research is done to contribute to the overall understanding of their culture and communities, towards their goals of enhanced wellbeing and Nation and community rebuilding. As an exercise in reclaiming inherent rights and restoring power to Indigenous peoples in the postcolonial and reconciliation era, Indigenous knowledge and data governance is about setting the parameters for acceptable use: parameters which can only be determined by those who hold the knowledge and produce the data.

Thus, there is no better time to consider ways in which OS standards can be mutually beneficial for Western and Indigenous interests, and to further investigate the ways in which processes are fundamentally aligned and misaligned. 


\subsection{Recommendations}

Based on the findings and discussion presented in Chapters 5 and 6, the following is a set of recommendations for OS adoption and policy development in Canada:

\section{Recommendation 1 - Indigenous knowledge and data governance should be included, by design, in Canadian open science}

As OS is intended to be a more inclusive way of doing and sharing scientific research, Canada's open science mandates and directives should include Indigenous knowledge and data governance by design. The Framework for Implementing Open-by-Default with Federal Government Science is a great start, especially because it was written in collaboration with representatives from the FNIGC, however I suggest that in order to ensure that Indigenous Peoples' inherent right to selfdetermination (and by extension, knowledge and data governance in research) is recognized and actively practiced in the research process, Indigenous knowledge and data governance by design should be incorporated in the same way that open by design is inherently incorporated. Consequently, departmental Open Science Action Plans by the Government of Canada ought to include, at minimum, the acknowledgment and application of the CARE Principles for Indigenous Data Governance alongside the FAIR Principles. Federal scientists and researchers should aim to implement best practices and Indigenous interests in their work, and should be guided by the CARE Principles, unless there are existing community-specific protocols and ethical guidelines in place (which should take precedent). The OCAP Principles, Ethical Principles of Métis Research, and priorities described in the ITK National Inuit Strategy on Research (at minimum) ought to be incorporated when research involves any aspect of First Nations, Métis, or Inuit data and knowledge, respectively. Additionally, there should be clearer 
explanation about the governance of OS in Canada. Governance ought to clearly articulate and describe in federal directives to make it more clear to First Nations, Métis, and Inuit peoples in

Canada how the OS model may conflict with their own forms of governance. The description of the governance process of OS is ambiguous and scholars have acknowledged that "Little time is spent on assessing how open data itself is governed, or how it embraces open governance" (Brandusescu et al., 2019). Concurrently, the environment of governance, including licensing, ownership, and intellectual property protection, is ambiguous. This may present barriers to those unclear about how their knowledge and data will be governed and shared in the OS science domain. Until this process is made clear the implications (if any) that exist for Indigenous peoples striving for self-determination and effective governance over their knowledge and data will remain ambiguous.

\section{Recommendation 2 - Increased focus on infrastructural barriers to Indigenous knowledge and data governance}

There needs to be a focus on the instrumental and infrastructural barriers to Indigenous knowledge and data governance over research, knowledge, and data. This recommendation is related to two specific findings. The first is that there are underlying infrastructural barriers that continue to present obstacles for the effective knowledge and data governance by Indigenous peoples. Second, it has been demonstrated that the underlying processes by which OS is managed fundamentally conflict with the nature of Indigenous knowledge and data: for instance, IP and copyright laws do not extend to most forms of Indigenous knowledge. These issues should be further acknowledged in the OS context, and resolution to these issues should be grounded in Indigenous worldviews. 


\section{Recommendation 3 - Develop a strategy, database, or standard to support Indigenous knowledge and data governance in scientific research with metadata tags for Indigenous knowledge and data}

In relation to the previous recommendations, the Government of Canada ought to examine new avenues for research data management that meet the needs of Indigenous communities and participants, including, but not limited to, the right to data governance. For example, the MUKURTU content management system is a repository which incorporates cultural protocols to enable and empower Indigenous peoples to participate in data sharing on their own terms. While CARE principles and OCAP principles are meaningful ways to facilitate Indigenous knowledge and data governance in the OS domain, these principles ought to be understood as a starting point to further co-develop processes, standards, and infrastructures to fully and meaningfully engage and support the right of Indigenous peoples to exert control and governance over their traditional knowledge and data as it pertains to OS and open government, more broadly. Where confusion exists regarding the parameters of 'Indigenous knowledge and data', strategies and frameworks should continue to be co-developed with Indigenous organizations and the communities involved. To ensure Indigenous perspectives and voices are included in federally funded science, metadata requirements should include a form of tagging system whereby the use of Indigenous knowledge and data, and/or Indigenous partners in research, can be clearly indicated. This could serve as a concrete indicator of the inclusivity of open science research in Canada to facilitate the greater diversity of views and knowledge systems, while also providing a mechanism by which the application of Indigenous knowledge and data can be recorded and made closed by default, and opened when and if the Indigenous collaborators so choose. 


\subsection{Future Research}

The critical narrative on global OS is broad and nuanced. The process of OS itself is still in development, with various governance and policy-making bodies developing adequate and universal strategies for OS implementation (UNESCO, n.d.). As the process becomes more widely adopted and implemented, new concerns, critiques, and areas of further investigation will emerge. Consequently, this thesis is applicable to the specific context of Canada and the implications of OS on Indigenous knowledge and data governance here. It has focused on the unique tensions that may arise in the continued practice of data, information, and knowledge governance by First Nations, Métis, and Inuit populations in the OS and domain. Consequently, there are various avenues for future research in this area of study.

Future research related to the Canadian Indigenous context could also focus more specifically on the unique priorities and needs of individual First Nations, Métis and/or Inuit communities and Nations. As stated, the purpose of this research and approach was to address a knowledge gap, as there is scant evidence of the relationship between OS and IKDG in the Canadian context. For the sake of including the interests and priorities of all three of Canada's Indigenous groups, I chose to only analyze one specific organizational document from each of the representative Indigenous organizations. Future research would be more nuanced, and I would examine concepts of OS and IKDG with a focus on the situated histories, priorities, and needs of specific communities to have a broader understanding of OS, its impact and how it is mobilized in practice. This might include an analysis of local and traditional protocols for knowledge and data collection and sharing, as there may be additional protocols and principles for ethical research that have not been included in this research, most notably as the study here was document focused, more case studies and direct communication with data and research 
governors in community would better lend itself to more understanding. The interview with Elder Gwen Philips illustrated the benefits of situated, experiential and professional knowledge about IKDG.

While the focus here was on the congruence of OS and the praxis of Indigenous knowledge and data governance, and the implications and outcome of this research could be beneficial for other communities in Canada that may be 'marginalized' and not prioritized in the development of OS in Canada, and who may rightly so, may want more autonomy over the research process as seen in the Arctic research conducted by the GCRC. Future critical OS research could be useful to unpack the broader implications of OS on other marginalized communities by focusing on other specific cultural groups who may have their own culturally specific requirements and concerns in the context of research and OS. Specifically, there is a rich body of work in the field of scientific racism, critical race theory, intersectional disaggregated data, data feminism, which could be explored in the context of OS, as structural racism in scientific institutions in Canada is documented: Chakravartty et al. (2018), Roberts et al. (2020), Etoroma (2020), and others have demonstrated that systemic inequality pervades social science research fields of communications, psychology, and education, respectively. In the vein of critical OS discourse, future research could address and unpack the potential for OS to remedy or exacerbate these instances of structural and institutional racism as it pertains to Black, Asian, and other racialized communities in Canadian science research to contribute to a more robust understanding of open science's potential for inclusion and exclusion. OS, equity and inclusion might be another avenue.

Finally, I acknowledge that there are a multitude of different obstacles and concerns internationally relating to OS adoption. Scholars have criticized the potential for OS to 
"contribute to scientific and economic inequalities in developed and developing countries" (Carillo \& Papagni, 2014 in de Beer, 2020, p 69). There is considerably less literature on these inequalities as "the majority of action and discussion on open science has been dominated by actors and institutions in the Global North... with less focus on underlying power structures that tend to determine who can or cannot participate in the knowledge production processes" (Hillyer et al., 2020, p. 357). While significant work has been done by the International Development Research Centre (IDRC), future areas of critical OS research could prioritize the unique concerns of marginalized and developing countries in the Global South to further understand the potential for OS to rectify - or exacerbate - inequalities on an international scale. In other words, just because science is OS it does not mean that the process is more democratic, equitable and inclusive, as that requires additional attention to power, policies, practices, economics, and infrastructures, as well as capacity building as demonstrated in this thesis. IKDG and OS are commensurable if and only if Indigenous knowledge and data governance are included by design, in the same way that openness is included by design.

\subsection{Reflection}

This research is far from exhaustive. There are many contingencies related to sciencebased research in federally funded research, and it is not possible to make universal conclusions on best practices for Indigenous knowledge and data governance, but this did address a knowledge gap as just discussed. Also, OS differs for different scientific domain: best practices for social scientific research will not be best practices for geospatial mapping research, and each individual research project will yield unique questions, concerns, and types of knowledge/data, just as there is no one IKDG, it is community specific. In some cases, Indigenous data and 
knowledge should be controlled and governed specifically by the community who contributed it: health-related data, and data about specific communities that may be used in social science research is clearly sensitive and there is little dispute that it should be owned, controlled, and possessed by the communities to whom they belong. However, it may not always be clear to researchers when the knowledge they are drawing from, or including, belongs to Indigenous peoples. Likewise, there is further ambiguity when it relates to, for instance, satellite images of Inuit territory in the Arctic. Does this knowledge belong to the community, and should it be exempt from research that will be open by default? Should these images be owned by the community?

These type of questions of what constituted Indigenous data frequently arose during the research process, and for the moment, I do not have an answer of what does and what does not constitute Indigenous data. While Elder Gwen Philips and the FNIGC might argue that anything pertaining to First Nations, Métis, and Inuit peoples should remain in their sole custody and used at their sole discretion, researchers who undertake studies in Indigenous territories, or focus on biodiversity in a way that overlap with Indigenous spaces or areas of knowledge, may disagree.

The answer, I believe, is not simply a more robust or clear definition of 'Indigenous knowledge' and 'Indigenous data'. In fact, the literature is fraught with various overlapping, but slightly dissimilar, definitions of TK, TEK, [etc.] which make it difficult to fully grasp the concept as it pertains to a specific research project. It could also be argued that the ambiguity of the parameters of Indigenous knowledge and data may deter researchers from properly and meaningfully approaching their use, as they may still fail to appropriately engage with these.

The goal of this work was not to offer a prescriptive solution, or even a generalizable consensus on the compatibility of OS and IKDG. If anything, the aim of this thesis was to 
consider the advantages and disadvantages of OS. As the Government of Canada continues to acknowledge and prioritize the Indigenous right to knowledge, data governance, Indigenous sovereignty, and reconciliation, many questions remain about the ways in which the OS can benefit or hinder these new priorities. This thesis is but one steppingstone along the path to understanding these issues.

While I am in no position to speak on behalf of any cultural group or community, I feel confident in saying that based on this study, the analysis of the organizational literature supporting and describing Indigenous knowledge and data governance, and the case study I conducted, that it is important that any research using Indigenous knowledge, data, and information, as well as any research that involves Indigenous peoples or communities, necessitates an in-depth discussion with relevant Indigenous communities regarding ethical standards, research expectations, and processes of knowledge and data governance. While each individual research study may differ, and therefore draw upon different forms of knowledge, data, and/or media, it is my understanding that knowledge governance, as an inherent right of Indigenous peoples in Canada, includes the steadfast co-development of research projects that will involve any of these elements. As each Indigenous group has indicated in the literature I examined, there is an abundance of irrelevant and potentially harmful research coming out of the Indigenous homelands which does not reflect the needs and priorities of Indigenous communities in those homelands, it will be important that OS research, moving forward, appropriately reflects these need and priorities more substantively. 


\subsection{Conclusion}

With this thesis I sought to understand how epistemologically, theoretically, and practically, Indigenous knowledge frameworks are the same and differ from Western scientific knowledge frameworks and methods. Also, Indigenous knowledge, data, and information are managed, used, governed, and disseminated in vastly different ways than they are Western knowledge producing institutions. Furthermore, I discovered that organizations, communities, and individuals, to move mutually and beneficially move forward in a post-colonial open science framework, more knowledge is required. For example, organizations representing Indigenous communities, such as the First Nations Information Governance Centre (FNIGC), or Inuit Tapiirit Kanatami (ITK), or the Métis National Council do not always have the requisite information with which to make informed decisions about implementing OS.

My research suggests that $\mathrm{OS}$ is a promising movement that has the potential to be more inclusive, beneficial, and diverse than the more traditional Western models of science which have often resulted in scientific research that lacks credibility, is inaccessible to many, and primarily includes Western voices.

I suggest that for Canada's OS movement to be truly postcolonial, and inclusive of Indigenous Peoples' needs, interests, and values, the process should be co-developed in a way that prioritizes Indigenous knowledge and data governance by design, in the same way that it facilitates open by design. There are various considerations and protocols that should be taken into account each time a research project is done collaboratively with First Nations, Métis, and/or Inuit partners: for instance, the Métis Principles for Ethical Research, the principles included in the National Inuit Strategy on Research, and the OCAP Principles, and there may also be community-specific protocols and expectations in place depending on the specific community 
involved. However, I believe that developing a framework for OS research that puts the governance of Indigenous knowledge and data in the hands of those who contribute it during codeveloped research, would better align with the priorities of Indigenous sovereignty. In addition, developing this 'new' science in this way could better align with the Government of Canada's intentions to build better relationships with Indigenous Peoples and offer a significant positive step towards the goal of reconciliation. Allowing Indigenous partners, the chance to exercise their right to knowledge and data governance, and ensuring that they are the ones to set the parameters for knowledge and data sharing and management, could be an effective way to codevelop a more inclusive and postcolonial open science. 


\section{Appendix 1. Ethics Application Package}

\section{A. Research Ethics Protocol Form}

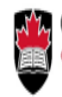 \\ Office of Research Ethics \\ CARLETON UNVERSTIY \\ Research Ethics Protocol Form
}

This is the primary CUREB study submission form, to be used when none of the other submission forms, intended for more specialized categories of research, re suitable. If you have any doubt about which form to use, of for help in completing this form, please contact the Office of Research Ethics at ethics Q carleton.ç or by phone: 6135202600 ext. 2517 (CUREB A) or ext. 4085 (CUREBB).

- Please submit this form as a new application in CuResearch. II this form is to replace a Release of Funds, it thould be submitted as an "Event" in CuResearch under the same study file. Pleases see our CuResearch User Manual for directions on how to submit n new application or an event.

- Note that all of our forms are compotible with Nicrosoft Office. Students and staff members can download a free copy of MS Office at no charge: Students: hittps://carleton.co/its/ms-offer-students/; Staff//Faculty: httoss//carleton.ca/its/allservices/computers/site-licensed-sottware/ms-ofier-focultyl

\begin{tabular}{ll} 
1. Tittle and Date & \\
1A Project Title & Title of Research Project (Detailed instructions, Example) \\
\hline $\begin{array}{l}\text { Co-developing Openness: Do open science and Indigenous praxis } \\
\text { alian? }\end{array}$ \\
\hline
\end{tabular}

1B Submission Date Date of completion of this form. Update each time the form is revised. (Detailed instructions, Example)

$$
\text { 2021-01-16 }
$$

\section{C Attachments List documents induded with this application (e.g. consent} materials, invitations, permissions) (Detailed instructions, Example)

\begin{tabular}{ll|}
\hline Appendices includes: Protocol Checklist, Email Invitation, \\
Informed Consent Form, Interview Guide, TCPS2 Certificate, and \\
Supervisor Signature Form
\end{tabular}

2A Lead Researche

Last name/First name

\begin{tabular}{|c|c|}
\hline (7) Academic or Library Staff & Hunter/Amanda \\
\hline Post-ddctoral Fellow & Official university (or other institution) email address \\
\hline 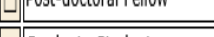 & amandahunter@cmail.carleton.ca \\
\hline$\nabla \mid$ Graduate Student & Department, faculty and institution (Detailed instructions, \\
\hline Undergraduate & Example) \\
\hline (1) Other & $\begin{array}{l}\text { School of Journalism and Communication, Faculty of Public Affairs } \\
\text { Carleton University }\end{array}$ \\
\hline
\end{tabular}

2B Academic Supervisor

Academic supervisor(s) Last name/First name. (Note, the

- Same as lead researcher

supervisor must be copied on all correspondence with CUREB.)

\begin{tabular}{l}
\hline Lauriault/Tracey $P$. \\
official university (or other institution) email address:
\end{tabular}

Form Version: August 2020

\section{tracey.lauriault@ecarleton.ca}

Department, faculty and institution (Detalled instructions,

Example)

School of Journalism and Communication, Faculty of Public Affairs, at Carleton University

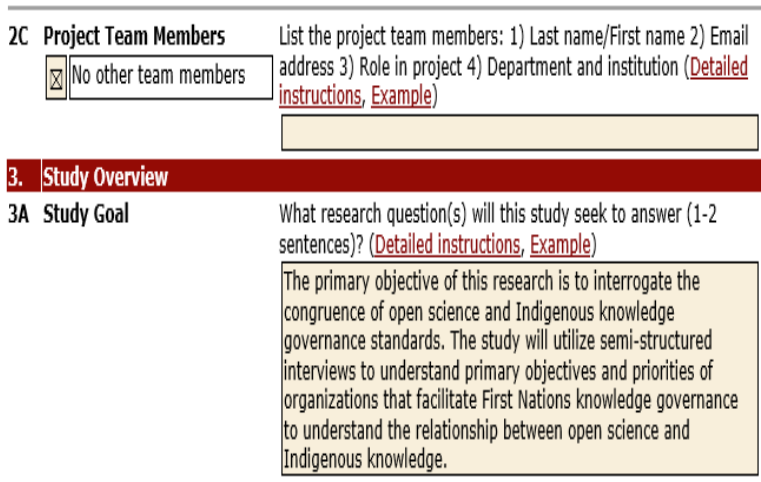

3B Study Purpose and Benefits Study rationale: why should the research be pursued; what are the benefits, and to whom? (Benefits can be to research community, companies, or society in general.) (Detailed instructions, Example)

This study seeks to address the gap in the discussion about open science adoption in Canada. The open science movement, and the standards which support it, are not compared and analyzed according to the principles and epistemological roots of First Nations, Inuit and Metis forms of knowledge and information governance. It is currently unclear to many (induding the First Nations Information Governance Centre) which aspects of open science could provide benefit for Indigenous researchers, and which concepts do not conceptually align with Indigenous knowledge systems. Thus, this research could provide guidance in this capacity and benefit First Nations, Inuit, and Metis interests in Canada. This research could also provide benefit for Indigenous and non-Indigenous researchers/organizations whose work is focused on the data collection, data management practices, standards, and methods of dissemination of open science and traditional Indigenous knowledge. Finally, this research could benefit policymakers and government departments whose work is focused on the adoption of open science in Canada.

3C Participant Interactions Overview

Briefly describe what will happen to, or will be required of, the participants during the research. (Only a project overview is required). (Detailed instructions, Example)

Participants of this research will be asked to participate in a semistructured interview conducted online via Zoom, which will consists of a number of open-ended questions from their expert professional perspective about their organization's work on Indigenous knowledge governance and data management frameworks. With the informed consent of participants, the 
researcher will be audio/video recording the interview and transcribing the interview electronically.

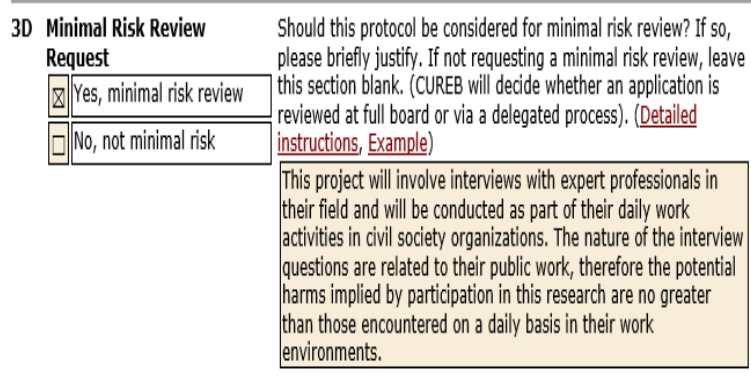

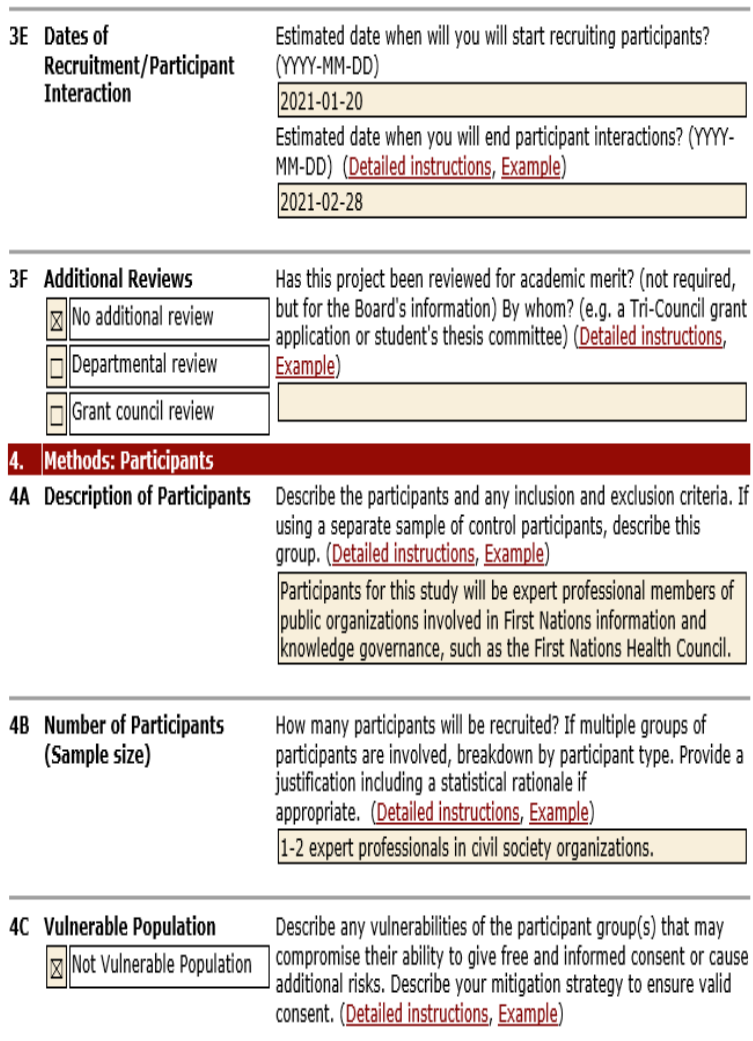

Form Version: August 2020
$3 / 14$
4D Participant Relationship to Describe any relationship that exists between the participants and Researcher the research team or any recruiting party or sponsor. Indicate how 8 No previous relationship relationships will be managed so there is no undue pressure on

\begin{tabular}{|l|l|}
\hline Z & No previous relationship \\
\hline \hline & Instructor-Student \\
\hline \hline & Client \\
\hline \hline & Employee \\
\hline \hline & Friends/Family \\
\hline \hline & Participated in previous \\
\hline \hline & study \\
\hline \hline & Other \\
\hline
\end{tabular}

4E Benefits to Participants Describe any potential direct benefits to the research participants $\square$ No Direct Benefits as opposed to society or knowledge. (Detailed instructions,

This study could benefit the participants as they are expert professionals in the field of Indigenous knowledge governance who are unsure of the benefit of adopting an open science praxis. This study will offer a critical analysis of open science standards and methods and demonstrate ways that they do/do not benefit Indigenous communities and researchers in Canada. The thesis and any thesis publications will be shared electronically with participants upon their completion.

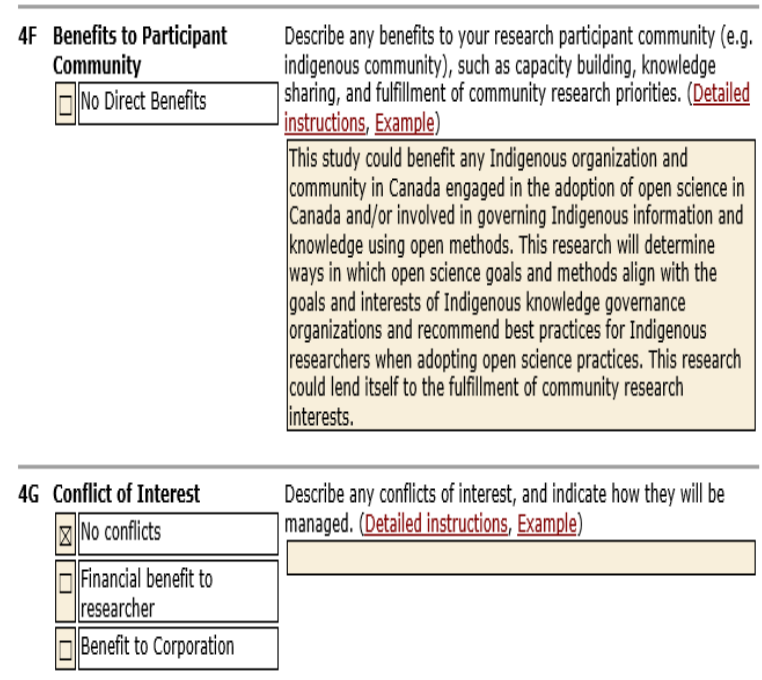

Form Version: August 2020 


$$
\square \text { other }
$$

4H Researcher Training with Participant Group

- Not applicable

addition to the TCPS2 training, describe any additional training the researcher(s) have (or will receive) to work with the proposed participants (e.g. research with Indigenous communities). (Detailed instructions, Example, TCPS2 Training)

In addition to the TCPS2 training, the researcher has completed the Fundamentals of OCAPQ online training course offered by the First Nations Information Governance Centre through Algonquin Colleae.

Indigenous Peoples and Community Engagement

5A Research involving Indigenous/Aboriginal peoples

If none of the statements are applicable, skip this section (Detailed instructions, Example)

7 Recruitment criteria includes Indigenous identity as a significant factor

7 Study will seek input from participants regarding Indigenous communities, cultures, artifacts, traditional knowledge or unique characteristics

7 Indigenous identity or membership in an Indigenous community is a factor in data analysis (e.g. sub-group analysis)

8 Interpretation of the research findings will refer to Indigenous communities, peoples, languages, histories or cultures

\begin{tabular}{l} 
5B Consultation \\
Describe the consultation process with the indigenous \\
community/lies. What is the community's involvement in \\
governance of the research? With whom did you consult and what \\
arrangements, if any, were made to implement Tri-Council (TCPS 2 \\
Chapter 9) principles? If no consultation has taken place, please \\
explain. (Detalled instructions, Example) \\
$\begin{array}{l}\text { The nature of the research is to determine the potential benefit of } \\
\text { open science methods for Indigenous researchers and scientists } \\
\text { from the perspective of Indigenous knowledge governance. The } \\
\text { research will not make any assumptions on behalf of Indigenous } \\
\text { communities or solicit any personal input from the } \\
\text { community/individual member level. No personal information will } \\
\text { be collected about any community or individual, so consultation } \\
\text { will not be necessary. } \\
\text { This research will involve interviews only with expert professionals } \\
\text { in the field of Indigenous information governance to understand } \\
\text { the goals and approaches of their professional organizations and } \\
\text { questions will only be asked about their daily work. The object of } \\
\text { analysis is the knowledge framework(s) applied by their } \\
\text { organizations to collect, manage, and disseminate information } \\
\text { and data, not the people, communities, or organizations } \\
\text { themselves. } \\
\text { The expert(s) will be consulted on the use of their information and } \\
\text { quotes for the final thesis. }\end{array}$ \\
\hline
\end{tabular}

5C Approvals/Agreements As part of the above process, describe what approvals/agreements you have made with the participating community/ies. (Detalled instructions, Example)

$$
N / A
$$

5D Benefits to Participant

Describe how the research will provide fair benefits to the

Community participating community/ies, meet community research priorities, support capacity building through enhancement of the skills of community personnel, and recognize the role of elders and other knowledge holders. (Detailed instructions, Example)

The objective of this research is to determine whether or not open science praxis aligns with the goals and objectives of Indigenous science and knowledge governance. This is an understudied topic, and important as Canada continues to adopt an open science framework. This research could be extremely beneficial for those communities and organizations who are unsure of when/how open science methods could benefit or disadvantage their traditional and scientific knowledge governance, especially for those communities and organizations looking for guidance on a set of best practices. Thus, the goal of this research is to benefit Indigenous communities and organizations by providing and sharing this analysis to further community research priorities and inform future skill building.

The research will also consider how open infrastructures interact with traditional knowledge, considering when (and if) openness is beneficial for the dissemination and preservation of traditiona knowledge. This approach will rely on foundational traditional knowledge as an object of inquiry, however it will not exploit or share specific iterations of this traditional knowledge.

5E Participant involvement in Describe how participants will be given the opportunity to research finding participate in the interpretation of the data and review of research findings prior to the completion of any reports or publications? If such participation will not occur, explain. (Detailed instructions, Example)

Upon completion of a final draft of the research, I will contact all participants and ask them to review the findings and confirm the permission to use their quotes and information. If the draft is not permissible, participants can contact me by email about changes until February 28th, 2021. All subsequent thesis publications will require approval from participants prior to publication.

5F Data Ownership, Control. Describe arrangements for the participating community's/ies' Access and Possession ownership and/or sharing of project data and findings, including the OCAP principles (ownership, control, access and possession). The information collected for this research will not be related to any Indigenous communities or individuals; rather, the qualitative data gathered will be related to the knowledge governance frameworks and goals of expert professionals and civil society organizations. This information will be audio/video recorded and

Form Version: August 2020 


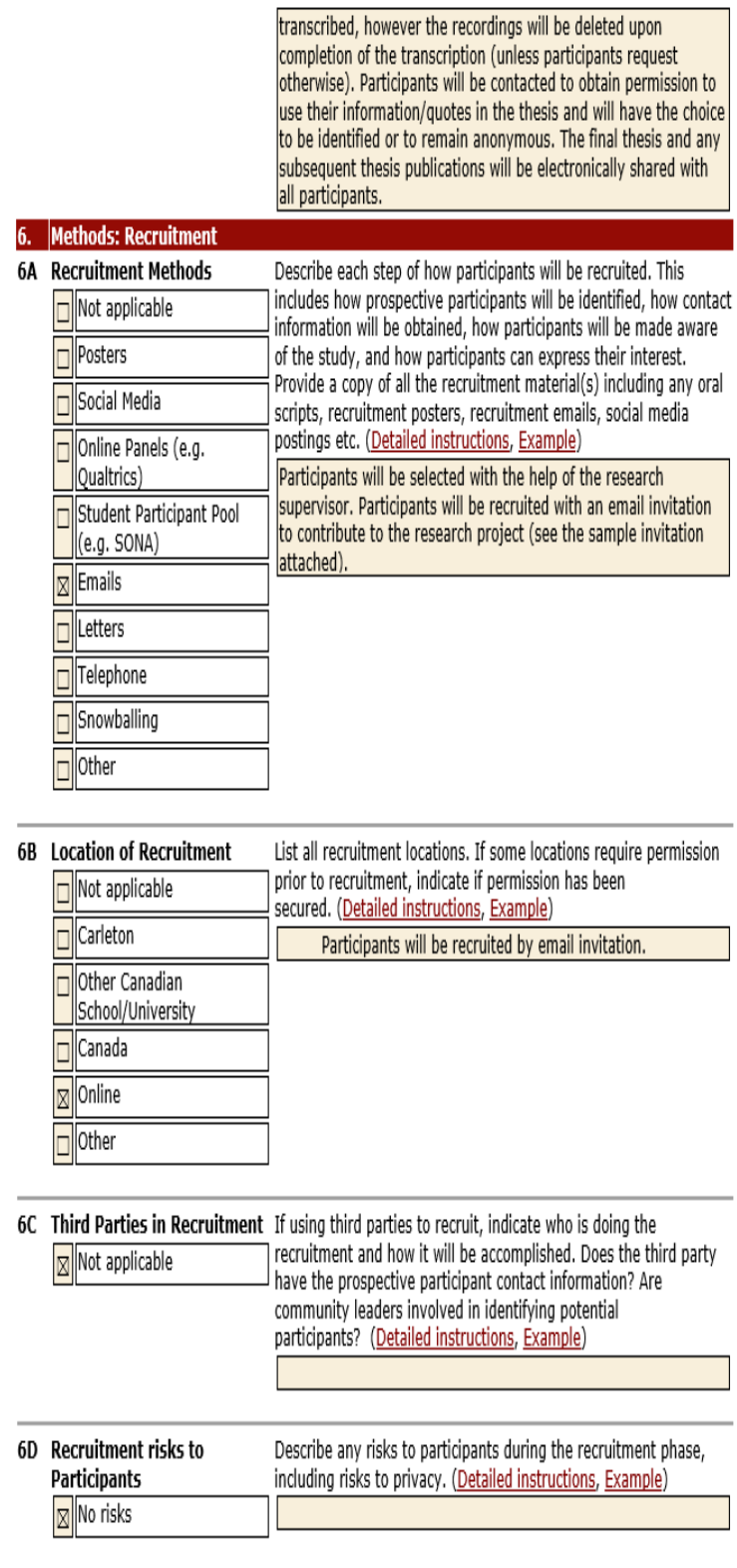

Form Version: August 2020

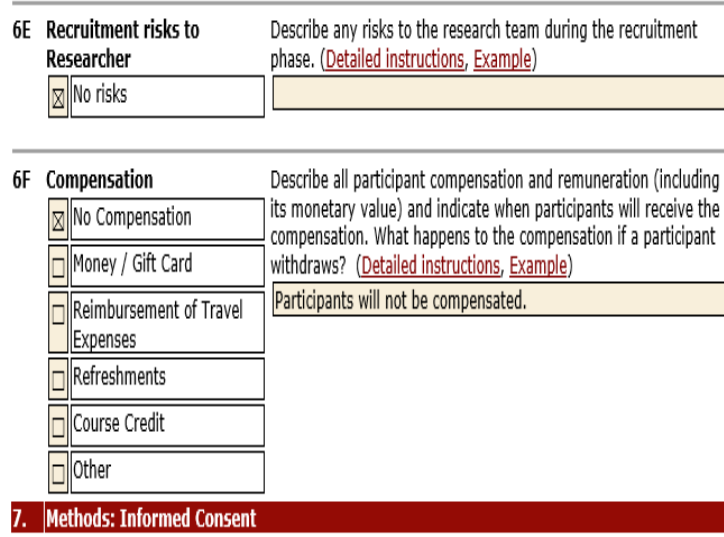

7A Obtaining informed consent Describe the process for obtaining informed consent from the 8 Signed consent participants (or guardians/legal representatives). If written consent is not used, explain the alternative method chosen. Include a copy 7 Online consent of all consent forms, scripts and other materials. (Detailed (- Oral consent instructions, Example)

Written and informed consent will be obtained by providing 7 Implied consent a consent form and detailed description of the research with the 7 Parent/Guardian consent email invitation to participate. Prospective participants can freely 9 Parent/Guardian consent $\begin{aligned} & \text { emase whether or not to partake in the interview process (form } \\ & \text { choose }\end{aligned}$ 7. Assent attached).

(7) 0 Other

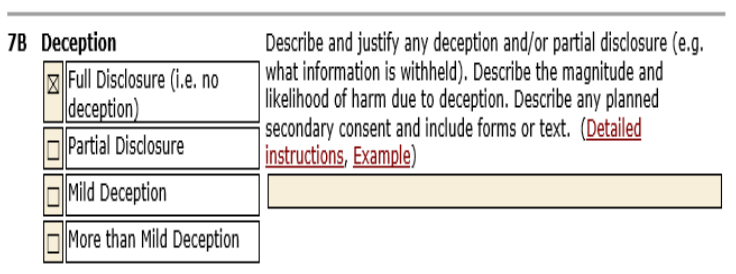

\begin{tabular}{l}
\hline 7C Debriefing \\
$\begin{array}{ll}\text { Describe if, when, and how participants will be debriefed. (Include } \\
\text { Describe any risks during debriefing and how they will be } \\
\text { mitigated. (Detailed instructions, Example) }\end{array}$ \\
\hline
\end{tabular}

7D Withdrawal Procedures Describe the procedures for a participant to withdraw. What will 7 Not applicable happen to data from participants who withdraw? Describe any \begin{tabular}{|l|l|l}
\hline & deadlines and limitations on withdrawal, during the study or after \\
\hline$Z$ & Participants can withdraw
\end{tabular}

Form Version: August 2020 


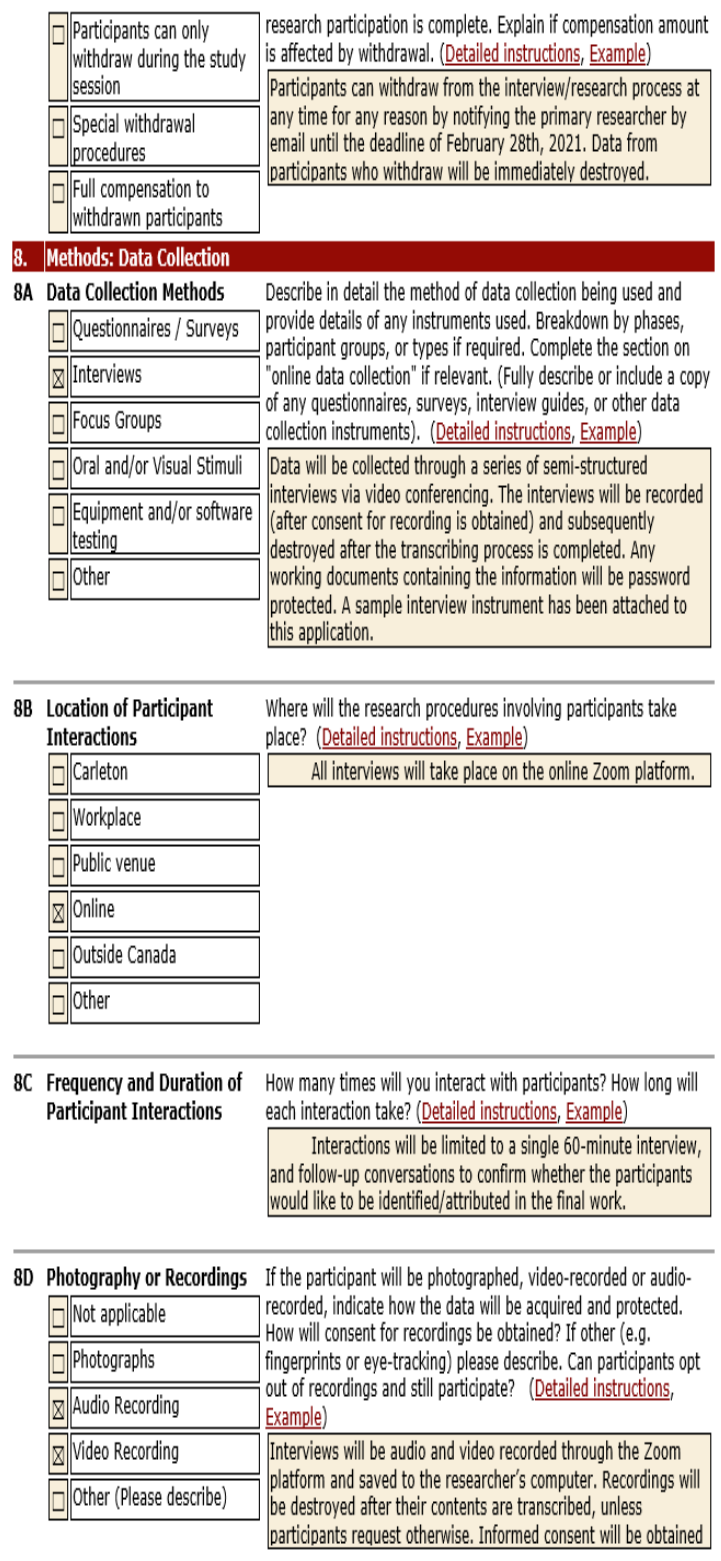

Form Version: August 2020 participants request otherwise. Informed consent will be obtained with the attached Informed Consent Form which will be sent to participants along with the email invitation to participate in the research. A signed consent form will be necessary in order to participate in the research.

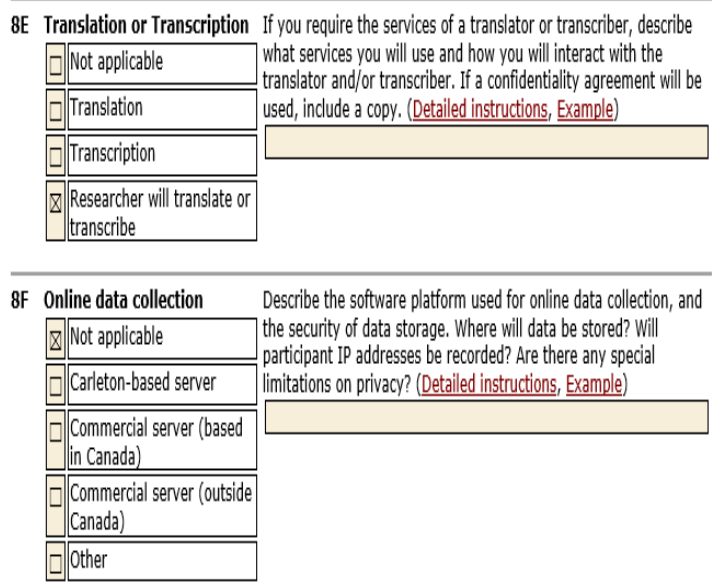

8G Biological specimens or Describe the apparatus and methods to collect biological specimens fluids Z Not applicable be stored? If any will be retained or transferred to another institution/research group, explain the research purpose, and plans for eventual destruction, if any. (Detailed instructions, Example)

\begin{tabular}{ll}
\hline fH Biological or physical & $\begin{array}{l}\text { Describe any drugs, devices or diagnostic apparatus being studied } \\
\text { interventions }\end{array}$ \\
$\begin{array}{ll}\text { or used, or any physical or physically intrusive research } \\
\text { interventions, such as sending energy into the body (e.g. } \\
\text { electrodes, MRI/X-ray), or physiological activities (e.g. exercise or } \\
\text { stress). Explain any risks to the participants and compare the dose } \\
\text { to established safety standards. (Detailed instructions, Example) }\end{array}$ \\
\hline
\end{tabular}

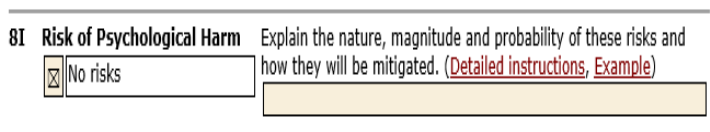

8] Risk of Physical Harm Explain the nature, magnitude and probability of these risks and
\begin{tabular}{|l|l|l|l} 
No risks & how they will be mitigated. (Detailed instructions, Example)
\end{tabular}


8K Risk of Social and/or Economic Harm

$$
8 \text { No risks }
$$

8L. Incidental Findings 8 Incidental findings unliker

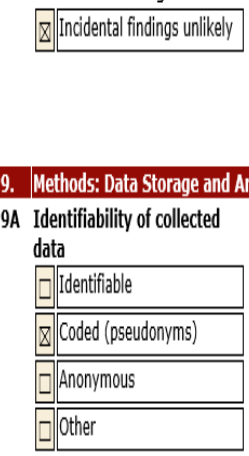

Describe possible incidental findings and how they will be managed (e.g. becoming aware of abuse of a child, imminent harm to a participant or third party, or potentially significant clinical findings). Any resulting limitations of confidentiality should be communicated to participants. (Detalied instructions, Example)

ysis

Describe the identifiability of research data at the point of data collection. If there are different levels of anonymity for different groups, describe. (Detailed instructions, Example)

The research data collected will pertain to the participants' expert professional work and the organizations with whom this work is performed. Information collected could therefore identiff participants. The interview recordings will be labelled and use a coding scheme to remove identifying information, and the code key will be stored separate from the data in the researcher's personal office. Participants will have the choice to be identified in the research or remain anonymous.

9B Identifiability of stored data Describe the identifiability of stored research data. If a link to

\begin{tabular}{|l|l|}
\hline$\square$ & Identifiable \\
\hline \hline & Coded (pseudonyms) \\
\hline \hline ] & Anonymous/anonymized \\
\hline \hline & Other \\
\hline
\end{tabular}
participant identities is retained (e.g. to permit compensation or withdrawal), also explain storage of linking data. Describe the process of anonymization if applicable. (Detaled instructions, Example)

The stored research data will contain data that could identify the participants. Transcriptions and physical notes from the interviews will be assigned codes to remove any personally identifying information and will be stored securely. The code key will be stored separately.

9 Identifiability of published data

\begin{tabular}{|c|c|}
\hline 8 Anonymous & $\begin{array}{l}\text { are different levels of anonymity for different groups, describe each } \\
\text { level here. (Detailed instructions, Example) }\end{array}$ \\
\hline ] Aggregate data only & \multirow{4}{*}{$\begin{array}{l}\text { Participants will be anonymous by default in the thesis and } \\
\text { any thesis publications unless otherwise requested. If they would } \\
\text { like to be identified, real participant names/data will be } \\
\text { attributed. }\end{array}$} \\
\hline Pseudonyms/Coded & \\
\hline \begin{tabular}{|l|l} 
Real participant names with \\
data attributable
\end{tabular} & \\
\hline 7 Other & \\
\hline
\end{tabular}

9D Data Storage (during the How will data be stored and kept safe? Provide details for each project) How will data be stored and kept safe? Provide details for each
type of data (e.g. raw data, contact lists, consent documents, - Encrypted

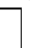

Form Version: August 2020

\begin{tabular}{|c|c|}
\hline Z 1 Password-protected & $\begin{array}{l}\text { anonymized data, recordings and images, electronic data and } \\
\text { paper documents). (Detailed instructions, Example) }\end{array}$ \\
\hline Z Physical documents & Research data will be stored on a password protected \\
\hline ] Other & $\begin{array}{l}\text { laptop computer which uses a virtul private network for added } \\
\text { protection. Physical data (if witten notes are collected) will be } \\
\text { stored in a locked fliling cabinet in the primary researcher's home } \\
\text { office. Data will only be accessible to the researcher and research } \\
\text { supervisor. }\end{array}$ \\
\hline
\end{tabular}

9E Data Disposition (after the After project completion, describe whether and how the data will project) be stored for future use. If shared, with whom? If made public, 0 how (e.g. online)? If archived, provide details. Describe any restrictions on access. Will personal identifiers be deleted and - De--dentifed data shared when? If data will be destroyed, when? Will participant contact publicly $\quad$ information be kept for future recruitment? (Include data IIdentifiable data shared disposition plans in the consent materials) (Detailed instructions, - Exublidy
Example)

1 All identifiers/codes will be Upon completing the project, the researcher will promptly destroy permanently deleted all data from participant interviews unless participants would like 10 Returned to participants to retain a copy. Contact information for participants will be kept in the event that further correspondence is needed (to obtain

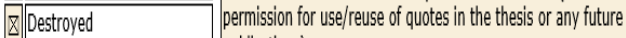
publications).

\begin{tabular}{ll}
\hline 9F Sharing Study Results & \multicolumn{1}{c}{ Do you intend to share a report (or summary) of the research } \\
\begin{tabular}{|l|l|}
\hline Results will be shared & findings with participants once the study is complete? If yes, \\
indude this option in the consent form. (Detalled instructions,
\end{tabular} \\
& \begin{tabular}{ll} 
Examole) \\
\hline
\end{tabular} \\
& $\begin{array}{l}\text { The thesis (and any subsequent publications using the research } \\
\text { findings) will be shared electronically with all participants and } \\
\text { their organizations. }\end{array}$ \\
\hline
\end{tabular}

\begin{tabular}{l}
\hline 9G Data Breach Risks \\
\begin{tabular}{|l|l|}
\hline No Risks & Describe the likelihood of a data breach and the resulting risks to \\
mitigated? (Detailed instructions, Example)
\end{tabular} \\
$\begin{array}{l}\text { There are no risks of data breach for this research. All data will be } \\
\text { securely stored on the researcher's computer in the researcher's } \\
\text { home. }\end{array}$ \\
\hline 10. Funding and Approvals
\end{tabular}

\begin{tabular}{|l|l|l|}
\hline 10 Project Funding & Who is funding this project? If applicable, include the funding \\
\hline & source/agency/company, program, award name, and number (from \\
\hline & CuResearch). Note if the researcher applied for a release of funds \\
\hline & Tri-Council Funded & for this project funding. \\
\hline
\end{tabular}

Form Version: August 2020 


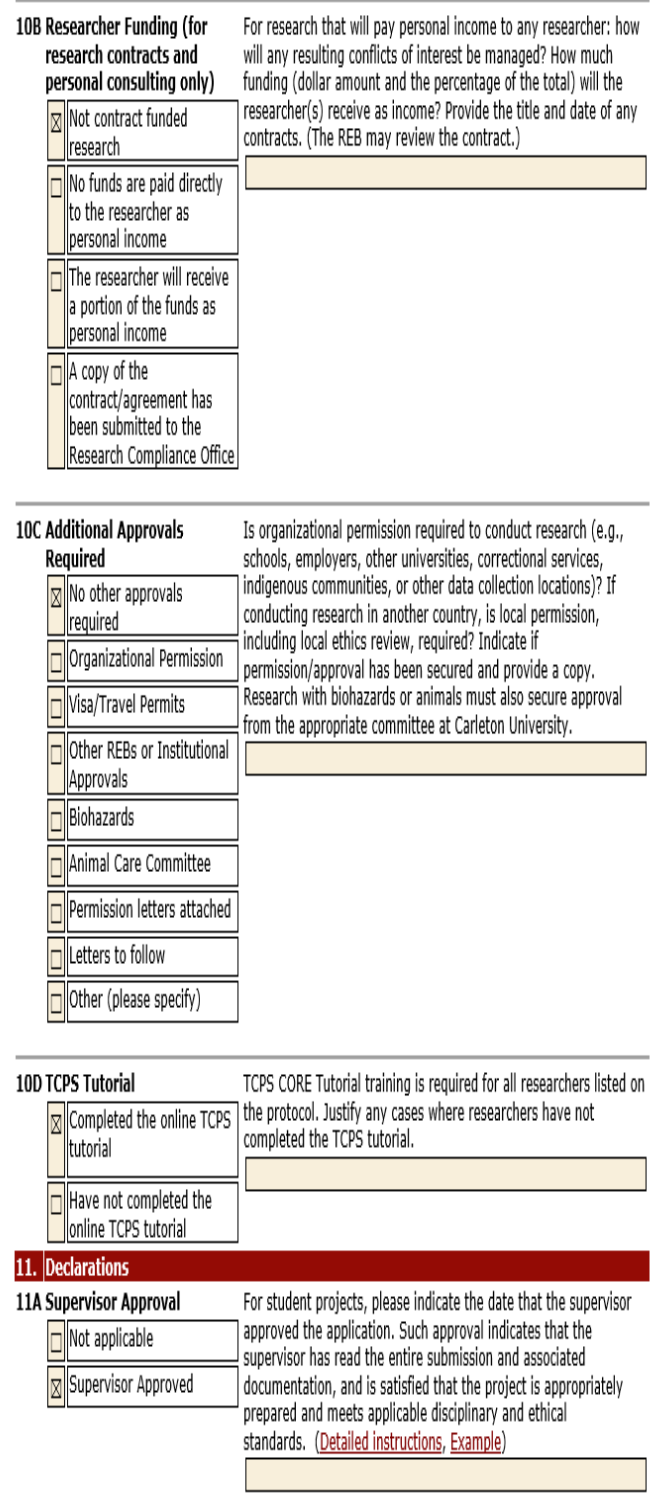

Form Version: August 2020

This ethics application accurately describes the research project or
sid Declaration \#1
Example)


1B. TCPS2 Core Certification

\section{PANEL ON \\ RESEARCH ETHICS \\ TCPS 2: CORE \\ Certificate of Completion}

This document certifies that

Amanda Hunter

has completed the Tri-Council Policy Statement: Ethical Conduct for Research Involving Humans Course on Research Ethics (TCPS 2: CORE) 


\section{Appendix 2. Semi-Structured Interview Description and Materials}

\section{A. Description}

On May $20^{\text {th }}, 2021$, I conducted a semi-structured interview with Elder Gwen Phillips of the Ktunaxa First Nation in British Columbia. 'Semi-structured' here means that I arrived at our interview time prepared with questions for Elder Gwen, but the conversation was primarily guided by her storytelling and insight about Indigenous knowledge and data governance as she understands and practices it. Elder Gwen and I met via Zoom for approximately one hour. Our meeting was recorded using the Zoom recording function, and our conversation was transcribed using the Otter AI automatic transcription software.

My questions were as follows, though we did not necessarily discuss them all:

- How did your work on data governance begin?

- What is the goal and purpose of effective First Nations data and information governance?

- In your own words, why is data governance important and what kind of impact does it have?

- Can you speak on some barriers to data governance - locally or more broadly - that you and your organization have encountered since your work started?

- What would you say are the priorities that guide your work?

- How do you and your organization conceive of open data?

- How important is knowledge sharing in your work?

- Is there traditional knowledge that shouldn't be shared outside of the communities who produce it? Is traditional knowledge sensitive knowledge? 


\section{B. Materials}

\section{Letter of Invitation}

Subject: Invitation to participate in a master's Thesis research study about Indigenous knowledge governance in open science practices

December 20th, 2020

Hello,

My name is Amanda Hunter, and I am a master's student in the School of Journalism and Communication at Carleton University. I am working on a research project under the supervision of Dr. Tracey P. Lauriault, Associate Professor of Critical Media and Big Dig Data.

I am writing to you today to invite you to participate in a research study focusing on open science in the Canadian Indigenous context. This study aims to investigate the advantages and disadvantages presented by the open science process on the practices of Indigenous knowledge governance.

This study involves a 60-minute semi-structured interview that will take place over the telephone or by video call. With your consent, interviews will be audio or video recorded, and I will transcribe the interview electronically. I will gladly share the transcription and the interview recording with you electronically. Once the recording has been transcribed and verified, the recording will be destroyed unless you wish to retain a copy.

While this project does not involve any foreseen professional and emotional risks, care will be taken to protect your identity. This will be done by keeping all responses anonymized and allowing you to request that certain responses not be included in the final project. You can be identified in the final product if you so choose.

You will have the right to skip the questions that you do not want to answer and end your participation in the study at any time, for any reason, up until January 31st, 2020. If you choose to withdraw, all the information you have provided will be destroyed.

No compensation will be provided for participation, but I would be happy to share my published thesis with you and your organization. The thesis and any other thesis publications will be shared electronically.

All research data, including audio/video recording and any notes will be encrypted. Any hard copies of data including handwritten notes will be kept in a locked filing cabinet in the researcher's private home office. Research data will only be accessible by the researcher and the research supervisor.

This research has been cleared by Carleton University Research Ethics Board A Clearance \#115200. Should you have any ethical concerns with the study, please contact the REB Chair, Carleton University Research Ethics Board-A (by phone: 613-520-2600 ext. 2517 or by email: ethics@carleton.ca). For all other questions about the study, please contact the researcher.

If you would like to participate in this research project, or have any questions about the research, please contact me at amandahunter@cmail.carleton.ca.

Sincerely, Amanda Hunter 


\section{Informed Consent Form}

Name and Contact Information of Researchers:

Amanda Hunter, Carleton University, Department of Journalism and Communication, Faculty of Public Affairs

Tel.:

Email:

Supervisor and Contact Information: Dr. Tracey P. Lauriault, Associate Professor of Critical Media and Big Data, Communication and Media Studies, School of Journalism and

Communication, Faculty of Public Affairs

Tel: 613-520-2600 ext. 7443

Email: tracey.lauriault@carleton.ca

Project Title

Asymmetrical Openness: Rethinking open science in the Canadian Indigenous context

Project Sponsor and Funder (if any)

$\mathrm{N} / \mathrm{A}$

\section{Carleton University Project Clearance}

Clearance \#: $115200 \quad$ Date of Clearance: February 17th 2021

\section{Invitation}

You are invited to take part in a research project because you are a respected professional expert in a recognized organization that facilitates the practice of Indigenous knowledge governance for First Nations, Metis, and/or Inuit peoples. The information in this form is intended to help you understand what we are asking of you so that you can decide whether you agree to participate in this study. Your participation in this study is voluntary, and a decision not to participate will not be used against you in any way. As you read this form, and decide whether to participate, please ask all the questions you might have, take whatever time you need, and consult with others as you wish.

\section{What is the purpose of the study?}

The primary objective of this research is to interrogate the congruence of open science and Indigenous knowledge governance standards. The study will utilize semi-structured interviews to understand primary objectives and priorities of organizations that facilitate Indigenous knowledge governance (First Nation, Metis, and Inuit) to understand the relationship between open science and Indigenous knowledge. This work is important because Canada is in the process of implementing open science mandates and directives to make the process of scientific research more open and accessible. While this has may benefits, there seems to be a lack of consideration paid to the potential implications of the open science framework on objectives of Indigenous knowledge governance. I will, therefore, address this research gap with this research study.

\section{What will I be asked to do?}

If you agree to take part in the study, we will ask you to participate in a one-on-one, semistructured interview of approximately 60 minutes. I wish to ask you some questions about the goals and objectives of your organization and its approach to Indigenous knowledge governance. 
By understanding the main priorities of your organization, I can critically analyze the standards and objectives of open science to identify the advantages and disadvantages that arise and make suggestions about how to develop more culturally specific standards for open science. This interview will take place by phone or video call, whichever you feel most comfortable with. The call will be audio/videotaped pending your consent, but you can freely choose not to be recorded. I will share the recording with you, the participant, if you wish. I will transcribe the recording into an electronic Microsoft Word document that will be shared between myself and the research supervisor, and can share the transcribed version with you, the participant, if you wish. After the interview is conducted and transcribed, I will reach out to you to confirm that the use of quotes in my thesis are acceptable and confirm whether you wish to be identified. If not, you will be anonymized in the research and can still revoke your consent until January 31st, 2020.

\section{Risks and Inconveniences}

We do not anticipate any foreseeable risks to participating in this study as we will not be asking for any personal information. The questions will be about the work done by your organization to facilitate Indigenous knowledge governance.

\section{Possible Benefits}

There are several potential benefits to this research and your participation in this study. As mentioned, it does not appear that research is being done to critically analyze the standards and goals of open science in comparison to the objectives and goals of Indigenous knowledge governance in Canada. Therefore, you may not receive any direct benefit from your participation in this study. However, your participation may allow researchers to better understand the ways in which open science standards can advantage and disadvantage Indigenous researchers, scientific output, and knowledge governance. It could also help inform a Canadian open science policy framework in the future, with the goal of addressing Indigenous-specific issues with open science practices. After the Master thesis is published, I would be happy to share an electronic copy with you, as well as any Thesis related publications.

\section{Compensation/Incentives}

You will not be paid or compensated for your participation in this study.

\section{No waiver of your rights}

By signing this form, you are not waiving any rights or releasing the researchers from any liability.

\section{Withdrawing from the study}

If you withdraw your consent during the study, all information collected from you before your withdrawal will be destroyed.

After the study, you may request that your data be removed from the study and deleted by notice given to the Principal Investigator (named above) by January 31st, 2020.

\section{Confidentiality}

If you choose not to be identified in the published thesis, we will remove all identifying information from the study data as soon as the interview has been transcribed and coded. I will also provide you with a copy of the transcribed interview for confirmation, as well as the original recording should you provide your consent to be recorded. 
We will treat your personal information as confidential, although absolute privacy cannot be guaranteed. No information that discloses your identity will be released or published without your specific consent. Research records may be accessed by the Carleton University Research Ethics Board to ensure continuing ethics compliance.

If the results of this study are published or presented at an academic conference or meeting, the data will be presented so that it will not be possible to identify any participants unless you give your express consent.

You will be assigned a code so that your identity will not be directly associated with the data you have provided. All data, including coded information, will be kept in an encrypted file on a secure computer. Your data will be stored and protected by the primary researcher.

For interviews conducted by video call, "in-session" data, such as the audio, video and chat transcript from the interview, will be stored locally on the researcher's computer. We will encrypt any research data that we store or transfer.

\section{Data Retention}

After the study has concluded your data (identified or de-identified) will be securely destroyed. As the participant, you can decide to not have the de-identified data destroyed if you wish to retain a copy.

\section{New information during the study}

If any changes could affect your decision to continue participating in this study, you will be promptly informed.

\section{Ethics review}

This project was reviewed and cleared by the Carleton University Research Ethics Board A. If you have any ethical concerns with the study, please contact Carleton University Research Ethics Board by phone at 613-520-2600 [ext. 2517] or by email at ethics@carleton.ca). 
After the study has concluded your data (identified or de-identified) will be securely destroyed unless you specify otherwise. As the participant, you can decide to not have the de-identified data destroyed if you wish to retain a copy.

\section{New information during the study}

In the event that any changes could affect your decision to continue participating in this study, you will be promptly informed.

\section{Ethics review}

This project was reviewed and cleared by the Carleton University Research Ethics Board A. If you have any ethical concerns with the study, please contact Carleton University Research Ethics Board (by phone at 613-520-2600 [ext. 2517] or by email at ethics@carleton.ca.

\section{Statement of consent - print and sign name}

I voluntarily agree to participate in this study.

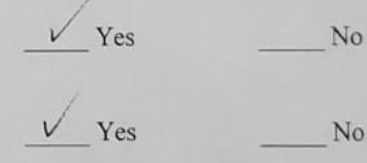

I agree to be audio/video recorded and understand that recordings will be destroyed after the interview data is transcribed.

I agree to the use of quotations (anonymized) in the published version of the thesis.

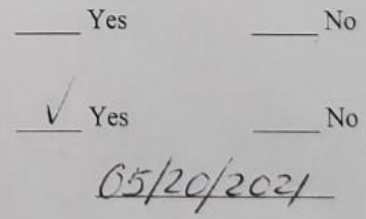

I agree to the use of quotations (identified) in the published version of the thesis.

\section{Gwen Phillips}

Signature
Date

Research team member who interacted with the participant

I have explained the study to the participant and answered any and all of their questions. The participant appeared to understand and agree. I provided a copy of the consent form to the participant for their reference.

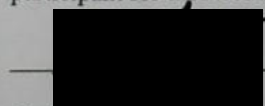

Signature of researcher
(Amanda Hunter)

May 8th, 2021

Date 


\section{References}

Adler, P. S. (2007). International Encyclopedia of Organization Studies. In International Encyclopedia of Organization Studies (Vol. 4, p. 1537). Sage Publications Inc. https://sk.sagepub.com/reference/organization/n530.xml

Akena, F. A. (2012). Critical Analysis of the Production of Western Knowledge and Its Implications for Indigenous Knowledge and Decolonization. Journal of Black Studies, 43(6), 599-619. https://doi.org/DOI: 10.1177/0021934712440448

Al Saba, R. (2020). Inequality and the impact of Covid-19: How discrimination is shaping the experiences of minorities and indigenous peoples during the pandemic. Minority Rights Group International. https://minorityrights.org/publications/covid-briefing/

Albornoz, D., Hillyer, B., Posada, A., Okune, A., \& Chan, L. (2019). Chapter 2: Principles for an Inclusive Open Science: The OCSDN Manifesto. In Contextualizing Openness: Situating Open Science. University of Ottawa Press.

Alexander, S. M., Provencher, J. F., Henri, D. A., Taylor, J. J., Lloren, J. I., Nanayakkara, L., Johnson, J. T., \& Cooke, S. J. (2019). Bridging Indigenous and science-based knowledge in coastal and marine research, monitoring, and management in Canada. Environmental Evidence, 8(36), 1-24. https://doi.org/10.1186/s13750-019-0181-3

Allen, C., \& Mehler, D. M. (2019). Open science challenges, benefits and tips in early career and beyond. PLOS Biology, 17(12), 1-14. https://doi.org/10.1371/journal.pbio.3000587

Anderson, Jane. E. (2015). Indigenous Knowledge and Intellectual Property Rights. International Encyclopedia of the Social \& Behavioral Sciences, 11, 769-778. http://dx.doi.org/10.1016/B978-0-08-097086-8.64078-3 
Armitage, D., \& Berkes, F. (2011). Co-management and the co-production of knowledge:

Learning to adapt in Canada's Arctic. Global Environnent Change, 21(3), 995-1004. https://doi.org/10.1016/j.gloenvcha.2011.04.006

Ashcroft, B., Griffiths, G., \& Tiffin, H. (2007). Post-Colonial Studies: The Key Concepts (Second). Routledge Taylor \& Francis Group. http://staff.uny.ac.id/sites/default/files/pendidikan/else-lilianissmhum/postcolonialstudiesthekeyconceptsroutledgekeyguides.pdf

Aspesi, C., \& Brand, A. (2020). In pursuit of open science, open access is not enough. Science, 368(6491), 574-577. https://doi.org/10.1126/science.aba3763

Baghramian, M., \& Carter, J. A. (2015). Relativism. The Stanford Encyclopedia of Philosophy, $1-60$.

Ball, M., Bloemers, M., Carr, D., Cavalli, V., Haglund, M., Kalaitzi, V., \& Vandevelde, K. (2020). A Vision for Open Science (pp. 1-7). http://doi.org/10.5281/zenodo.1491303

Barnhardt, R. \& Kawagley, O.A. (2005). Indigenous Knowledge Systems and Alaska Native Ways of Knowing. Anthropology \& Education Quarterly, 36(1), 8-23. https://doi.org/10.1525/AEQ.2005.36.1.008

Bartling, S., \& Friesike, S. (2014). Chapter 1: Towards Another Scientific Revolution. In Opening Science: The Evolving Guide on How the Internet is Changing Research, Collaboration and Scholarly Publishing (pp. 3-15). Springer Open. https://link.springer.com/content/pdf/10.1007\%2F978-3-319-00026-8.pdf

Battiste, M. (2002). Indigenous Knowledge and Pedagogy in First Nations Education: A Literature Review with Recommendations. Assembly of First Nations. 
https://www.afn.ca/uploads/files/education/24._2002_oct_marie_battiste_indigenouskno wledgeandpedagogy_lit_review_for_min_working_group.pdf

Battiste, M. (2005). Indigenous Knowledge: Foundations for First Nations. WINHEC:

International Journal of Indigenous Education Scholarship, 1, 1-17.

Battiste, M., \& Henderson, J. Y. (Eds.). (2000). Chapter 2: What is Indigenous Knowledge? In Protecting Indigenous Knowledge and Heritage: A Global Challenge (pp. 35-48). Purich Publishing Ltd.

https://books.google.ca/books?id=hXc9DAAAQBAJ\&printsec=frontcover\&source=gbs ge_summary_r\&cad $=0 \# \mathrm{v}=$ onepage $\& \mathrm{q} \& \mathrm{f}=$ false

Bell, J. (2018, March 27). The National Inuit Research Strategy: Five things you need to know. Nunatsiaq News. https://nunatsiaq.com/stories/article/65674the_national_inuit_research_strategy_five_thin gs_you_need_to_know/

Bengtsson, M. (2016). How to plan and perform a qualitative study using content analysis. NursingPlus Open, 2, 8-14. https://doi.org/10.1016/j.npls.2016.01.001

Berelson, B. (1952). Content analysis in communication research. Free Press.

Berkes, F. (1993). Traditional ecological knowledge in perspective. In Traditional Ecological Knowledge: Concepts and Cases (pp. 1-10). Canadian Museum of Nature/International Development Research Centre. https://library.um.edu.mo/ebooks/b10756577a.pdf

Berkes, F. (2010). Indigenous ways of knowing and the study of environmental change. Journal of the Royal Society of New Zealand, 39(4), 151-156.

https://doi.org/10.1080/03014220909510568 
Boot, G., \& Lowell, A. (2019). Acknowledging and Promoting Indigenous Knowledges, Paradigms, and Practices Within Health Literacy-Related Policy and Practice Documents Across Australia, Canada, and New Zealand. The International Indigenous Policy Journal, 10(3), 1-30. https://doi.org/DOI: 10.18584/iipj.2019.10.3.8133

Brandusescu, A., Iglesias, C., Lämmerhirt, D., \& Verhulst, S. (2019, February 20). Open data governance and open governance: Interplay or disconnect? Medium. https://anabrandusescu.medium.com/open-data-governance-and-open-governanceinterplay-or-disconnect-13d69a7bf7d0

British Columbia First Nations' Data Governance Initiative. (n.d.). About the BCFNDGI. BCFNDGI. https://www.bcfndgi.com/

Browne, T. D. (2012). Consent and Ethics on Local and Traditional Knowledge in the Internet Era: Some Key Questions [Thesis]. Carleton University.

Browne, T. D., \& Ljubicic, G. (2019). Chapter 8: Considerations for informed consent in the context of online, interactive, atlas creation. In Modern Cartography Series. DOI: 10.1016/B978-0-444-64193-9.00008-7

Cajete, G. (2000). Native Science: Natural Laws of Interdependence (First). Clear Light Publishers.

Canadian Internet Policy and Public Interest Clinic, \& Geomatics and Cartographic Research Centre. (2016). A Proposal: An Open Licensing Scheme for Traditional Knowledge. Canadian Internet Policy and Public Interest Clinic. https://cippic.ca/sites/default/files/file/CIPPIC_GCRC--TK_License_Proposal-July_2016.pdf

Carey, J. (1989). Communication as Culture (First). Unwin Hyman Inc. 
Carillo, M. R., \& Erasmo, P. (2014). "Little Science" and "Big Science": The institution of "Open Science" as a cause of scientific and economic inequalities among countries. Economic Modelling, 43, 42-56. http://dx.doi.org/10.1016/j.econmod.2014.06.021

Carpenter, T. (2012). Chapter 9: Electronic Publishing Standards. In Academic and Professional Publishing (1st ed., pp. 215-241). Chandos Publishing. https://www.sciencedirect.com/book/9781843346692/academic-and-professionalpublishing

Chakravartty, P., Kuo, R., Grubbs, V., \& McIlwain, C. (2018). \#CommunicationSoWhite. Journal of Communication, 68(2), 254-266. https://doi.org/10.1093/joc/jqy003

Chan, E., Bourgeois-Doyle, D., Donaldson, M., \& Haine-Bennett, E. (2020). Toward a UNESCO Recommendation on Open Science: Canadian Perspectives (p. 21).

Chan, L. (2019). Introduction: Situating Openness: Whose Open Science? In Contextualizing Openness: Situating Open Science (pp. 5-22). University of Ottawa Press.

Clogg, J. (2020, March 11). Colonialism is Alive and Well in Canada [Non-profit]. West Coast Environmental Law. https://www.wcel.org/blog/colonialism-alive-and-well-in-canada Coburn, E., Moreton-Robinson, A., Dei, G. S., \& Stewart-Harawira, M. (2013). Unspeakable Things: Indigenous Research and Social Science. Socio, 2, 121-134. https://doi.org/DOI:10.4000/socio.524

Couldry, N. \& Mejias, U.A. (2018). Data Colonialism: Rethinking Big Data's Relation to the Contemporary Subject. Television \& New Media, 20(4), 336-349. https://doi.org/10.1177/1527476418796632 
CODATA. (2020). Open Science for a Global Transformation: CODATA-coordinated submission to the UNESCO Open Science Consultation. Chemistry International, 42(4), 32. https://doi.org/10.1515/ci-2020-0412

Cramer, B. W. (2014). Review: To Save Everything, Click Here: The Folly of Technological Solutionism by Evgeny Morozov. Journal of Information Policy, 4, 173-175. https://doi.org/10.5325/jinfopoli.4.2014.0173

Cull, I., Hancock, R. L. A., McKeown, S., Pidgeon, M., \& Vedan, A. (2018). Pulling Together: A Guide for Front-Line Staff, Student Services, and Advisors. BCcampus. https://opentextbc.ca/indigenizationfrontlineworkers/

Dalton, C. M., Taylor, L., \& Thatcher, J. (2016). Critical Data Studies: A dialog on data and space. Big Data \& Society, 3(1), 1-9. https://doi.org/10.1177\%2F2053951716648346

Dalton, C. M., \& Thatcher, J. (2015). Inflated granularity: Spatial "Big Data" and geodemographics. Big Data \& Society, 2(2). https://doi.org/10.1177\%2F2053951715601144

David, P. A. (2008). The Historical Origins of "Open Science": An Essay on Patronage, Reputation and Common Agency Contracting in the Scientific Revolution. Capitalism \& Society, 3(2), 106. https://doi.org/10.2202/1932-0213.1040

de Beer, J. (2020). Open Innovation in Development: Integrating Theory and Practice Across Open Science, Open Access, and Open Data. In Making Open Development Inclusive: Lessons from IDRC Research (pp. 51-83). The MIT Press.

de la Fuente, G. B. (n.d.). What is Open Science? Introduction. FOSTER. https://www.fosteropenscience.eu/node/1420 
de Olivera, M.B. (2012). On the Commodification of Science: The Programmatic Dimension. Science \& Education (22), 2463-2483. DOI 10.1007/s11191-012-9455-7.

Deb Roy, R. (2018, September 4). Decolonise science - time to end another imperial era. The Conversation. https://www.smithsonianmag.com/science-nature/science-bearsfingerprints-colonialism-180968709/

Decolonizing Data: Indigenous Data Sovereignty Primer. (2017). Open North.

Delgado, R., \& Stefancic, J. (1998). Critical Race Theory: Past, Present, and Future. Current Legal Problems, 51(1), 467-491. https://doi.org/10.1093/clp/51.1.467

Drawson, A. S., Toombs, E., \& Mushquash, C. J. (2017). Indigenous Research Methods: A Systematic Review. The International Indigenous Policy Journal, 8(2), 1-25. https://doi.org/DOI: 10.18584/iipj.2017.8.2.5

Duran, E. (2006). Healing the Soul Wound: Counselling With American Indians and Other Native Peoples. Teacher's College Press. https://www.amazon.com/Healing-SoulWound-Multicultural-

Foundations/dp/B008W0QIJW?asin=0807746894\&revisionId=\&format=4\&depth=1

Edwards, P. (2004). A Vast Machine: Standards as Social Technology. Science, 304(5672), 827828. http://dx.doi.org/10.1126/science.1099290

Elam, J. D. (2019). Postcolonial Theory. Literary and Critical Theory. https://doi.org/DOI: 10.1093/OBO/9780190221911-0069

Elo, S., Kääriäinen, M., Kanste, O., Pölkki, T., Utriainen, K., \& Kyngäs, H. (2014). Qualitative Content Analysis: A Focus on Trustworthiness. SAGE Open, 4(1), 1-10. https://doi.org/10.1177\%2F2158244014522633 
Elo, S., \& Kyngäs, H. (2007). The qualitative content analysis process. Journal of Advanced Nursing, 62(1), 106-115. https://doi.org/DOI: 10.1111/j.1365-2648.2007.04569.x

Elo, S., \& Kyngäs, H. (2008). The qualitative content analysis process. Journal of Advanced Nursing, 62(1), 107-115. https://doi.org/10.1111/j.1365-2648.2007.04569.x

Engler, N., Scassa, T., \& Taylor, D. (2013). Legal Issues in Mapping Traditional Knowledge: Digital Cartography in the Canadian North. Cartographica: The International Journal for Geographic Information and Geovisualization, 48, 189-199. https://doi.org/10.3138/carto.48.3.1685

Etoroma, E. (2020). The Deep Roots of Anti-Black Institutional Racism in Canada. Social Science Humanities and Sustainability Research, 1(2), 65-74. https://doi.org/DOI: 10.22158/sshsr.v1n2p65

Fewer, D., Lauriault, T. P., \& Scassa, T. (2016). AN OPEN LICENSING SCHEME FOR TRADITIONAL KNOWLEDGE. Canadian Internet Policy and Public Interest Clinic. https://cippic.ca/en/TK_Open_Licensing_Proposal

First Nations Ethics Guide on Research and Aboriginal Traditional Knowledge. (2011). Assembly of First Nations. https://www.afn.ca/uploads/files/fn_ethics_guide_on_research_and_atk.pdf First Nations Information Governance Centre. (2014). Ownership, Control, Access and Possession $\left(O C A P^{T M}\right)$ : The Path to First Nations Information Governance (pp. 1-49). The First Nations Information Governance Centre. https://achh.ca/wpcontent/uploads/2018/07/OCAP_FNIGC.pdf

FNIGC. (2019). First Nations data sovereignty in Canada. Statistical Journal of the IAOS, 35(1), 47-69. https://doi.org/10.3233/SJI-180478 
FNIGC. (2020). A First Nations Data Governance Strategy (pp. 1-102). First Nations Information Governance Centre. https://fnigc.ca/wpcontent/uploads/2020/09/FNIGC_FNDGS_report_EN_FINAL.pdf

Ford, C. L., \& Airhihenbuwa, C. O. (2010). The public health critical race methodology: Praxis for antiracism research. Social Science \& Medicine, 71(8), 1390-1398. https://doi.org/10.1016/j. socscimed.2010.07.030

Frank, J. W., Moore, R. S., \& Ames, G. M. (2000). Historical and Cultural Roots of Drinking Problems Among American Indians. American Journal of Public Health, 90(3), 344-351. https://doi.org/doi: 10.2105/ajph.90.3.344

Fraser Taylor, D. R. (2013). Fifty Years of Cartography: Some Personal Reflections. The Cartographic Journal, 50(2), 187-191. https://doi.org/10.1179/0008704113Z.00000000087

Fraser Taylor, D. R. (2019). Chapter 1: Cybercartography Revisited. In Further Developments in the Theory and Practice of Cybercartography: International Dimensions and Language Mapping (Third, Vol. 7, pp. 3-23). Elsevier.

Friesike, S., Widenmayer, B., Gassmann, O., \& Schildhauer, T. (2014). Opening science: Towards an agenda of open science in academia and industry. The Journal of Technology Transfer, 40, 581-601. https://doi.org/10.1007/s10961-014-9375-6

Gagliardi, D., Cox, D., \& Li, Y. (2015). Chapter 4: Institutional Inertia and Barriers to the Adoption of Open Science. In The Transformation of University Institutional and Organizational Boundaries (pp. 107-133). SENSE Publishers. https://www.researchgate.net/publication/290433245_Institutional_Inertia_and_Barriers_ to_the_Adoption_of_Open_Science 
Gandhi, L. (1998). Postcolonial Theory: A Critical Introduction (First).

GCRC. (n.d.). Agreements of Cooperation and Membership. Geomatics and Cartographic Research Centre.

https://gcrc.carleton.ca/index.html?module=module.gcrcatlas_agreements_of_cooperatio n_and_membership

Gerard, D. G. (1941). The sociology of knowledge and the problem of truth. Journal of the History of Ideas, 2, 110-115.

Glaser, B. G., \& Strauss, A. L. (1967). The discovery of grounded theory: Strategies for qualitative research. Aldine Publishing.

Global Indigenous Data Alliance. (n.d.). CARE Principles for Indigenous Data Governance. Global Indigenous Data Alliance. https://static1.squarespace.com/static/5d3799de845604000199cd24/t/5da9f4479ecab221c e848fb2/1571419335217/CARE+Principles_One+Pagers+FINAL_Oct_17_2019.pdf

Government of Canada. (2016, May). Open by default and modern, easy to use formats.

Open.Canada.Ca. Open by default and modern, easy to use formats

Government of Canada. (2018). Canada's 2018-2020 National Action Plan on Open Government. Government of Canada, Open Government. https://open.canada.ca/en/content/canadas-2018-2020-national-action-plan-opengovernment

Government of Canada. (2019). Setting new directions to support Indigenous research and research training in Canada 2019-2022. Government of Canada. https://www.canada.ca/content/dam/crcc-ccrc/documents/strategic-plan-20192022/sirc_strategic_plan-eng.pdf 
Grahe, J. E., Cuccolo, K., Leighton, D. C., \& Cramblet Alvarez, L. D. (2020). Open Science Promotes Diverse, Just, and Sustainable Research and Educational Outcomes. Psychology Learning \& Teaching, 19(1), 5-20. https://doi.org/10.1177\%2F1475725719869164

Hall, D. M., \& Steiner, R. (2020). Policy content analysis: Qualitative method for analyzing subnational insect pollinator legislation. Methods $X, 7(6)$. https://doi.org/10.1016/j.mex.2020.100787

Hampson, G., Gadd, E., Vandegrift, M., Hinchliffe, L. J., Johnson, R., Erdmann, C., Steinhauer, J., \& DeSart, M. (2020). OSI Policy Perspective 3: Open Science Roadmap Recommendations to UNESCO. OSI Policy Perspectives, 1-91. https://doi.org/doi $10.13021 /$ osi2020.2735

Hanwell, M. (2018, December 29). What is open science? Left Review Online. https://leftreviewonline.com/english/science-technology/what-is-open-science.html Harley, J. B. (1989). Deconstructing the Map. Cartographica, 26(2), 1-20.

Hess, C., \& Ostrom, E. (2006). Introduction: An Overview of the Knowledge Commons. In Understanding Knowledge as a Commons - From Theory to Practice (pp. 3-26). https://mitpress.mit.edu/books/understanding-knowledge-commons

Hickey, C. (2020). Indigenous Epistemologies, Worldviews and Theories of Power. Turtle Island Journal of Indigenous Health, 1(1), 14-25. https://doi.org/10.33137/tijih.v1i1.34021 Hill Collins, Patricia. (n.d.). Toward a new vision: Race, class, and gender as categories of analysis and connection. In Race, gender, sexuality, and social class: Dimensions of inequality and identity. (2nd ed.). SAGE; Ares. 
Hillyer, R., Albornoz, D., Posada, A., Okune, A., \& Chan, L. (2020). Toward an Inclusive, Open, and Collaborative Science: Lessons from OCSDNet. In Making Open Development Inclusive: Lessons from IDRC Research (pp. 357-379). The MIT Press.

Hyett, S. L., Gabel, C., Marjerrison, S., \& Schwartz, L. (2019). Deficit-Based Indigenous Health Research and the Stereotyping of Indigenous Peoples. Canadian Journal of Bioethics, 2(9), 102-109. https://doi.org/10.7202/1065690ar

Impact Canada. (2018). Smart Cities Challenge Applicant Guide. Government of Canada. https://impact.canada.ca/en/challenges/smart-cities/applicant-guide

Indigenous Knowledge and Knowledge Transmission. (n.d.-a). UNESCO. https://en.unesco.org/links/transmission

Indigenous Knowledge and Knowledge Transmission. (n.d.-b). In UNESCO. https://en.unesco.org/links/transmission

Innovation, Science and Economic Development Canada. (2020, February 26). Roadmap for Open Science to reduce barriers and speed up discovery [Government of Canada]. Innovation, Science and Economic Development Canada. https://www.canada.ca/en/innovation-science-economicdevelopment/news/2020/02/roadmap-for-open-science-to-reduce-barriers-and-speed-updiscovery.html

Inuit Tapiirit Kanatami. (2018, March 22). National Inuit Strategy On Research. https://www.itk.ca/wp-content/uploads/2019/11/ITK_NISR_Roundtable_11.pdf Jeanette, H. W. (2008). Unmasking, Exposing, and Confronting: Critical Race Theory, Tribal Critical Race Theory and Multicultural Education. International Journal of Multicultural Education, 10(2), 1-15. http://dx.doi.org/10.18251/ijme.v10i2.137 
Kass, N. (2019, April 19). On the Ethics of Open Science. Sage Bionetworks. https://sagebionetworks.org/in-the-news/on-the-ethics-of-open-science-2/

Kerr, J. (2014). Western epistemic dominance and colonial structures: Considerations for thought and practice in programs of teacher education. Decolonization: Indigeneity, Education \& Society, 3(2), 83-104.

Kitchin, R. (2014). The data revolution: Big data, open data, data infrastructures and their consequences. Sage Publications. doi: 10.4135/9781473909472

Kitchin, R., \& Lauriault, T. P. (2014). Towards Critical Data Studies: Charting and Unpacking Data Assemblages and Their Work. In Thinking Big Data in Geography: New Regimes, New Research. University of Nebraska Press. https://flexpub.com/preview/thinking-bigdata-in-geography

Krippendorff, K. (2004). Content Analysis: An Introduction to Its Methodology. Sage Publications Inc.

Kukutai, T., \& John, T. (2016). Preface. In Indigenous Data Sovereignty: Towards an Agenda (pp. xxi-xxiii). Australian National University Press. https://library.oapen.org/bitstream/handle/20.500.12657/31875/624262.pdf?sequence=1\& isAllowed $=\mathrm{y}$

Lafuente, A. L., \& Estalella, A. (2015). Ways of science: Public, open, and commons. In Open science, open issues (pp. 27-57). UNIRIO.

Lane, A. (2013). Technological Solutionism. In Macmillan Dictionary. https://www.macmillandictionary.com/dictionary/british/solutionism 
Lasthiotakis, H., Kretz, A., \& Sá, C. (2015). Open science strategies in research policies: A comparative exploration of Canada, the US and the UK. Policy Futures in Education, 13(8), 968-989. https://doi.org/10.1177\%2F1478210315579983

Lauriault, T. P., \& Francoli, M. (2017). Openness, transparency, participation. In Understanding Spatial Media (pp. 188-203). Sage Publications Ltd. https://doi.org/10.1111/cag.12536

LeBel, E. P., Campbell, L., \& Loving, T. J. (2017). Benefits of Open and High-Powered Research Outweigh Costs. Journal of Personality and Social Psychology, 113(2), 230243. https://doi.org/10.1037/pspi0000049

Little Bear, L. (1999). Foreword. In Native Science: Natural Laws of Interdependence (First). Clear Light Publishers.

Little Bear, L. (2000). 5: Jagged Worldviews Colliding. In Reclaiming Indigenous Voice and Vision (pp. 77-85). UBC Press.

Little Bear, L. (2012). Traditional Knowledge and Humanities: A Perspective by a Blackfoot. Journal of Chinese Philosophy, 39(4), 518-527. https://doi.org/10.1111/j.15406253.2012.01742.x

Little Bear, L. (2009). Naturalizing Indigenous Knowledge: Synthesis Paper. Naturalizing Indigenous Knowledge, 1-28. https://www.afn.ca/uploads/files/education/21._2009_july_cclalkc_leroy_littlebear_naturalizing_indigenous_knowledge-report.pdf Loken, E., \& Gelman, A. (2017). Measurement error and the replication crisis. Science, 355(6325), 584-585. https://doi.org/10.1126/science.aal3618

Macnamara, J. (2018). 11: Content Analysis. In Mediated Communication (pp. 191-212). De Gruyter Mouton. https://doi.org/10.1515/9783110481129-012 
Magga, O. H. (2005). Indigenous Knowledge Systems-The True Roots of Humanism. Libraries - A Voyage of Discovery, 1-9. https://archive.ifla.org/IV/ifla71/papers/183e-Magga.pdf

Mannan, A. (2016). Science and Subjectivity: Understanding Objectivity of Scientific Knowledge. Philosophy and Progress, 59(1-2), 44-72. https://doi.org/DOI:http://dx.doi.org/10.3329/pp.v59i1-2.36680

Marshall, A., Bartlett, C., \& Marshall, M. (2012). Two-Eyed Seeing and other lessons learned within a co-learning journey of bringing together indigenous and mainstream knowledges and ways of knowing. Journal of Environmental Studies and Sciences, 2, 331-340. https://doi.org/10.1007/s13412-012-0086-8

Medelyan, A. (n.d.). Coding Qualitative Data: How to Code Qualitative Research (Updated 2021). Thematic. https://getthematic.com/insights/coding-qualitative-data/

Merricks White, J. (2019). Standardising the city: A material-discursive genealogy of CPAI_001, ISO 37120 and BSI PAS 181 [Doctoral]. Maynooth University.

Merrigan, G., Johnston, R. T., \& Huston, C. L. (2012). Communication Research Methods: Canadian Edition. Oxford University Press.

Morozov, E. (2013). To Save Everything, Click Here: The Folly of Technological Solutionism (First). Public Affairs. https://www.goodreads.com/book/show/13587160-to-saveeverything-click-here

Morris, A. (1978). Standards in Meteorology. In Air Quality Meteorology and Atmospheric Ozone (pp. 5-12). ASTM International. https://www.astm.org/DIGITAL_LIBRARY/STP/PAGES/STP36858S.htm Mosco, V. (2005). The Digital Sublime: Myth, Power, and Cyberspace. The MIT Press. https://books.google.ca/books?hl=en\&lr=\&id=cenuCwAAQBAJ\&oi=fnd\&pg=PR5\&dq= 
Mosco,+2005+technological+determinism\&ots $=$ Yz7 JdA2Az-

$\&$ sig=MW10VpMRJQaKdD8gaAycUiRhwNE\#v=onepage\&q\&f=false

Moss, L. (2003). Is Canada Postcolonial? Unsettling Canadian Literature. Wilfred Laurier

University Press. https://www.wlupress.wlu.ca/Books///Is-Canada-Postcolonial2

Mukurtu 100-Introduction and overview. (2020, December). Washington State University

Center for Digital Scholarship. https://mukurtu.org/support/mukurtu-introduction-andoverview/

Muller, S., Hemming, S., \& Rigney, D. (2019). Indigenous sovereignties: Relational ontologies and environnemental management. Geographical Research, 57(4), 399-410. https://doi.org/10.1111/1745-5871.12362

Ngcobo, K. M., \& Eyono Obono, S. D. (2013). Modeling ICT Adoption Factors for the Preservation of Indigenous Knowledge. World Academy of Science, Engineering and Technology International Journal of Information and Communication Engineering, 7(1), 223-228. https://doi.org/doi.org/10.5281/zenodo.1072104

Nielsen, M. (2011). Reinventing Discovery: The New Era of Networked Science. Princeton University Press.

Nunaliit Atlas Framework. (n.d.). Nunaliit by GCRC. http://nunaliit.org Nunavut Communities. (2019). Final Proposal: Katinnganiq: Community, Connectivity and Digital Access for Life Promotion in Nunavut. Infrastructure Canada. https://www.infrastructure.gc.ca/cities-villes/videos/nunavut-eng.html OECD. (2020). Why open science is critical to combatting COVID19. OECD. http://www.oecd.org/coronavirus/policy-responses/why-open-science-is-critical-tocombatting-covid-19-cd6ab2f9/ 
Oliver, T. (2010). Effects of Colonialism on Indigenous People in Canada. Simon Fraser University. http://manitobawildlands.org/pdfs/TonyOliver-BriefHistory_2010.pdf Pels, D. (1996). Karl Mannheim and the Sociology of Scientific Knowledge: Toward a New Agenda. Sociological Theory, 14(1), 30-48. https://doi.org/10.2307/202151

Peltier, C. (2018). An Application of Two-Eyed Seeing: Indigenous Research Methods With Participatory Action Research. International Journal of Qualitative Methods, 17(1), 112. https://doi.org/10.1177\%2F1609406918812346

Phillips, G. (2017). Indigenous Data Sovereignty and Reconciliation [Keynote Presentation]. Data Power 2017, Ottawa. https://www.youtube.com/watch?v=4I_3figC3B0\&ab_channel=DataPower Phillips, G. (2021, May). MA Thesis Interview [Video].

Piron, F. (2018). Postcolonial Open Access. In Open Divide? Critical Studies on Open Access. Litwin Books. https://corpus.ulaval.ca/jspui/handle/20.500.11794/16178

Posey, D. A., \& Dutfield, G. (1996). Beyond Intellectual Property: Toward Traditional Resource Rights for Indigenous Peoples and Local Communities. International Development Research Centre. https://books.google.ca/books?hl=en\&lr=\&id=HZJ6KPQBc_EC\&oi=fnd\&pg=PP12\&dq $=$ indigenou + open + science + traditional\&ots $=$ tEGrdXb0uc\&sig $=9$ PpryV4iyQh 8 ONF7OC wLbjyf $2 \mathrm{x} 0 \# \mathrm{v}=$ onepage $\& \mathrm{q}=$ indigenou $\% 20 \mathrm{open} \% 20$ science $\% 20$ traditional $\& \mathrm{f}=$ false Pulsifer, P. L., Laidler, G. J., Fraser Taylor, D.R., \& Hayes, A. (2011). Towards an Indigenist data management program: reflections on experiences developing an atlas of sea ice knowledge and use. Canadian Geographer, 55(1), 108-124. https://doi.org/10.1111/j.1541-0064.2010.00348.x 
Pulsifer, P. L., Huntington, H. P., \& Pecl, G. T. (2014). Introduction: Local and traditional knowledge and data management in the Arctic. Polar Geography, 37(1), 1-4. https://doi.org/10.1080/1088937X.2014.894591

Purdue Online Writing Lab. (n.d.). Critical Race Theory (1970s-present). Purdue University. https://owl.purdue.edu/owl/subject_specific_writing/writing_in_literature/literary_theory _and_schools_of_criticism/critical_race_theory.html

Radder, H. (2019). From Commodification to the Common Good: Reconstructing Science, Technology, and Society. 10.2307/j.ctvnb7qrh.

Rainie, S., Rodriguez-Lonebear, D., \& Martinez, A. (2017). 2017b. Policy Brief: Data Governance for Native Nation Rebuilding (Version 2). University of Arizona Native Nations Institute. http://nni.arizona.edu/application/files/8415/0007/5708/Policy_Brief_Data_Governance_ for_Native_Nation_Rebuilding_Version_2.pdf

Randall, D., \& Wesler, C. (2018). The Irreproducibility of Modern Science: Causes, Consequences, and the Road to Reform (pp. 11-16). National Association of Scholars. https://www.nas.org/storage/app/media/Reports/Irreproducibility\%20Crisis\%20Report/N AS_irreproducibilityReport.pdf

Renkewitz, F., \& Heene, M. (233 C.E.). The Replication Crisis and Open Science in Psychology. Zeitschrift Für Psychologie, 227(4), October 2019. https://doi.org/10.1027/21512604/a000389

Roberts, S. O., Bareket-Shavit, C., Dollins, F. A., Goldie, P. D., \& Mortenson, E. (2020). Racial Inequality in Psychological Research: Trends of the Past and Recommendations for the 
Future. Perspectives on Psychological Science, 15(6), 1295-1309.

https://doi.org/10.1177\%2F1745691620927709

Russell, A. L. (2014). Open Standards and the Digital Age: History, Ideology, and Networks. Cambridge University Press.

Russo Carroll, S., Garba, I., Figueroa-Rodríguez, O. L., Holbrook, J., Lovett, R., Materechera, S., Parsons, M., Raseroka, K., Rodriguez-Lonebear, D., Rowe, R., Sara, R., Walker, J. D., Anderson, J., \& Huson, M. (2020). The CARE Principles for Indigenous Data Governance. Data Science Journal, 19(1), 43-54. https://doi.org/DOI: http://doi.org/10.5334/dsj-2020-043

Scassa, T., Engler, N. J., \& Taylor, D. R. F. (2015). Legal Issues in Mapping Traditional Knowledge: Digital Cartography in the Canadian North. The Cartographic Journal, 52(1), 41-50. https://doi.org/10.1179/174327713X13847707305703

Schooler, J. W. (2014). Metascience could rescue the 'replication crisis.' Nature, 515(9), 9. https://doi.org/10.1038/515009a

Segal, H. P. (1986). The Technological Utopians. In Imagining Tomorrow: History, Technology and The American Future. The MIT Press. http://web.mit.edu/m-it/science_fiction/jenkins/jenkins_1.html

Sengupta, P. (2020). Open access publication: Academic colonialism or knowledge philanthropy? Geoforum, 118, 203-206. https://doi.org/10.1016/j.geoforum.2020.04.001

Shapin, S. (2015). Truth and Credibility in Science. In International Encyclopaedia of the Social \& Behavioural Sciences (2nd ed., pp. 673-678). Elsevier. https://www-sciencedirectcom.proxy.library.carleton.ca/science/article/pii/B9780080970868850394?via\%3Dihub 
Shoemaker, P. J., \& Reese, S. D. (1996). Mediating the Message: Theories of Influences on Mass Media Content (Second). Longman Publishers. http://chinhnghia.com/mediating-themessage.pdf

Shrout, P. E., \& Rodgers, J. L. (2018). Psychology's replication crisis as scientific opportunity: A précis for policymakers. Policy Insights from the Behavioral and Brain Sciences, 5(1), 134-141. https://psycnet.apa.org/doi/10.1177/2372732217749254

Smith, M. L., \& Seward, R. K. (2020). Introduction: Governing Openness in an Unequal World. In Making Open Development Inclusive: Lessons from IDRC Research (pp. 1-19). The MIT Press. https://idl-bnc-idrc.dspacedirect.org/bitstream/handle/10625/59418/IDL59418.pdf?sequence $=2 \&$ isAllowed $=y$

Smith, R. (2006). er review: A flawed process at the heart of science and journals. Journal of the Royal Society of Medicine, 99, 178-182. https://dx.doi.org/10.1258\%2Fjrsm.99.4.178

Snively, G., \& Williams, W. L. (2016). Chapter 1-Braiding Indigenous Science with Western Science. In Knowing home: Braiding Indigenous science with western science (Vol. 1, pp. 3-13). University of Victoria.

Solórzano, D. G., \& Yosso, T. J. (2002). Critical Race Methodology: Counter-Storytelling as an Analytical Framework for Education Research. Qualitative Inquiry, 8(1), 23-44. https://doi.org/10.1177/107780040200800103

Spies, J. R. (2013). The Open Science Framework: Improving Science by Making It Open and Accessible [Dissertation, University of Virginia]. https://doi.org/10.31237/osf.io/t23za

Spivak, G. C. (1990). The Post-Colonial Critic: Interviews, Strategies, Dialogues. Routledge. https://books.google.ca/books?hl=en\&lr=\&id=- 
CVpAwAAQBAJ\&oi=fnd\&pg=PP1\&ots=W9V5paEx2t\&sig=eoLpKz9mmzDSH1jSOK

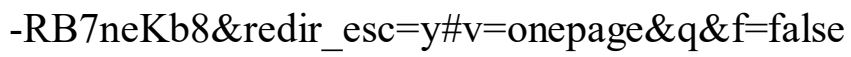

Sprott, W. (1954). Introduction. The Sociological Review, 2(1), 5-11. https://doi.org/10.1111\%2Fj.1467-954X.1954.tb03194.x

Standard. (n.d.). In Merriam Webster. https://www.merriam-webster.com/dictionary/standard Strauss, A., \& Corbin, J. (1990). Basics of qualitative research: Grounded theory procedures and techniques (First). Sage Publications, Inc.

Subedi, R., Greenberg, L., \& Martin, T. (2020). COVID-19 mortality rates in Canada's ethnocultural neighbourhoods. Statistics Canada. https://www150.statcan.gc.ca/n1/pub/45-280001/2020001/article/00079-eng.htm

Suber, P. (2009). Timeline of the open access movement. Harvard University Office for Scholarly Communication. https://dash.harvard.edu/handle/1/4724185.

Taylor, D. R. F. (2013). Developments in the theory and practice of cybercartography: Applications and indigenous mapping (Second, Vol. 4). Elsevier.

The First Nations Information Governance Centre. (2014). Ownership, Control, Access and Possession (OCAP $\left.{ }^{T M}\right)$ : The Path to First Nations Information Governance. The First Nations Information Governance Centre. https://fnigc.ca/wpcontent/uploads/2020/09/5776c4ee9387f966e6771aa93a04f389_ocap_path_to_fn_inform ation_governance_en_final.pdf

Turnbull, D. (2009). Introduction: Futures for Indigenous Knowledges. Futures, 41(1), 1-5.

UNESCO. (n.d.). What is Local and Indigenous Knowledge? UNESCO. http://www.unesco.org/new/en/natural-sciences/priority-areas/links/relatedinformation/what-is-local-and-indigenous-knowledge 
UNESCO. (2020). Towards a UNESCO Recommendation on Open Science: Building a Global Consensus on Open Science. UNESCO. https://en.unesco.org/sites/default/files/open_science_brochure_en.pdf

University of Lapland. (n.d.). Traditional Knowledge. Arctic Centre, University of Lapland. https://www.arcticcentre.org/EN/arcticregion/Arctic-Indigenous-Peoples/Traditionalknowledge

Valsiner, J. (2006). Review Essay: "Open Access" and its Social Context: New Colonialism in the Making? Forum: Qualitative Social Research, 7(2), 1-11. https://doi.org/10.17169/fqs-7.2.116

Veblen, T. (1908). On the Nature of Capital. Quarterly Journal of Economics (22)4, 517-542.

Verran, H., \& Turnbull, D. (1995). Chapter 6: Science and other indigenous knowledge systems. In Handbook of Science and Technology Studies, Revised Edition (pp. 115-139). SAGE Publications, Inc. http://dx.doi.org/10.4135/9781412990127

Viruru, R. (2006). Postcolonial Technologies of Power: Standardized Testing and Representing Diverse Young Children. International Journal of Educational Policy, Research \& Practice: Reconceptualizing Childhood Studies, 7, 49-70.

Wikipedia. (n.d.). Intellectual property. In Wikipedia. https://en.wikipedia.org/wiki/Intellectual_property

Wilkinson, M., Dumontier, M., Aalbersberg, I., \& Appleton, G. (2016). The FAIR Guiding Principles for scientific data management and stewardship. Scientific Data, 3(1). https://doi.org/10.1038/sdata.2016.18

Wilson, K. (2018). Pulling Together: Foundations Guide. BCcampus. https://opentextbc.ca/indigenizationfoundations/ 
Winner, L. (1977). Autonomous Technology: Technics-Out-of-Control as a Theme in Political Thought. MIT Press. https://philpapers.org/rec/WINATT

Zaveri, S. (2020). Gender and Equity in Openness: Forgotten Spaces. In Making Open

Development Inclusive: Lessons from IDRC Research (pp. 87-118). The MIT Press. 